\begin{tabular}{|c|l|}
\hline Title & CONTACT GEOMETRY OF SECOND ORDER I \\
\hline Author(s) & Yamaguchi, K. \\
\hline Citation & Hokkaido University Preprint Series in Mathematics, 927, 1-39 \\
\hline Issue Date & 2008-10-8 \\
\hline DOI & 10.14943/84421 \\
\hline Doc URL & http://hdl.handle.net/2115/70235 \\
\hline Type & bulletin (article) \\
\hline File Information & pre927.pdf \\
\hline
\end{tabular}

Instructions for use 


\title{
CONTACT GEOMETRY OF SECOND ORDER I
}

\author{
KEIZO YAMAGUCHI
}

\section{INTRODUCTION}

In [C1] and [C2], E.Cartan studied involutive systems of second order partial differential equations for a scalar function with 2 or 3 independent variables, following the tradition of the geometric theory of partial differential equations developed by Monge, Jacobi, Lie , Darboux, Goursat and others. In fact he investigated the contact equivalence and the integration problems of such involutive systems of second order. In this course, he found out the link between the contact equivalence of involutive systems of second order and the geometry of differential systems (Pffafian systems ) on five dimensional spaces.

The main purpose of the present paper is to reformulate his study as the Contact Geometry of Second Order. As is well known, the classical theory of systems of the first order partial differential equations for a scalar function can be rephrased as the submanifold theory of contact manifolds. In this spirit, we formulate the submanifold theory of second order contact manifols as the geometry of $P D$ manifolds of second order ([Y1], [Y6] see $\S 4)$.

By Bäcklund Theorem (see $§ 2.2$ ), the symbols of second order equations become the first invariants under contact transformations. In fact, in [C2], E.Cartan first classified involutive symbols algebraically and wrote the structure equations of such involutive systems of second order with 3 independent variables. To capture good classes of second order equations, we cannot pursue this line in general (see the discussion in §3.3). Our guiding principle in this paper is to utilize Parabolic Geometries, directly or combined with the reduction procedures, to find good classes of $P D$ manifolds of second order. Here the Parabolic Geometry is a geometry modeled after the homogeneous space $G / G^{\prime}$, where $G$ is a (semi-)simple Lie group and $G^{\prime}$ is a parabolic subgroup of $G$ (cf. [Bai]). Precisely, in this paper, we mean, by a Parabolic Geometry, the Geometry associated with the Simple Graded Lie Algebra in the sense of N.Tanaka ([T4]). As for the reduction procedures, we will establish the First Reduction Theorem for $P D$ manifolds admitting non-trivial Chauchy characteristic systems in $\S 4$ and will treat the second reduction procedures (two step reductions) as Part II in the sequel to this paper.

Now let us proceed to describe the contents of each sections. In $\S 2$, we will recall the geometric (Grassmannian) construction of Jet spaces and prepare basics of differential systems, especially the Tanaka Theory of (linear) differential systems. In $\S 3$, we will discuss the symbols of second order equations as the first invariants in the Contact Geometry of Second Order. We will formulate the submanifold theory of second order contact manifols in $\S 4$ as the geometry of $P D$ manifolds of second order and establish the First Reduction Theorem. In $\S 5$, we will first prepare the notation for the simple graded Lie algebras and state the Prolongation Theorem. Then we will exhibit Parabolic Geometries, which directly correspond to the geometry of $P D$ manifolds of second order. Finally, in $\S 6$, we will discuss one of the Typical classes of involutive systems of second order as the application of the First Reduction Theorem and will show several examples 
of Parabolic Geometries, which are linked to the geometry of $P D$ manifolds of second order through the First Reduction Theorem.

This paper constitutes the extended version of our previous paper [Y6].

\section{Geometry of Jet Spaces.}

We will recall the geometric construction of Jet spaces and fix our notations for the basic notion for differential systems, following [Y5] and [Y7].

2.1. Spaces of Contact Elements. Let us start with the construction of the space $J(M, n)$ of contact elements to $M$ : Let $M$ be a (real or complex) manifold of dimension $m+n$. Fixing the number $n$, we consider the space of $n$-dimensional contact elements to $M$, i.e., the Grassmannian bundle over $M$ consisting of all $n$-dimensional contact elements to $M$;

$$
J(M, n)=\bigcup_{x \in M} J_{x} \stackrel{\pi}{\longrightarrow} M,
$$

where $J_{x}=\operatorname{Gr}\left(T_{x}(M), n\right)$ is the Grassmann manifold of all $n$-dimensional subspaces of the tangent space $T_{x}(M)$ to $M$ at $x$. Each element $u \in J(M, n)$ is a linear subspace of $T_{x}(M)$ of codimension $m$, where $x=\pi(u)$. Hence we have a differential system $C$ of codimension $m$ on $J(M, n)$ by putting:

$$
C(u)=\pi_{*}^{-1}(u) \subset T_{u}(J(M, n)) \stackrel{\pi_{*}}{\longrightarrow} T_{x}(M) .
$$

for each $u \in J(M, n) . \quad C$ is called the Canonical System on $J(M, n)$. Introducing the inhomogeneous Grassmann coordinate $\left(x_{1}, \cdots, x_{n}, z^{1}, \cdots, z^{m}, p_{1}^{1}, \cdots, p_{n}^{m}\right)$ of $J(M, n)$ around $u_{o} \in J(M, n), C$ is defined by;

$$
C=\left\{\varpi^{1}=\cdots=\varpi^{m}=0\right\},
$$

where

$$
\varpi^{\alpha}=d z^{\alpha}-\sum_{i=1}^{n} p_{i}^{\alpha} d x_{i}, \quad(\alpha=1, \cdots, m) .
$$

Here $\left(x_{1}, \cdots, x_{n}, z^{1}, \cdots, z^{m}\right)$ is a coordinate system of $M$ around $x_{o}=\pi\left(u_{o}\right)$ such that $\left.d x_{1} \wedge \cdots \wedge d x_{n}\right|_{u_{o}} \neq 0$. Coordinate functions $p_{i}^{\alpha}$ are introduced by

$$
\left.d z^{\alpha}\right|_{u}=\left.\sum_{i=1}^{n} p_{i}^{\alpha}(u) d x_{i}\right|_{u} .
$$

$(J(M, n), C)$ is the (geometric) 1 -jet space for $n$-dimensional submanifolds in $M$. Let $M, \hat{M}$ be manifolds (of dimension $m+n$ ) and $\varphi: M \rightarrow \hat{M}$ be a diffeomorphism between them. Then $\varphi$ induces the isomorphism $\varphi_{*}:(J(M, n), C) \rightarrow(J(\hat{M}, n), \hat{C})$, i.e., the differential map $\varphi_{*}: J(M, n) \rightarrow J(\hat{M}, n)$ is a diffeomorphism sending $C$ onto $\hat{C}$.

2.2. Second Order Contact Manifolds. Let $J$ be a manifold and $C$ be a (linear) differential system on $J$ of codimension 1 . Namely $C$ is a subundle of $T(J)$ of codimension 1. Thus, locally at each point $u$ of $J$, there exists a 1 -form $\varpi$ defined around $u \in J$ such that

$$
C=\{\varpi=0\} .
$$

Then $(J, C)$ is called a contact manifold if $\varpi \wedge(d \varpi)^{n}$ forms a volume element of $J$. This condition is equivalent to the following conditions (1), (2) or (3);

(1) The restriction $\left.d \varpi\right|_{C}$ of $d \varpi$ to $C(u)$ is non-degenerate at each point $u \in J$. 
(2) There exists a coframe $\left\{\varpi, \omega_{1}, \ldots, \omega_{n}, \pi_{1}, \ldots, \pi_{n}\right\}$ defined around $u \in J$ such that the following holds;

$$
d \varpi \equiv \omega_{1} \wedge \pi_{1}+\cdots+\omega_{n} \wedge \pi_{n} \quad(\bmod \quad \varpi)
$$

(3) The Cauchy characteristic system $\mathrm{Ch}(C)$ of $C$ is trivial (see $\S 2.3$ below).

By the Darboux Theorem, a contact manifold $(J, C)$ of dimension $2 n+1$ can be regarded locally as a space of 1-jets for one unknown function. Namely, at each point of $(J, C)$, there exists a canonical coordinate system $\left(x_{1}, \ldots, x_{n}, z, p_{1}, \ldots, p_{n}\right)$ such that

$$
C=\left\{d z-\sum_{i=1}^{n} p_{i} d x_{i}=0\right\}
$$

Starting from a contact manifold $(J, C)$, we can construct the geometric second order jet space $(L(J), E)$ as follows: We consider the Lagrange-Grassmann bundle $L(J)$ over $J$ consisting of all $n$-dimensional integral elements of $(J, C)$;

$$
L(J)=\bigcup_{u \in J} L_{u} \stackrel{\pi}{\longrightarrow} J
$$

where $L_{u}$ is the Grassmann manifolds of all lagrangian (or legendrian) subspaces of the symplectic vector space $(C(u), d \varpi)$. Here $\varpi$ is a local contact form on $J$. Then the canonical system $E$ on $L(J)$ is defined by

$$
E(v)=\pi_{*}^{-1}(v) \subset T_{v}(L(J)) \stackrel{\pi_{*}}{\longrightarrow} T_{u}(J), \quad \text { for } \quad v \in L(J) .
$$

Let us fix a point $v_{o} \in L(J)$. Starting from a canonical coordinate system $\left(x_{1}, \cdots, x_{n}, z, p_{1}\right.$, $\left.\cdots, p_{n}\right)$ of $(J, C)$ around $u_{o}=\pi\left(v_{o}\right)$ such that $\left.d x_{1} \wedge \cdots \wedge d x_{n}\right|_{v_{o}} \neq 0$, we can introduce a coordinate system $\left(x_{i}, z, p_{i}, p_{i j}\right)(1 \leqq i \leqq j \leqq n)$ by defining coordinate functions $p_{i j}$ as follows;

$$
\left.d p_{i}\right|_{v}=\left.\sum_{i=1}^{n} p_{i j}(v) d x_{j}\right|_{v} .
$$

Then, since $v \in C(u)$, we have $\left.d z\right|_{v}=\left.\sum_{i=1}^{n} p_{i}(u) d x\right|_{v}$ and, since $\left.d \varpi\right|_{v}=0$, we get $p_{i j}=p_{j i}$.

Thus $E$ is defined on this canonical coordinate system by

$$
E=\left\{\varpi=\varpi_{1}=\cdots=\varpi_{n}=0\right\},
$$

where

$$
\varpi=d z-\sum_{i=1}^{n} p_{i} d x_{i}, \quad \text { and } \quad \varpi_{i}=d p_{i}-\sum_{j=1}^{n} p_{i j} d x_{j} \quad \text { for } \quad i=1, \cdots, n .
$$

Let $(J, C),(\hat{J}, \hat{C})$ be contact manifolds of dimension $2 n+1$ and $\varphi:(J, C) \rightarrow(\hat{J}, \hat{C})$ be a contact diffeomorphism between them. Then $\varphi$ induces an isomorphism $\varphi_{*}:(L(J), E) \rightarrow$ $(L(\hat{J}), \hat{E})$. Conversely we have (cf. Theorem $3.2[\mathrm{Y} 1])$

Theorem 2.1. (Bäcklund) Let $(J, C)$ and $(\hat{J}, \hat{C})$ be contact manifolds of dimension $2 n+1$. Then, for an isomorphism $\Phi:(L(J), E) \rightarrow(L(\hat{J}), \hat{E})$, there exists a contact diffeomorphism $\varphi:(J, C) \rightarrow(\hat{J}, \hat{C})$ such that $\Phi=\varphi_{*}$. 
2.3. Derived Systems and Cauchy Characteristic Systems. Now we prepare basic notions for (linear) differential systems (or Pfaffian systems). By a (linear) differential system $(M, D)$, we mean a subbundle $D$ of the tangent bundle $T(M)$ of a manifold $M$ of dimension $d$. Locally $D$ is defined by 1 -forms $\omega_{1}, \ldots, \omega_{d-r}$ such that $\omega_{1} \wedge \cdots \wedge \omega_{d-r} \neq 0$ at each point, where $r$ is the rank of $D$;

$$
D=\left\{\omega_{1}=\cdots=\omega_{d-r}=0\right\} .
$$

For two differential systems $(M, D)$ and $(\hat{M}, \hat{D})$, a diffeomorphism $\varphi$ of $M$ onto $\hat{M}$ is called an isomorphism of $(M, D)$ onto $(\hat{M}, \hat{D})$ if the differential map $\varphi_{*}$ of $\varphi$ sends $D$ onto $\hat{D}$.

For a non-integrable differential system $D$, we consider the Derived System $\partial D$ of $D$, which is defined, in terms of sections, by

$$
\partial \mathcal{D}=\mathcal{D}+[\mathcal{D}, \mathcal{D}]
$$

where $\mathcal{D}=\Gamma(D)$ denotes the space of sections of $D$.

Furthermore the Cauchy Characteristic System Ch $(D)$ of $(M, D)$ is defined at each point $x \in M$ by

$$
\left.\operatorname{Ch}(D)(x)=\{X \in D(x) \mid X\rfloor d \omega_{i} \equiv 0 \quad\left(\bmod \omega_{1}, \ldots, \omega_{s}\right) \quad \text { for } i=1, \ldots, s\right\},
$$

where $\rfloor$ denotes the interior multiplication, i.e., $X\rfloor d \omega(Y)=d \omega(X, Y)$ and $s=d-r$. When $\mathrm{Ch}(D)$ is a differential system (i.e., has constant rank), it is always completely integrable.

Moreover Higher Derived Systems $\partial^{k} D$ are usually defined successively (cf. $\left[\mathrm{BCG}_{3}\right]$ ) by

$$
\partial^{k} D=\partial\left(\partial^{k-1} D\right)
$$

where we put $\partial^{0} D=D$ for convention.

On the other hand we define the $k$-th Weak Derived System $\partial^{(k)} D$ of $D$ inductively by

$$
\partial^{(k)} \mathcal{D}=\partial^{(k-1)} \mathcal{D}+\left[\mathcal{D}, \partial^{(k-1)} \mathcal{D}\right]
$$

where $\partial^{(0)} D=D$ and $\partial^{(k)} \mathcal{D}$ denotes the space of sections of $\partial^{(k)} D$.

2.4. Review of Tanaka Theory. A differential system $(M, D)$ is called regular, if $D^{-(k+1)}=\partial^{(k)} D$ are subbundles of $T(M)$ for every integer $k \geqq 1$. For a regular differential system $(M, D)$, we have ( [T2], Proposition 1.1)

(S1) There exists a unique integer $\mu>0$ such that, for all $k \geqq \mu$,

$$
D^{-k}=\cdots=D^{-\mu} \supsetneqq D^{-\mu+1} \supsetneqq \cdots \supsetneqq D^{-2} \supsetneqq D^{-1}=D,
$$

where $\mathcal{D}^{p}$ denotes the space of sections of $D^{p}$. (S2) implies subbundles $D^{p}$ define a filtration on $M$.

Let $(M, D)$ be a regular differential system such that $T(M)=D^{-\mu}$. As a first invariant for non-integrable differential systems, we now define the symbol algebra $\mathfrak{m}(x)$ associated with a differential system $(M, D)$ at $x \in M$, which was introduced by N. Tanaka [T2].

We put $\mathfrak{g}_{-1}(x)=D^{-1}(x), \mathfrak{g}_{p}(x)=D^{p}(x) / D^{p+1}(x)(p<-1)$ and

$$
\mathfrak{m}(x)=\bigoplus_{p=-1}^{-\mu} \mathfrak{g}_{p}(x)
$$


Let $\pi_{p}$ be the projection of $D^{p}(x)$ onto $\mathfrak{g}_{p}(x)$. Then, for $X \in \mathfrak{g}_{p}(x)$ and $Y \in \mathfrak{g}_{q}(x)$, the bracket product $[X, Y] \in \mathfrak{g}_{p+q}(x)$ is defined by

$$
[X, Y]=\pi_{p+q}\left([\tilde{X}, \tilde{Y}]_{x}\right)
$$

where $\tilde{X}$ and $\tilde{Y}$ are any element of $\mathcal{D}^{p}$ and $\mathcal{D}^{q}$ respectively such that $\pi_{p}\left(\tilde{X}_{x}\right)=X$ and $\pi_{q}\left(\tilde{Y}_{x}\right)=Y$.

Endowed with this bracket operation, by $(S 2)$ above, $\mathfrak{m}(x)$ becomes a nilpotent graded Lie algebra such that $\operatorname{dim} \mathfrak{m}(x)=\operatorname{dim} M$ and satisfies

$$
\mathfrak{g}_{p}(x)=\left[\mathfrak{g}_{p+1}(x), \mathfrak{g}_{-1}(x)\right] \quad \text { for } p<-1 .
$$

We call $\mathfrak{m}(x)$ the Symbol Algebra of $(M, D)$ at $x \in M$.

Furthermore, let $\mathfrak{m}$ be a FGLA (fundamental graded Lie algebra) of $\mu$-th kind, that is,

$$
\mathfrak{m}=\bigoplus_{p=-1}^{-\mu} \mathfrak{g}_{p}
$$

is a nilpotent graded Lie algebra such that

$$
\mathfrak{g}_{p}=\left[\mathfrak{g}_{p+1}, \mathfrak{g}_{-1}\right] \quad \text { for } p<-1 .
$$

Then $(M, D)$ is called of type $\mathfrak{m}$ if the symbol algebra $\mathfrak{m}(x)$ is isomorphic to $\mathfrak{m}$ at each $x \in M$.

Conversely, given a FGLA $\mathfrak{m}=\bigoplus_{p=-1}^{-\mu} \mathfrak{g}_{p}$, we can construct a model differential system of type $\mathfrak{m}$ as follows: Let $M(\mathfrak{m})$ be the simply connected Lie group with Lie algebra $\mathfrak{m}$. Identifying $\mathfrak{m}$ with the Lie algebra of left invariant vector fields on $M(\mathfrak{m}), \mathfrak{g}_{-1}$ defines a left invariant subbundle $D_{\mathfrak{m}}$ of $T(M(\mathfrak{m}))$. By definition of symbol algebras, it is easy to see that $\left(M(\mathfrak{m}), D_{\mathfrak{m}}\right)$ is a regular differential system of type $\mathfrak{m} .\left(M(\mathfrak{m}), D_{\mathfrak{m}}\right)$ is called the Standard Differential System of Type $\mathfrak{m}$. The Lie algebra of all infinitesimal automorphisms of $\left(M(\mathfrak{m}), D_{\mathfrak{m}}\right)$ can be calculated algebraically as the Prolongation $\mathfrak{g}(\mathfrak{m})$ of $\mathfrak{m}([\mathrm{T} 1]$, cf. $[\mathrm{Y} 5])$.

In fact, let $\mathfrak{m}=\bigoplus_{p<0} \mathfrak{g}_{p}$ be a fundamental graded Lie algebra of $\mu$-th kind defined over a field $\mathbb{K}$. Here $\mathbb{K}$ denotes the field of real numbers $\mathbb{R}$ or that of complex numbers $\mathbb{C}$. We put

$$
\mathfrak{g}(\mathfrak{m})=\bigoplus_{p \in \mathbb{Z}} \mathfrak{g}_{p}(\mathfrak{m})
$$

where $\mathfrak{g}_{p}(\mathfrak{m})=\mathfrak{g}_{p}$ for $p<0, \mathfrak{g}_{0}(\mathfrak{m})$ is the Lie algebra of all (gradation preserving) derivations of graded Lie algebra $\mathfrak{m}$ and $\mathfrak{g}_{k}(\mathfrak{m})$ is defined inductively by the following for $k \geqq 1$;

$$
\mathfrak{g}_{k}(\mathfrak{m})=\left\{u \in \bigoplus_{p<0} \mathfrak{g}_{p+k} \otimes \mathfrak{g}_{p}^{*} \mid u([Y, Z])=[u(Y), Z]-[u(Z), Y]\right\} .
$$

Thus, as a vector space over $\mathbb{K}, \mathfrak{g}_{k}(\mathfrak{m})$ is a linear subspace of End $\left(\mathfrak{m}, \mathfrak{m}^{k}\right)=\mathfrak{m}^{k} \otimes \mathfrak{m}^{*}$, where $\mathfrak{m}^{k}=\mathfrak{m} \oplus \mathfrak{g}_{0}(\mathfrak{m}) \oplus \cdots \oplus \mathfrak{g}_{k-1}(\mathfrak{m})$. The bracket operation of $\mathfrak{g}(\mathfrak{m})$ is given accordingly (see [T1], [Y5] for detail).

The structure of the Lie algebra $\mathcal{A}\left(M(\mathfrak{m}), D_{\mathfrak{m}}\right)$ of all infinitesimal automorphisms of $\left(M(\mathfrak{m}), D_{\mathfrak{m}}\right)$ can be described by $\mathfrak{g}(\mathfrak{m})$. Especially $\mathcal{A}\left(M(\mathfrak{m}), D_{\mathfrak{m}}\right)$ is isomorphic to $\mathfrak{g}(\mathfrak{m})$, when $\mathfrak{g}(\mathfrak{m})$ is finite dimensional. by

Let $\mathfrak{g}_{0}$ be a subalgebra of $\mathfrak{g}_{0}(\mathfrak{m})$. We define a subspace $\mathfrak{g}_{k}$ of $\mathfrak{g}_{k}(\mathfrak{m})$ for $k \geqq 1$ inductively

$$
\mathfrak{g}_{k}=\left\{u \in \mathfrak{g}_{k}(\mathfrak{m}) \mid\left[u, \mathfrak{g}_{-1}\right] \subset \mathfrak{g}_{k-1}\right\}
$$


Then, putting

$$
\mathfrak{g}\left(\mathfrak{m}, \mathfrak{g}_{0}\right)=\mathfrak{m} \oplus \bigoplus_{k \geqq 0} \mathfrak{g}_{k}
$$

we see, with the generating condition of $\mathfrak{m}$, that $\mathfrak{g}\left(\mathfrak{m}, \mathfrak{g}_{0}\right)$ is a graded subalgebra of $\mathfrak{g}(\mathfrak{m})$. $\mathfrak{g}\left(\mathfrak{m}, \mathfrak{g}_{0}\right)$ is called the prolongation of $\left(\mathfrak{m}, \mathfrak{g}_{0}\right)$.

We will recall in $\S 5.1$ when $\mathfrak{g}(\mathfrak{m})$ or $\mathfrak{g}\left(\mathfrak{m}, \mathfrak{g}_{0}\right)$ becomes finite dimensional and simple.

2.5. Symbol Algebra of $(L(J), E)$. As an example to calculate symbol algebras, let us show that $(L(J), E)$ is a regular differential system of type $\mathfrak{c}^{2}(n)$ :

$$
\mathfrak{c}^{2}(n)=\mathfrak{c}_{-3} \oplus \mathfrak{c}_{-2} \oplus \mathfrak{c}_{-1},
$$

where $\mathfrak{c}_{-3}=\mathbb{R}, \mathfrak{c}_{-2}=V^{*}$ and $\mathfrak{c}_{-1}=V \oplus S^{2}\left(V^{*}\right)$. Here $V$ is a vector space of dimension $n$ and the bracket product of $\mathfrak{c}^{2}(n)$ is defined accordingly through the pairing between $V$ and $V^{*}$ such that $V$ and $S^{2}\left(V^{*}\right)$ are both abelian subspaces of $\mathfrak{c}_{-1}$. This fact can be checked as follows: Let us take a canonical coordinate system $\left(x_{i}, z, p_{i}, p_{i j}\right)(1 \leqq i \leqq j \leqq n)$ of $(L(J), E)$. Then we have a coframe $\left\{\varpi, \varpi_{i}, d x_{i}, d p_{i j}\right\}(1 \leqq i \leqq j \leqq n)$ at each point in this coordinate neiborhood, where $\varpi=d z-\sum_{i=1}^{n} p_{i} d x_{i}, \varpi_{i}=d p_{i}-\sum_{j=1}^{n} p_{i j} d x_{j}$ $(i=1, \cdots, n)$. Now take the dual frame $\left\{\frac{\partial}{\partial z}, \frac{\partial}{\partial p_{i}}, \frac{d}{d x_{i}}, \frac{\partial}{\partial p_{i j}}\right\}$, of this coframe, where

$$
\frac{d}{d x_{i}}=\frac{\partial}{\partial x_{i}}+p_{i} \frac{\partial}{\partial z}+\sum_{j=1}^{n} p_{i j} \frac{\partial}{\partial p_{j}}
$$

is the classical notation. Notice that $\left\{\frac{d}{d x_{i}}, \frac{\partial}{\partial p_{i j}}\right\}(1 \leqq i \leqq j \leqq n)$ forms a free basis of $\Gamma(E)$. Then we have

$$
\begin{gathered}
{\left[\frac{\partial}{\partial p_{i i}}, \frac{d}{d x_{j}}\right]=\delta_{j}^{i} \frac{\partial}{\partial p_{i}}, \quad\left[\frac{\partial}{\partial p_{i j}}, \frac{d}{d x_{k}}\right]=\delta_{k}^{i} \frac{\partial}{\partial p_{i}}+\delta_{k}^{j} \frac{\partial}{\partial p_{j}} \quad \text { for } i \neq j,} \\
{\left[\frac{\partial}{\partial p_{i}}, \frac{d}{d x_{j}}\right]=\delta_{j}^{i} \frac{\partial}{\partial z}, \quad\left[\frac{d}{d x_{i}}, \frac{d}{d x_{j}}\right]=0 .}
\end{gathered}
$$

It follows that $T(L(J))=\partial^{(2)} E$ and the derived system $\partial E$ of $E$ satisfies the following :

$$
\partial E=\{\varpi=0\}=\pi_{*}^{-1} C, \quad \operatorname{Ch}(\partial E)=\operatorname{Ker} \pi_{*} .
$$

These facts provide the proof of Theorem 1 (cf. Theorem 3.2 [Y1]).

Moreover, in terms of the defining 1-forms of $E$ and $\partial E$ around $v \in L(J)$, the structure of the symbol algebra $\mathfrak{c}^{2}(n)$ can be described by the following Structure Equation of E.Cartan $([\mathrm{C} 1],[\mathrm{C} 2])$;

$$
\begin{aligned}
& d \varpi \equiv \omega_{1} \wedge \varpi_{1}+\cdots+\omega_{n} \wedge \varpi_{n} \quad\left(\bmod \quad \varpi, \varpi_{i} \wedge \varpi_{j}(1 \leqq i \leqq j \leqq n)\right) \\
& \left\{\begin{aligned}
d \varpi_{1} & \equiv \omega_{1} \wedge \pi_{11}+\cdots+\omega_{n} \wedge \pi_{1 n} \\
& \ldots \\
d \varpi_{n} & \equiv \omega_{1} \wedge \pi_{n 1}+\cdots+\omega_{n} \wedge \pi_{n n}
\end{aligned}\left(\bmod \quad \varpi, \varpi_{1}, \ldots, \varpi_{n}\right)\right.
\end{aligned}
$$

where $\partial E=\{\varpi=0\}, E=\left\{\varpi=\varpi_{1}=\cdots=\varpi_{n}=0\right\}$ and $\left\{\varpi, \varpi_{i}, \omega_{i}, \pi_{i j} \quad(1 \leqq i \leqq j \leqq\right.$ $n)\}$ forms a coframe around $v \in L(J)$. Here we understand that $\pi_{i j}=\pi_{j i}$.

Similarly we see that $(J(M, n), C)$ is a regular differential system of type $\mathfrak{c}^{1}(n, m)$ :

$$
\mathfrak{c}^{1}(n, m)=\mathfrak{c}_{-2} \oplus \mathfrak{c}_{-1},
$$

where $\mathfrak{c}_{-2}=W$ and $\mathfrak{c}_{-1}=V \oplus W \otimes V^{*}$ for vector spaces $V$ and $W$ of dimension $n$ and $m$ respectively, and the bracket product of $\mathfrak{c}^{1}(n, m)$ is defined accordingly through the pairing between $V$ and $V^{*}$ such that $V$ and $W \otimes V^{*}$ are both abelian subspaces of $\mathfrak{c}_{-1}$. 


\section{Symbols of Second Order Equations.}

In view of the Bäcklund Theorem, we will discuss the symbols of second order equations as the first invariants in the Contact Geometry of Second Order.

3.1. Symbol Algebras. Let $R$ be a submanifold of $L(J)$ satisfying the following condition:

$$
(R .0) \quad p: R \rightarrow J ; \text { submersion, }
$$

where $p=\left.\pi\right|_{R}$ and $\pi: L(J) \rightarrow J$ is the projection. This condition implies that the system of equations $R$ of second order contains no equations of first order. We have two differential systems $C^{1}=\partial E$ and $C^{2}=E$ on $L(J)$. We denote by $D^{1}$ and $D^{2}$ those differential systems on $R$ obtained by restricting $C^{1}$ and $C^{2}$ to $R$. Moreover we denote by the same symbols those 1 -forms obtained by restricting the defining 1 -forms $\left\{\varpi, \varpi_{1}, \cdots, \varpi_{n}\right\}$ of the canonical system $E$ to $R$, where $\varpi=d z-\sum_{i=1}^{n} p_{i} d x_{i}$, and $\varpi_{i}=d p_{i}-\sum_{j=1}^{n} p_{i j} d x_{j}$ $(i=1, \ldots, n)$. Then it follows from $(R .0)$ that these 1 -forms are independent at each point on $R$ and that

$$
D^{1}=\{\varpi=0\}, \quad D^{2}=\left\{\varpi=\varpi_{1}=\cdots=\varpi_{n}=0\right\} .
$$

Thus $D^{1}$ and $D^{2}$ are subbundles of $T(R)$ such that $\partial D^{2} \subset D^{1}$. Hence subbundles $D^{2}, D^{1}$ and $T(R)$ define a filtration on $R$. Namely, putting $D^{-1}=D^{2}, D^{-2}=D^{1}, D^{p}=T(R)$ for $p \leqq-3$, we have

$$
\left[\mathcal{D}^{p}, \mathcal{D}^{q}\right] \subset \mathcal{D}^{p+q} \quad \text { for } \quad p, q<0
$$

where $\mathcal{D}^{p}=\Gamma\left(D^{p}\right)$.

Now we define the Symbol Algebra $\mathfrak{s}(v)$ of $R$ at $v \in R$ by

$$
\mathfrak{s}(v)=\mathfrak{s}_{-3}(v) \oplus \mathfrak{s}_{-2}(v) \oplus \mathfrak{s}_{-1}(v),
$$

where $\mathfrak{s}_{-3}(v)=T_{v}(R) / D^{1}(v), \mathfrak{s}_{-2}(v)=D^{1}(v) / D^{2}(v)$ and $\mathfrak{s}_{-1}(v)=D^{2}(v)$. The bracket operation in $\mathfrak{s}(v)$ is defined, similarly as in $\S 2.3$, as follows: For $X \in \mathfrak{s}_{p}(v)$ and $Y \in \mathfrak{s}_{q}(v)$, let us take $\tilde{X} \in \mathcal{D}^{p}$ and $\tilde{Y} \in \mathcal{D}^{q}$ such that $X=\pi_{p}\left((\tilde{X})_{v}\right)$ and $Y=\pi_{q}\left((\tilde{Y})_{v}\right)$, where $\pi_{p}: D^{p}(v) \rightarrow \mathfrak{s}_{p}(v)$ is the projection. Then the bracket product is defined by

$$
[X, Y]=\pi_{p+q}\left([\tilde{X}, \tilde{Y}]_{v}\right) \in \mathfrak{s}_{p+q}(v) .
$$

The bracket product $[X, Y]$ is well-defined for $X \in \mathfrak{s}_{p}(v)$ and $Y \in \mathfrak{s}_{q}(v)$, i.e., is independent of the choice of $\tilde{X}$ and $\tilde{Y}$. In fact, in our case, this can be shown as follows: The defining $1-$ forms $\varpi, \varpi_{1}, \ldots, \varpi_{n}$ for $D^{1}$ and $D^{2}$ actually define a basis $\{A\}$ of $\mathfrak{s}_{-3}(v)$ and $\left\{B_{1}, \ldots, B_{n}\right\}$ of $\mathfrak{s}_{-2}(v)$ such that $\varpi(\tilde{A})=1, \pi_{-3}(\tilde{A})=A, \varpi_{i}\left(\tilde{B}_{j}\right)=\delta_{j}^{i}, \pi_{-2}\left(\tilde{B}_{i}\right)=B_{i}$ and $\tilde{B}_{i} \in D^{1}(v)$. Then, for $X_{1}, X_{2} \in \mathfrak{s}_{-1}(v)=D^{2}(v)$, we calculate

$$
d \varpi_{i}\left(X_{1}, X_{2}\right)=\tilde{X}_{1}\left(\varpi_{i}\left(\tilde{X}_{2}\right)\right)-\tilde{X}_{2}\left(\varpi_{i}\left(\tilde{X}_{1}\right)\right)-\varpi_{i}\left(\left[\tilde{X}_{1}, \tilde{X}_{2}\right]\right)=-\varpi_{i}\left(\left[\tilde{X}_{1}, \tilde{X}_{2}\right]\right) .
$$

Thus, putting $\beta_{i}=-d \varpi_{i}\left(X_{1}, X_{2}\right)$, we get

$$
\left[X_{1}, X_{2}\right]=\beta_{1} B_{1}+\cdots+\beta_{n} B_{n} \in \mathfrak{s}_{-2}(v) .
$$

For $X \in \mathfrak{s}_{-1}(v)$ and $Y \in \mathfrak{s}_{-2}(v)$, we calculate

$$
d \varpi\left(X, \tilde{Y}_{v}\right)=\tilde{X}_{v}(\varpi(\tilde{Y}))-\tilde{Y}_{v}(\varpi(\tilde{X}))-\varpi\left([\tilde{X}, \tilde{Y}]_{v}\right)=-\varpi\left([\tilde{X}, \tilde{Y}]_{v}\right) .
$$

Similarly we have $d \varpi\left(X_{1}, X_{2}\right)=0$ for $X_{1}, X_{2} \in \mathfrak{s}_{-1}(v)$. Thus $d \varpi\left(X, \tilde{Y}_{v}\right)$ depends only on $X \in \mathfrak{s}_{-1}(v)$ and $Y \in \mathfrak{s}_{-2}(v)$. Hence, putting $\alpha=-d \varpi\left(X, \tilde{Y}_{v}\right)$, we have

$$
[X, Y]=\alpha A \in \mathfrak{s}_{-3}(v) \text {. }
$$


Moreover it follows that, for $X \in \mathfrak{s}_{-1}(v)$,

$$
X\rfloor d \varpi(Y)=0 \quad \text { for } \forall Y \in D^{1}(v) \text { if and only if }\left[X, \mathfrak{s}_{-2}(v)\right]=0 .
$$

Hence, from $\operatorname{Ch}\left(D^{1}\right)=\operatorname{Ker} p_{*} \subset D^{2}$, we have, putting $\mathfrak{f}(v)=\operatorname{Ch}\left(D^{1}\right)(v)$,

$$
\mathfrak{f}(v)=\left\{X \in \mathfrak{s}_{-1}(v) \mid\left[X, \mathfrak{s}_{-2}(v)\right]=0\right\} .
$$

$\mathfrak{f}(v)$ is a subspace of $\mathfrak{s}_{-1}(v)$ of codimension $n$.

By the description of the bracket operation in $\mathfrak{s}(v)$ above, since $\varpi$ and $\varpi_{1}, \ldots, \varpi_{n}$ are the restriction of defining 1-forms of $C^{1}=\partial E$ and $C^{2}=E$ on $L(J)$, we immediately see that $\varpi$ and $\varpi_{1}, \ldots, \varpi_{n}$, at the same time, define bases of $\mathfrak{g}_{-3}(v)$ and $\mathfrak{g}_{-2}(v)$ of the symbol algebra $\mathfrak{m}(v)=\mathfrak{g}_{-3}(v) \oplus \mathfrak{g}_{-2}(v) \oplus \mathfrak{g}_{-1}(v)\left(\cong \mathfrak{c}^{2}(n)\right)$ of $(L(J), E)$ at $v \in L(J)$ so that $\mathfrak{s}(v)$ is a graded subalgebra of $\mathfrak{m}(v)$ satisfying $\mathfrak{s}_{-3}(v)=\mathfrak{g}_{-3}(v), \mathfrak{s}_{-2}(v)=\mathfrak{g}_{-2}(v)$ and $\mathfrak{f}(v)=T_{v}(R) \cap \mathrm{Ch}\left(C^{1}\right)(v)$.

Now we consider the following compatibility condition for $R$ :

$$
\text { (C) } \quad p^{(1)}: R^{(1)} \rightarrow R \text { is onto. }
$$

where $R^{(1)}$ is the first prolongation of $R$. Namely we assume that there exists an $n$ dimensional integral element $V$ of $\left(R, D^{2}\right)$ at each $v \in R$ such that

$$
\mathfrak{s}_{-1}(v)=V \oplus \mathfrak{f}(v) .
$$

$V$ is an abelian subalgebra in $\mathfrak{s}(v)$. By fixing a basis of $\mathfrak{s}_{-3}(v), \mathfrak{s}_{-3}(v)$ is identified with $\mathbb{R}$ and, through [,] : $\mathfrak{s}_{-2}(v) \times \mathfrak{s}_{-1}(v) \rightarrow \mathfrak{s}_{-3}(v) \cong \mathbb{R}, \mathfrak{s}_{-2}(v)$ is identified with $V^{*}$, since $V \cap \mathfrak{f}(v)=\{0\}$ and $\mathfrak{f}(v)=\left\{X \in \mathfrak{s}_{-1}(v) \mid\left[X, \mathfrak{s}_{-2}(v)\right]=0\right\}$. Moreover we have a map $\mu: \mathfrak{f}(v) \rightarrow S^{2}\left(V^{*}\right)$ defined by

$$
\mu(f)\left(v_{1}, v_{2}\right)=\left[\left[f, v_{1}\right], v_{2}\right] \in \mathfrak{s}_{-3}(v) \cong \mathbb{R} \quad \text { for } f \in \mathfrak{f}(v) .
$$

Here $\mu(f)\left(v_{1}, v_{2}\right)=\mu(f)\left(v_{2}, v_{1}\right)$ follows from $\left[v_{1}, v_{2}\right]=0$ and the Jacobi identity of $\mathfrak{s}(v)$. We can check the injectivity of $\mu$ as follows: If $\mu(f)=0$, we have $\left[f, v_{1}\right]=0$ for $\forall v_{1} \in V$ by $\mathfrak{s}_{-2}(v) \cong V^{*}$. Then we have $\left[f, \mathfrak{s}_{-1}(v)\right]=0$, since $\mathfrak{f}(v)$ is abelian, which implies $f \in \mathfrak{f}(v) \cap \operatorname{Ch}\left(D^{2}\right)(v)$. From $\operatorname{Ch}\left(D^{1}\right)(v) \cap \operatorname{Ch}\left(D^{2}\right)(v)=0$ (see $\S 4.1$ ), we obtain $f=0$.

Hence, by fixing a basis of $\mathfrak{s}_{-3}(v)$ and the brackets in $\mathfrak{s}(v)$, we obtain

$$
\mathfrak{s}_{-3}(v) \cong \mathbb{R}, \quad \mathfrak{s}_{-2}(v) \cong V^{*}, \quad \mathfrak{s}_{-1}(v)=V \oplus \mathfrak{f}(v) \quad \text { and } \quad \mathfrak{f}(v) \subset S^{2}\left(V^{*}\right) .
$$

Thus $\mathfrak{f}(v) \subset S^{2}\left(V^{*}\right)$ is the first invariant of $R$ under contact transformation. We will first examine $\mathfrak{f}(v) \subset S^{2}\left(V^{*}\right)$ in the case $\operatorname{dim} V=2$ in the next section.

3.2. Case $n=2$. When $\operatorname{dim} V=2$, we have $\operatorname{dim} S^{2}\left(V^{*}\right)=3$. Through the natural pairing of $S^{2}(V)$ and $S^{2}\left(V^{*}\right)$ as subspaces of $V \otimes V$ and $V^{*} \otimes V^{*}$, we identify $S^{2}(V)$ with the dual space of $S^{2}\left(V^{*}\right)$. For a basis $\left\{e_{1}, e_{2}\right\}$ of $V$, we have a basis $\left\{e_{1}^{*} \odot e_{1}^{*}, 2 e_{1}^{*} \odot e_{2}^{*}, e_{2}^{*} \odot e_{2}^{*}\right\}$ of $S^{2}\left(V^{*}\right)$ and its dual basis $\left\{e_{1} \odot e_{1}, e_{1} \odot e_{2}, e_{2} \odot e_{2}\right\}$ of $S^{2}(V)$, where $\left\{e_{1}^{*}, e_{2}^{*}\right\}$ is the dual basis of $\left\{e_{1} . e_{2}\right\}$ and $e_{i} \odot e_{j}=\frac{1}{2}\left(e_{i} \otimes e_{j}+e_{j} \otimes e_{i}\right)$. For a subspace $\mathfrak{f}$ of $S^{2}\left(V^{*}\right)$, we denote by $\mathfrak{f}^{\perp}$ the annihilator of $\mathfrak{f}$ in $S^{2}(V)$.

Now let us classify subspaces $\mathfrak{f}$ of $S^{2}\left(V^{*}\right)$ under the action of $G L(V)$.

(1) $\operatorname{codim} \mathfrak{f}=1$

In this case $\operatorname{dim} \mathfrak{f}^{\perp}=1$. Hence we can classify a generator $f$ of $\mathfrak{f}^{\perp}$ as a quadratic form and obtain the following classification into three cases, i.e., there exists a basis of $V$ such that

$$
\mathfrak{f}^{\perp}=\left\{\begin{array}{l}
\left\langle\left\{e_{1} \odot e_{1}\right\}\right\rangle, \\
\left\langle\left\{e_{1} \odot e_{2}\right\}\right\rangle, \\
\left(\left\langle\left\{e_{1} \odot e_{1}+e_{2} \odot e_{2}\right\}\right\rangle\right)
\end{array}\right.
$$


The third case occurs when we classify over $\mathbb{R}$. Here $f$ is of rank 1 , rank 2 (indefinite) and rank 2 (definite) respectively.

(2) $\operatorname{codim} \mathfrak{f}=2$

In this case $\operatorname{dim} \mathfrak{f}=1$. Hence, similarly as above, we have the following classificasion into three cases, i.e., there exists a basis of $V$ such that

$$
\mathfrak{f}=\left\{\begin{array}{l}
\left\langle\left\{e_{2}^{*} \odot e_{2}^{*}\right\}\right\rangle, \\
\left\langle\left\{e_{1}^{*} \odot e_{2}^{*}\right\}\right\rangle, \\
\left(\left\langle\left\{e_{1}^{*} \odot e_{1}^{*}+e_{2}^{*} \odot e_{2}^{*}\right\}\right\rangle\right),
\end{array}\right.
$$

Thus, dually, we have

$$
\mathfrak{f}^{\perp}=\left\{\begin{array}{l}
\left\langle\left\{e_{1} \odot e_{1}, e_{1} \odot e_{2}\right\}\right\rangle, \\
\left\langle\left\{e_{1} \odot e_{1}, e_{2} \odot e_{2}\right\}\right\rangle, \\
\left(\left\langle\left\{e_{1} \odot e_{2}, e_{1} \odot e_{1}-e_{2} \odot e_{2}\right\}\right\rangle\right)
\end{array}\right.
$$

The third case occurs when we classify over $\mathbb{R}$. We note here that, for the prolongation $\mathfrak{f}^{(1)}=\mathfrak{f} \otimes V^{*} \cap S^{3}\left(V^{*}\right)$, we have $\left(\mathfrak{f}^{(1)}\right)^{\perp}=\left\langle\left\{e_{1} \odot e_{1} \odot e_{1}, e_{1} \odot e_{1} \odot e_{2}, e_{1} \odot\right.\right.$ $\left.\left.e_{2} \odot e_{2}, e_{2} \odot e_{2} \odot e_{2}\right\}\right\rangle$ for the second and third cases (see $\left.\S 3.3\right)$. Namely $\mathfrak{f}^{(1)}=\{0\}$ for the second and third cases, whereas the first case is involutive.

We can classify the symbol algebra $\mathfrak{s}(v)$ of $R$ at $v \in R$ according to the above classification for $\mathfrak{f}(v) \subset S^{2}\left(V^{*}\right)$ under the condition $(C)$. In the case codim $R=1, R$ is called parabolic, hyperbolic and elliptic at $v$ according as $f$ is of rank 1 , rank 2 (indefinite) and rank 2 (definite) respectively, where $f$ is a generator of $(\mathfrak{f}(v))^{\perp} \subset S^{2}(V)$ (see $\S 3.3$ ).

Now we assume the regularity for the symbol algebras. Namely assume that symbol algebras $\mathfrak{s}(v)$ of $R$ are locally isomorphic to the fixed symbol $\mathfrak{s}=\mathfrak{s}_{-3} \oplus \mathfrak{s}_{-2} \oplus \mathfrak{s}_{-1}$ where $\mathfrak{s}_{-3}=\mathbb{R}, \mathfrak{s}_{-2}=V^{*}$ and $\mathfrak{s}_{-1}=V \oplus \mathfrak{f}$ for the fixed $\mathfrak{f} \subset S^{2}\left(V^{*}\right)$. Then, for example, the Structure Equation reads as follows:

(i) $\mathfrak{f}^{\perp}=\left\langle\left\{e_{1} \odot e_{2}\right\}\right\rangle$

$$
\begin{cases}d \varpi & \equiv \omega_{1} \wedge \varpi_{1}+\omega_{2} \wedge \varpi_{2} \quad(\bmod \varpi) \\ d \varpi_{1} \equiv \omega_{1} \wedge \pi_{11} & \left(\bmod \quad \varpi, \varpi_{1}, \varpi_{2}\right) \\ d \varpi_{2} \equiv \quad \omega_{2} \wedge \pi_{22} & \left(\bmod \quad \varpi, \varpi_{1}, \varpi_{2}\right)\end{cases}
$$

(ii) $\mathfrak{f}^{\perp}=\left\langle\left\{e_{1} \odot e_{1}, e_{1} \odot e_{2}\right\}\right\rangle$

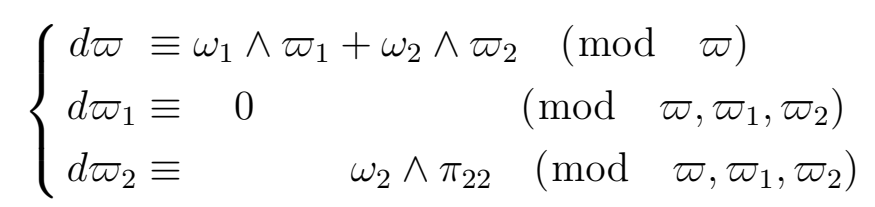

(iii) $\mathfrak{f}^{\perp}=\left\langle\left\{e_{1} \odot e_{1}, e_{2} \odot e_{2}\right\}\right\rangle$

$$
\left\{\begin{array}{l}
d \varpi \equiv \omega_{1} \wedge \varpi_{1}+\omega_{2} \wedge \varpi_{2} \quad(\bmod \varpi) \\
d \varpi_{1} \equiv \quad \omega_{2} \wedge \pi_{12} \quad\left(\bmod \quad \varpi, \varpi_{1}, \varpi_{2}\right) \\
d \varpi_{2} \equiv \omega_{1} \wedge \pi_{21} \quad\left(\bmod \quad \varpi, \varpi_{1}, \varpi_{2}\right)
\end{array}\right.
$$

Thus we see, from (ii), that $R$ admitts a 1-dimensional Cauchy characteristic system in case $\mathfrak{f}^{\perp}=\left\langle\left\{e_{1} \odot e_{1}, e_{1} \odot e_{2}\right\}\right\rangle$, i.e, in case $R$ is of codimension 2 and $\mathfrak{f}$ is involutive. In fact, in [C1], E.Cartan characterized overdetermined involutive system $R$ by the condition that $R$ admitts a 1-dimensional Cauchy characteristic system. 
We will discuss the case when $R$ admitts a non-trivial Cauchy characteristic system in $\S 4.2$ in general. We will encounter the case $($ iii) in $\S 5.3$ as an example of Parabolic Geometry associated with $\mathfrak{s l}(4)$.

3.3. Involutive Symbols. In general we will consider the case when $\operatorname{dim} V=n$. We identify $S^{2}(V)$ with the dual space of $S^{2}\left(V^{*}\right)$ through the natural pairing of $S^{2}(V)$ and $S^{2}\left(V^{*}\right)$ as subspaces of $V \otimes V$ and $V^{*} \otimes V^{*}$. Then, for a basis $\left\{e_{1}, \ldots, e_{n}\right\}$ of $V$, we have a basis $\left\{e_{1}^{*} \odot e_{1}^{*}, \ldots, e_{n}^{*} \odot e_{n}^{*}, 2 e_{i}^{*} \odot e_{j}^{*}(1 \leqq i<j \leqq n)\right\}$ of $S^{2}\left(V^{*}\right)$ and its dual basis $\left\{e_{i} \odot e_{j}(1 \leqq i \leqq j \leqq n)\right\}$ of $S^{2}(V)$, where $\left\{e_{1}^{*}, \ldots, e_{n}^{*}\right\}$ is the dual basis of $V^{*}$.

Here we note the adjoint map $\sigma^{*}(v): S^{k}(V) \rightarrow S^{k+1}(V)$ of the interior multiplication $\sigma(v): S^{k+1}\left(V^{*}\right) \rightarrow S^{k}\left(V^{*}\right)$ by a vector $\left.v \in V ; \sigma(v)(f)=v\right\rfloor f$, i.e., $\sigma(v)(f)\left(v_{1}, \ldots, v_{k}\right)=$ $f\left(v, v_{1}, \ldots, v_{k}\right)$ for $f \in S^{k+1}\left(V^{*}\right)$, is given by

$$
\sigma^{*}(v)(a)=v \odot a \quad \text { for } \quad a \in S^{k}(V) .
$$

Hence, for a subspace $\mathfrak{f} \subset S^{2}\left(V^{*}\right)$ such that $\mathfrak{f}^{\perp}=\left\langle\left\{f_{1}, \ldots, f_{s}\right\}\right\rangle$, the first prolongation $\mathfrak{f}^{(1)}=\mathfrak{f} \otimes V^{*} \cap S^{3}\left(V^{*}\right)$ is given by $\left(\mathfrak{f}^{(1)}\right)^{\perp}=\left\langle\left\{e_{i} \odot f_{j} \mid 1 \leqq i \leqq n, \quad 1 \leqq j \leqq s\right\}\right\rangle$.

As in $\S 3.2$, we can classify codimension 1 subspace $\mathfrak{f} \subset S^{2}\left(V^{*}\right)$ as follows; In this case, we can classify a generator $f$ of $\mathfrak{f}^{\perp}$ as a quadratic form and obtain

$$
f=e_{1} \odot e_{1} \pm e_{2} \odot e_{2} \pm \cdots \pm e_{r} \odot e_{r}
$$

for a basis $\left\{e_{1}, \ldots, e_{n}\right\}$ of $V$, where $r$ is the rank of $f$ and we have a index when we classify over $\mathbb{R}$. In each case, $\left\{e_{n}, \ldots, e_{1}\right\}$ forms a regular basis for $\mathfrak{f}$ and the Cartan characters are given by $s_{i}=n-i+1$ for $i=1, \ldots, n-1$ and $s_{n}=0 . \mathfrak{f}$ is always involutive.

For a single equation of second order

$$
R=\left\{F\left(x_{1}, \ldots, x_{n}, z, p_{1}, \ldots, p_{n}, p_{11}, \ldots, p_{n n}\right)=0\right\} \subset L(J),
$$

we observe the following: From $\S 2.5$, we calculate

$$
\left[\left[\frac{\partial}{\partial p_{i i}}, X\right], X\right]=v_{i}^{2} \frac{\partial}{\partial z}, \quad\left[\left[\frac{\partial}{\partial p_{i j}}, X\right], X\right]=2 v_{i} \cdot v_{j} \frac{\partial}{\partial z} \quad(i \neq j),
$$

for $X=\sum_{i=1}^{n} v_{i} \frac{d}{d x_{i}}$. Thus $\frac{\partial}{\partial p_{i i}}$ is identified with $e_{i}^{*} \odot e_{i}^{*}$ in $S^{2}\left(V^{*}\right)$ and $\frac{\partial}{\partial p_{i j}}$ is identified with $2 e_{i}^{*} \odot e_{j}^{*}$ in $S^{2}\left(V^{*}\right)$, where $\left\{e_{i}=\frac{d}{d x_{i}}(i=1, \ldots, n)\right\}$ forms a basis of $V$. Then, from $\mathfrak{f}(v)=T_{v}(R) \cap \mathrm{Ch}\left(C^{2}\right)(v)$ at $v \in R$ and

$$
d F \equiv \sum_{1 \leqq i \leqq j \leqq n} \frac{\partial F}{\partial p_{i j}} d p_{i j} \quad\left(\bmod \quad \varpi, \varpi_{1}, \ldots, \varpi_{n}, d x_{1}, \ldots, d x_{n}\right),
$$

we see that $(\mathfrak{f}(v))^{\perp}$ is generated by

$$
f=\sum_{1 \leqq i \leqq j \leqq n} \frac{\partial F}{\partial p_{i j}}(v) e_{i} \odot e_{j} .
$$

Next we consider the case when $\operatorname{codim} \mathfrak{f}=2$. In this case the involutiveness becomes rather a restrictive condition and, in fact, we have (cf. [C2] and [Y2])

Proposition 3.1. Let $\mathfrak{f}$ be a subspace of $S^{2}\left(V^{*}\right)$ of codimension 2. Then $\mathfrak{f}$ is involutive if and only if there exists a basis $\left\{e_{1}, \ldots, e_{n}\right\}$ of $V$ such that the annihilator $\mathfrak{f}^{\perp}$ of $\mathfrak{f}$ in $S^{2}(V)$ is generated by $e_{1} \odot e_{2}$ and $e_{1} \odot e_{3}$ or by $e_{1} \odot e_{1}$ and $e_{1} \odot e_{2}$. 
Proof. Fist we observe that $S^{2}\left(V^{*}\right)$ is a involutive subspace of $V^{*} \otimes V^{*}$ with the Cartan characters $\sigma_{i}=n-i+1$ for $i=1, \ldots, n$. Let $\mathfrak{f}$ be a subspace of $S^{2}\left(V^{*}\right)$ of codimension 2 and let $s_{1}, \ldots s_{n}$ be the Cartan characters of $\mathfrak{f}$. Then we have $s_{i} \leqq \sigma_{i}$ for $i=1, \ldots, n$ and

$$
\operatorname{dim} \mathfrak{f}=s_{1}+\cdots+s_{n}=\sigma_{1}+\cdots+\sigma_{n}-2 .
$$

Since the Cartan characters have the property $s_{1} \geqq \cdots \geqq s_{n}$, if $s_{i_{o}}=\sigma_{i_{o}}-2$ for some $i_{o} \in\{1, \ldots, n\}$, it follows $\mathfrak{s}_{i_{o}+1}=\sigma_{i_{o}+1}=n-i_{o}>s_{i_{o}}=\sigma_{i_{o}}-2=n-i_{o}-1$, which is a contradiction. Hence there exist $j$ and $k(1 \leqq j<k \leqq n)$ such that $s_{j}=\sigma_{j}-1$ and $s_{k}=\sigma_{k}-1$.

Now assume that $\mathfrak{f}$ is involutive. Then, from $\operatorname{dim} \mathfrak{f}^{(1)}=s_{1}+2 s_{2}+\cdots+n s_{n}$, we obtain

$$
\operatorname{codim} \mathfrak{f}^{(1)}=\sum_{i=1}^{n} i\left(\sigma_{i}-s_{i}\right)=j+k \leqq(n-1)+n=2 n-1 .
$$

This implies that the generator $\left\{e_{1} \odot f, \ldots, e_{n} \odot f, e_{1} \odot g, \ldots, e_{n} \odot g\right\}$ of $\left(\mathfrak{f}^{(1)}\right)^{\perp}$ in $S^{3}(V)$ are linearly dependent, where $f$ and $g \in S^{2}(V)$ are the generator of $\mathfrak{f}^{\perp}$. Namely there exist $v_{1}$ and $v_{2} \in V$ such that

$$
v_{1} \odot f-v_{2} \odot g=0 .
$$

Here $v_{1}$ and $v_{2}$ are linearly independent since $f$ and $g$ are independent. Hence there exists $v \in V$ such that $f=v_{2} \odot v$ and $g=v_{1} \odot v$. In case $\left\{v_{1}, v_{2}, v\right\}$ are independent, there exists a basis $\left\{e_{1}, \ldots, e_{n}\right\}$ of $V$ such that $f=e_{1} \odot e_{2}$ and $g=e_{1} \odot e_{3}$. In case $\left\{v_{1}, v_{2}, v\right\}$ are dependent, there exists a basis of $V$ such that $\mathfrak{f}^{\perp}$ is generated by $e_{1} \odot e_{1}$ and $e_{1} \odot e_{2}$. Consequently, in these cases, we have $s_{i}=n-i+1$ for $1 \leqq i \leqq n-2, s_{n-1}=1$ and $s_{n}=0$.

In [C2], E.Cartan, in fact, first classified involutive subspaces $\mathfrak{f} \subset S^{2}\left(V^{*}\right)$ when $\operatorname{dim} V=$ 3 and immediately wrote the Structure Equation for each involutive system in this case.

However we cannot pursue this line in general by the following facts. By counting the dimensions, we see that the dimension of $\operatorname{Gr}\left(S^{2}\left(V^{*}\right), r\right)$ exceeds the dimension of $G L(V)$ for $n \geqq 4$ and $r, s \geqq 2$, where $r+s=\operatorname{dim} \operatorname{Gr}\left(S^{2}\left(V^{*}\right), r\right)$. Hence we will have a functional moduli if we try to classify $r$ dimensional subspaces $\mathfrak{f}$ in $S^{2}\left(V^{*}\right)$ for $n \geqq 4$ and $r, s \geqq 2$. We suspect this phenomena even if we assume the involutiveness of $\mathfrak{f}$.

Thus we need other guide lines to proceed. In this paper, after preparing the structure theory for submanifolds in $L(J)$ in $\S 4$, we will utilize Parabolic Geometries to find good classes of second order equations in $\S 5$ and $\S 6$.

3.4. Typical Symbols. We exhibit here typical examples of involutive symbols in $S^{2}\left(V^{*}\right)$, which are the only invariants of the correspondig involutive systems of second order, which were found in [Y4]. Namely we describe here the involutive subspaces $\mathfrak{f}^{1}(r), \mathfrak{f}^{2}(r)$ and $\mathfrak{f}^{3}(r)$ of $S^{2}\left(V^{*}\right)$ which have the following property: Let $R$ be an involutive systems of second order, which is regular of type $\mathfrak{f}$, i.e., $R$ satisfies the condition $(C)$ such that the symbol $\mathfrak{f}(v)$ at each point $v \in R$ is isomorphic to $\mathfrak{f} \subset S^{2}\left(V^{*}\right)$, where $\mathfrak{f}$ is one of $\mathfrak{f}^{1}(r), \mathfrak{f}^{2}(r)$ or $f^{3}(r)$. Then, as in the case of the system of first order partial differential equations of one dependent variable, $R$ can be transformed to the model linear equation by a contact transformation(cf. [Y4]).

$$
\begin{aligned}
\mathfrak{f}^{1}(r) \subset S^{2}\left(V^{*}\right) \quad(2 \leqq r \leqq n-2) \quad V=V_{r} \oplus V_{s} \\
\left(\mathfrak{f}^{1}(r)\right)^{\perp}=\left\langle\left\{e_{i} \odot e_{\alpha} \mid 1 \leqq i \leqq r, r+1 \leqq \alpha \leqq n\right\}\right\rangle=V_{r} \otimes_{S} V_{s}
\end{aligned}
$$


When $\mathfrak{f}=\mathfrak{f}^{1}(r)$ (see [Y4] $\left.\S 2\right)$, there exists a canonical coordinate system $\left(x_{i}, z, p_{i}, p_{i j}\right)$ $(1 \leqq i \leqq j \leqq n)$ of $L(J)$ such that

$$
R=\left\{\frac{\partial^{2} z}{\partial x_{i} \partial x_{\alpha}}=0 \quad(1 \leqq i \leqq r, r+1 \leqq \alpha \leqq n)\right\} .
$$

(2) $\mathfrak{f}^{2}(r) \subset S^{2}\left(V^{*}\right) \quad(r \geqq 2)$

$$
\left(\mathfrak{f}^{2}(r)\right)^{\perp}=\left\langle\left\{e_{i} \odot e_{j} \mid 1 \leqq i \leqq j \leqq r\right\}\right\rangle=S^{2}\left(V_{r}\right),
$$

When $\mathfrak{f}=\mathfrak{f}^{2}(r)$ (see [Y4] $\left.\S 3\right)$, there exists a canonical coordinate system $\left(x_{i}, z, p_{i}, p_{i j}\right)$ $(1 \leqq i \leqq j \leqq n)$ of $L(J)$ such that

$$
R=\left\{\frac{\partial^{2} z}{\partial x_{i} \partial x_{j}}=0 \quad(1 \leqq i \leqq j \leqq r)\right\} .
$$

(3) $\mathfrak{f}^{3}(r) \subset S^{2}\left(V^{*}\right) \quad(r \leqq n-2)$

$$
\left(\mathfrak{f}^{3}(r)\right)^{\perp}=\left\langle\left\{e_{i} \odot e_{a} \mid 1 \leqq i \leqq r, 1 \leqq a \leqq n\right\}\right\rangle=V_{r} \otimes_{S} V,
$$

When $\mathfrak{f}=\mathfrak{f}^{3}(r)$ (see $\left.[\mathrm{Y} 4] \S 4\right)$, there exists a canonical coordinate system $\left(x_{i}, z, p_{i}, p_{i j}\right)$ $(1 \leqq i \leqq j \leqq n)$ of $L(J)$ such that

$$
R=\left\{\frac{\partial^{2} z}{\partial x_{i} \partial x_{a}}=0 \quad(1 \leqq i \leqq r, 1 \leqq a \leqq n)\right\} .
$$

Here $\left\{e_{1}, \ldots, e_{n}\right\}$ is a basis of $V, V_{r}=\left\langle\left\{e_{1}, \ldots, e_{r}\right\}\right\rangle$ and $V_{s}=\left\langle\left\{e_{r+1}, \ldots, e_{n}\right\}\right\rangle$.

We need Reduction Theorems to explain why second order equations with these symbols have the property that their symbols are the only invariants under contact transformations. We will explain this fact for the type $\mathfrak{f}^{3}(r)$ in $\S 6.1$ by utilizing the First Reduction Theorem in $\S 4$. The other cases will be explained by utilizing the two step reduction procedure in Part II.

\section{4. $P D$ MANifolds of SECOND ORder.}

We will here formulate the submanifold theory for $(L(J), E)$ as the geometry of $P D$ manifolds of second order ([Y1]) and discuss the First Reduction Theorem.

4.1. Realization Theorem. Let $R$ be a submanifold of $L(J)$ satisfying the following condition:

$$
\text { (R.0) } \quad p: R \rightarrow J ; \text { submersion, }
$$

where $p=\left.\pi\right|_{R}$ and $\pi: L(J) \rightarrow J$ is the projection. Let $D^{1}$ and $D^{2}$ be differential systems on $R$ obtained by restricting $C^{1}=\partial E$ and $C^{2}=E$ to $R$. Moreover we denote by the same symbols those 1 -forms obtained by restricting the defining 1 -forms $\left\{\varpi, \varpi_{1}, \cdots, \varpi_{n}\right\}$ of the canonical system $E$ to $R$. Then it follows from (R.0) that these 1-forms are independent at each point on $R$ and that

$$
D^{1}=\{\varpi=0\}, \quad D^{2}=\left\{\varpi=\varpi_{1}=\cdots=\varpi_{n}=0\right\} .
$$

In fact $\left(R ; D^{1}, D^{2}\right)$ further satisfies the following conditions:

(R.1) $D^{1}$ and $D^{2}$ are differential systems of codimension 1 and $n+1$ respectively.

$(R .2) \quad \partial D^{2} \subset D^{1}$.

(R.3) Ch $\left(D^{1}\right)$ is a subbundle of $D^{2}$ of codimension $n$.

$(R .4) \quad \mathrm{Ch}\left(D^{1}\right)(v) \cap \mathrm{Ch}\left(D^{2}\right)(v)=\{0\} \quad$ at each $v \in R$. 
Here $(R .2)$ follows from $d \varpi \equiv 0\left(\bmod \varpi, \varpi_{1}, \ldots, \varpi_{n}\right)$. (R.3) follows from $\operatorname{Ch}\left(D^{1}\right)=$ $\operatorname{Ker} p_{*}=\left\{d z=d x_{1}=\cdots=d x_{n}=d p_{1}=\cdots=d p_{n}=0\right\}$. Moreover the last condition follows easily from the Realization Lemma below.

Conversely these four conditions characterize submanifolds in $L(J)$ satisfying (R.0). To see this, we first recall the following Realization Lemma, which characterize submanifolds of $(J(M, n), C)$.

Realization Lemma. Let $R$ and $M$ be manifolds. Assume that the quadruple $(R, D, p, M)$ satisfies the following conditions :

(1) $p$ is a map of $R$ into $M$ of constant rank.

(2) $D$ is a differential system on $R$ such that $F=\operatorname{Ker} p_{*}$ is a subbundle of $D$ of codimension $n$.

Then there exists a unique map $\psi$ of $R$ into $J(M, n)$ satisfying $p=\pi \cdot \psi$ and $D=\psi_{*}^{-1}(C)$, where $C$ is the canonical differential system on $J(M, n)$ and $\pi: J(M, n) \rightarrow M$ is the projection. Furthermore, let $v$ be any point of $R$. Then $\psi$ is in fact defined by

$$
\psi(v)=p_{*}(D(v)) \quad \text { as a point of } G r\left(T_{p(v)}(M)\right),
$$

and satisfies

$$
\operatorname{Ker}\left(\psi_{*}\right)_{v}=F(v) \cap C h(D)(v) .
$$

where $C h(D)$ is the Cauchy Characteristic System of D.

For the proof, see Lemma 1.5 [Y1].

In view of this Lemma, we call the triplet $\left(R ; D^{1}, D^{2}\right)$ of a manifold and two differential systems on it a $P D$ manifold of secomd order if these satisfy the above four conditions $(R .1)$ to $(R .4)$. Here we note, by $(R .2)$, subbundles $D^{2}, D^{1}$ and $T(R)$ define a filtration on $R$. Hence we can form the symbol algebra $\mathfrak{s}(v)=\mathfrak{s}_{-3}(v) \oplus \mathfrak{s}_{-2}(v) \oplus \mathfrak{s}_{-1}(v)$ of $\left(R ; D^{1}, D^{2}\right)$ at $v \in R$ as in $\S 3.1$.

We have the (local) Realization Theorem for $P D$ manifolds as follows: From conditions $(R .1)$ and (R.3), it follows that the codimension of the foliation defined by the completely integrable system $\mathrm{Ch}\left(D^{1}\right)$ is $2 n+1$. Assume that $R$ is regular with respect to $\mathrm{Ch}\left(D^{1}\right)$, i.e., the space $J=R / \mathrm{Ch}\left(D^{1}\right)$ of leaves of this foliation is a manifold of dimension $2 n+1$ such that each fibre of the projection $p: R \rightarrow J=R / \mathrm{Ch}\left(D^{1}\right)$ is connected and $p$ is a submersion. Then $D^{1}$ drops down to $J$. Namely there exists a differential system $C$ on $J$ of codimension 1 such that $D^{1}=p_{*}^{-1}(C)$. From $\mathrm{Ch}(C)=\{0\},(J, C)$ becomes a contact manifold of dimension $2 n+1$. Conditions $(R .1)$ and $(R .2)$ guarantees that the image of the following map $\iota$ is a legendrian subspace of $(J, C)$ :

$$
\iota(v)=p_{*}\left(D^{2}(v)\right) \subset C(u), \quad u=p(v) .
$$

Finally the condition (R.4) shows that $\iota: R \rightarrow L(J)$ is an immersion by Realization Lemma for $\left(R, D^{2}, p, J\right)$. Furthermore we have (Corollary $5.4[\mathrm{Y} 1]$ )

Theorem 4.1. Let $\left(R ; D^{1}, D^{2}\right)$ and $\left(\hat{R} ; \hat{D}^{1}, \hat{D}^{2}\right)$ be PD manifolds of second order. Assume that $R$ and $\hat{R}$ are regular with respect to $C h\left(D^{1}\right)$ and $C h\left(\hat{D}^{1}\right)$ respectively. Let $(J, C)$ and $(\hat{J}, \hat{C})$ be the associated contact manifolds. Then an isomorphism $\Phi:\left(R ; D^{1}, D^{2}\right) \rightarrow$ $\left(\hat{R} ; \hat{D}^{1}, \hat{D}^{2}\right)$ induces a contact diffeomorphism $\varphi:(J, C) \rightarrow(\hat{J}, \hat{C})$ such that the following commutes; 


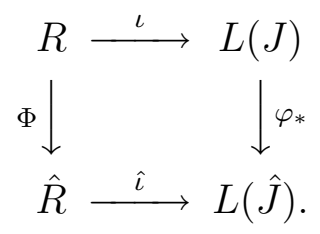

Proof. Since $\Phi$ is an isomorphism of $\left(R, D^{1}, D^{2}\right)$ onto $\left(\hat{R}, \hat{D}^{1}, \hat{D}^{2}\right)$, we have $\Phi_{*}\left(D^{1}\right)=\hat{D}^{1}$. Hence we get $\Phi_{*}\left(\operatorname{Ch}\left(D^{1}\right)\right)=\operatorname{Ch}\left(\hat{D}^{1}\right)$. Therefore, since $\operatorname{Ch}\left(D^{1}\right)=\operatorname{Ker} p_{*}$ and each fibre of $p: R \rightarrow J$ is connected, $\Phi$ is fibre-preseving and induces a unique diffeomorphism $\varphi$ of $J$ onto $\hat{J}$ such that $\hat{p} \cdot \Phi=\varphi \cdot p$. By $D^{1}=\left(p_{*}\right)^{-1}(C)$ and $\hat{D}^{1}=\left(\hat{p}_{*}\right)^{-1}(\hat{C}), \varphi$ is a contact diffeomorphism of $J$ onto $\hat{J}$, Put $\bar{\iota}=\left(\varphi_{*}\right)^{-1} \cdot \hat{\iota} \cdot \Phi$. Then it is easy to see that $\bar{\iota}$ is a map of $R$ into $L(J)$ satisfying $\pi \cdot \bar{\iota}=p$ and $D^{2}=\left(\bar{\iota}_{*}\right)^{-1}\left(C^{2}\right)$. Therefore by the uniqueness of the cannonical immersion $\iota$ of $R$ into $L(J)$, we obtain $\iota=\bar{\iota}$, i.e., $\hat{\iota} \cdot \Phi=\varphi_{*} \cdot \iota$.

By this theorem, the submanifold theory for $(L(J), E)$ is reformulated as the geometry of $P D$ manifolds of second order.

4.2. First Reduction Theorem. When $D^{1}=\partial D^{2}$ holds for a $P D$ manifold $\left(R ; D^{1}, D^{2}\right)$ of second order, the geometry of $\left(R ; D^{1}, D^{2}\right)$ reduces to that of $\left(R, D^{2}\right)$ and the Tanaka theory is directly applicable to this case. We will treat this case as Parabolic Geometries associated with $P D$ manifolds of second order in $\S 5$. Concerning about this situation, we will show the following proposition under the compatibility condition $(C)$ :

$(C) \quad p^{(1)}: R^{(1)} \rightarrow R$ is onto.

where $R^{(1)}$ is the first prolongation of $\left(R ; D^{1}, D^{2}\right)$,i.e.,

$$
R^{(1)}=\left\{n \text {-dim. integral elements of }\left(R, D^{2}\right), \text { transversal to } F=\operatorname{Ker} p_{*}\right\} \subset J(R, n),
$$

(cf. Proposition $5.11[\mathrm{Y} 1]$ ).

Proposition 4.1. Let $\left(R ; D^{1}, D^{2}\right)$ be a PD manifold of second order satisfying the condition $(C)$ above. Then the following equality holds at each point $v$ of $R$ :

$$
\operatorname{dim} D^{1}(v)-\operatorname{dim} \partial D^{2}(v)=\operatorname{dim} C h\left(D^{2}\right)(v) .
$$

In particular $D^{1}=\partial D^{2}$ holds if and only if $C h\left(D^{2}\right)=\{0\}$.

Proof. By the conditon $(C)$, there exists an n-dimensional integral element $V$ of $\left(R, D^{2}\right)$ at $v$ such that

$$
D^{2}(v)=\mathfrak{s}_{-1}(v)=V \oplus \mathfrak{f}(v) .
$$

In terms of the symbol algebra $\mathfrak{s}(v)=\mathfrak{s}_{-3}(v) \oplus \mathfrak{s}_{-2}(v) \oplus \mathfrak{s}_{-1}(v)$, we have

$$
\operatorname{Ch}\left(D^{2}\right)(v)=\left\{X \in \mathfrak{s}_{-1}(v) \mid\left[X, \mathfrak{s}_{-1}(v)\right]=0\right\}
$$

For a vector $X=w_{X}+f_{X} \in \mathrm{Ch}\left(D^{2}\right)(v)$, where $w_{X} \in V$ and $f_{X} \in \mathfrak{f}(v),[X, V]=0$ forces $f_{X}=0$, that is, $\operatorname{Ch}\left(D^{2}\right)(v) \subset V \subset \mathfrak{s}_{-1}(v)$. We put $\mathcal{C}(v)=\operatorname{Ch}\left(D^{2}\right)(v) \subset V$. Then we have

$$
\mathcal{C}(v)=\{w \in V \mid w\rfloor f=0 \text { for all } f \in \mathfrak{f}(v)\} \subset V .
$$

Thus $\mathcal{C}(v)=E$ is the largest subspace of $V$ such that $\mathfrak{f}(v) \subset S^{2}\left(E^{\perp}\right)$. On the other hand, let us consider the derived system $\partial D^{2}$ in terms of the symbol algebra $\mathfrak{s}(v)$. From $\left[\mathfrak{s}_{-1}(v), \mathfrak{s}_{-1}(v)\right]=[V, \mathfrak{f}(v)]$, putting $\mathcal{D}(v)=\pi_{2}\left(\partial D^{2}(v)\right)$, we have

$$
\mathcal{D}(v)=\{z\rfloor f \mid z \in V, f \in \mathfrak{f}(v)\} \subset V^{*},
$$

under the identification $\mathfrak{s}_{-2}(v) \cong V^{*}$, where $\pi_{2}: D^{1}(v) \rightarrow \mathfrak{s}_{-2}(v)$ is the projection. Thus $w$ belongs to the annihilator of $\mathcal{D}(v)$ iff $\langle w, z\rfloor f\rangle=0$ for all $z \in V$ and all $f \in \mathfrak{f}(v)$, hence 
iff $w\rfloor f=0$ for all $f \in \mathfrak{f}(v)$. This implies $\mathcal{D}(v)=(\mathcal{C}(v))^{\perp}$, which completes the proof of Proposition.

When a $P D$ manifold $\left(R ; D^{1}, D^{2}\right)$ admits a non-trivial Cauchy characteristics, i.e.,when rank $\mathrm{Ch}\left(D^{2}\right)>0$, the geometry of $\left(R ; D^{1}, D^{2}\right)$ is further reducible to the geometry of a single differential system. Here we will be concerned with the local equivalence of $\left(R ; D^{1}, D^{2}\right)$, hence we may assume that $R$ is regular with respect to $\mathrm{Ch}\left(D^{2}\right)$, i.e., the leaf space $X=R / \mathrm{Ch}\left(D^{2}\right)$ is a manifold such that the projection $\rho: R \rightarrow X$ is a submersion and there exists a differential system $D$ on $X$ satisfying $D^{2}=\rho_{*}^{-1}(D)$. Then the local equivalence of $\left(R ; D^{1}, D^{2}\right)$ is further reducible to that of $(X, D)$ as in the following: We assume that $\left(R ; D^{1}, D^{2}\right)$ satisfies the condition $(C)$ above and $\mathrm{Ch}\left(D^{2}\right)$ is a subbundle of rank $r(0<r<n)$. Then, by Proposition $4.1, \partial D^{2}$ is a subbundle of $D^{1}$ of codimension $r$.

By the information of the symbol algebra $\mathfrak{s}(v)$ of $\left(R ; D^{1}, D^{2}\right)$ at $v \in R$, we can find locally independent 1 -forms $\varpi, \varpi_{i}, \omega_{i}(i=1, \ldots, n)$ around $v$ such that

$$
\begin{aligned}
& D^{1}=\{\varpi=0\}, \\
& \partial D^{2}=\left\{\varpi=\varpi_{1}=\cdots=\varpi_{r}=0\right\}, \quad D^{2}=\left\{\varpi=\varpi_{1}=\cdots=\varpi_{n}=0\right\} \\
& \left\{\begin{array}{rrrl}
d \varpi & \equiv \omega_{1} \wedge \varpi_{1}+\cdots+\omega_{n} \wedge \varpi_{n}(\bmod & \varpi) \\
d \varpi_{i} \equiv 0 & \left(\bmod \quad \varpi, \varpi_{1}, \ldots, \varpi_{n}\right) & i=1, \ldots, r \\
d \varpi_{\alpha} \neq \equiv 0 & \left(\bmod \quad \varpi, \varpi_{1}, \ldots, \varpi_{n}\right) & \alpha=r+1, \ldots, n
\end{array}\right.
\end{aligned}
$$

Thus we have

$$
\left\{\begin{aligned}
d \varpi & \equiv \omega_{r+1} \wedge \varpi_{r+1}+\cdots+\omega_{n} \wedge \varpi_{n}\left(\bmod \quad \varpi, \varpi_{1}, \ldots, \varpi_{r}\right) \\
d \varpi_{i} & \equiv \pi_{i}^{r+1} \wedge \varpi_{r+1}+\cdots+\pi_{i}^{n} \wedge \varpi_{n}\left(\bmod \quad \varpi, \varpi_{1}, \ldots, \varpi_{r}\right) \quad i=1, \ldots, r
\end{aligned}\right.
$$

for some 1 -foms $\pi_{i}^{\alpha}$. This shows that the Cartan rank of $(X, \partial D)$ (see [BCG $]$ II $\S 4$ ) equals to $s=n-r$ at $x=\rho(v) \in X$, which gives us a necessary condition for a differential system $(X . D)$ to be obtained from a $P D$ manifold $\left(R ; D^{1}, D^{2}\right)$ as $X=R / \mathrm{Ch}\left(D^{2}\right)$.

From $(X, D)$, at least locally, we can reconstruct the $P D$ manifold $\left(R ; D^{1}, D^{2}\right)$ as follows. First let us consider the collection $P(X)$ of hyperplanes $v$ in each tangent space $T_{x}(X)$ at $x \in X$ which contains the fibre $\partial D(x)$ of the derived system $\partial D$ of $D$.

$$
\begin{gathered}
P(X)=\bigcup_{x \in X} P_{x} \subset J(X, m-1), \\
P_{x}=\left\{v \in \operatorname{Gr}\left(T_{x}(X), m-1\right) \mid v \supset \partial D(x)\right\} \cong \mathbb{P}\left(T_{x}(X) / \partial D(x)\right)=\mathbb{P}^{r},
\end{gathered}
$$

where $m=\operatorname{dim} X$ and $r=\operatorname{rank} \operatorname{Ch}\left(D^{2}\right)$. Moreover $D_{X}^{1}$ is the canonical system obtained by the Grassmaniann construction and $D_{X}^{2}$ is the lift of $D$. Precisely, $D_{X}^{1}$ and $D_{X}^{2}$ are given by

$$
D_{X}^{1}(v)=\nu_{*}^{-1}(v) \supset D_{X}^{2}(v)=\nu_{*}^{-1}(D(x)),
$$

for each $v \in P(X)$ and $x=\nu(v)$, where $\nu: P(X) \rightarrow X$ is the projection. Then we have a map $\kappa$ of $R$ into $P(X)$ given by

$$
\kappa(v)=\rho_{*}\left(D^{1}(v)\right) \subset T_{x}(X),
$$

for each $v \in R$ and $x=\rho(v)$. By Realization Lemma for $\left(R, D^{1}, \rho, X\right), \kappa$ is a map of constant rank such that

$$
\operatorname{Ker} \kappa_{*}=\operatorname{Ch}\left(D^{1}\right) \cap \operatorname{Ker} \rho_{*}=\operatorname{Ch}\left(D^{1}\right) \cap \operatorname{Ch}\left(D^{2}\right)=\{0\} .
$$


Thus $\kappa$ is an immersion and, by a dimension count, in fact, a local diffeomorphism of $R$ into $P(X)$ such that

$$
\kappa_{*}\left(D^{1}\right)=D_{X}^{1} \quad \text { and } \quad \kappa_{*}\left(D^{2}\right)=D_{X}^{2} .
$$

Namely $\kappa:\left(R, D^{1}, D^{2}\right) \rightarrow\left(P(X), D_{X}^{1}, D_{X}^{2}\right)$ is a local isomorphism of $P D$ manifolds of second order. Thus $\left(R ; D^{1}, D^{2}\right)$ is reconstructed from $(X, D)$, at least locally, as a part of $\left(P(X) ; D_{X}^{1}, D_{X}^{2}\right)$. (Precisely, in general, $\left(P(X), D_{X}^{1}, D_{X}^{2}\right)$ becomes a $P D$ manifold on an open subset. See Proposition 4.2.) By the construction of $\left(P(X) ; D_{X}^{1}, D_{X}^{2}\right)$, an isomorphism of $(X, D)$ naturally lifts to an isomorphism of $\left(P(X) ; D_{X}^{1}, D_{X}^{2}\right)$.

Summarizing the above consideration, we obtain the following First Reduction Theorem for $P D$ manifolds admitting non-trivial Cauchy characteristics.

Theorem 4.2. Let $\left(R, D^{1}, D^{2}\right)$ and $\left(\hat{R} ; \hat{D}^{1}, \hat{D}^{2}\right)$ be PD manifolds satisfying the condition $(C)$ such that $C h\left(D^{2}\right)$ and $C h\left(\hat{D}^{2}\right)$ are subbundles of rank $r(0<r<n)$. Assume that $R$ and $\hat{R}$ are regular with respect to $C h\left(D^{2}\right)$ and $C h\left(\hat{D}^{2}\right)$ respectively. Let $(X, D)$ and $(\hat{X}, \hat{D})$ be the leaf spaces, where $X=R / C h\left(D^{2}\right)$ and $\hat{X}=\hat{R} / C h\left(\hat{D}^{2}\right)$. Let us fix points $v_{o} \in R$ and $\hat{v}_{o} \in \hat{R}$ and put $x_{o}=\rho\left(v_{o}\right)$ and $\hat{x}_{o}=\hat{\rho}\left(\hat{v}_{o}\right)$. Then a local isomorphism $\psi:\left(R ; D^{1}, D^{2}\right) \rightarrow\left(\hat{R} ; \hat{D}^{1}, \hat{D}^{2}\right)$ such that $\psi\left(v_{o}\right)=\hat{v}_{o}$ induces a local isomorphism $\varphi:$ $(X, D) \rightarrow(\hat{X}, \hat{D})$ such that $\varphi\left(x_{o}\right)=\hat{x}_{o}$ and $\varphi_{*}\left(\kappa\left(x_{o}\right)\right)=\hat{\kappa}\left(\hat{x}_{o}\right)$, and vice versa.

4.3. Construction of $\left(R(X) ; D_{X}^{1}, D_{X}^{2}\right)$. Now we will characterize differential systems $(X, D)$, which are obtained by the First Reduction Theorem from $P D$ maifolds $\left(R ; D^{1}, D^{2}\right)$ as $X=R / \mathrm{Ch}\left(D^{2}\right)$. We already saw that the necessary condition for $(X, D)$ is that $\partial D$ is of Cartan rank $s=n-r$. We will show that this condition is also sufficient .

Let $(X, D)$ be a differential system satisfying the following conditions;

$(X .1) D$ is a differential system of codimension $n+1$ such that $C h(D)$ is trivial.

$(X .2) \partial D$ is a differential system of codimension $r+1$.

(X.3) $\partial D$ is of Cartan rank $s=n-r$.

Under the conditions $(X .1)$ and $(X .2)$, Cartan rank of $\partial D$ is less than or equal to $s$ (see the proof of Propositon 4.2 below). Thus $(X .3)$ is a nondegeneracy condition for $(X, D)$.

We form the weak symbol algebra $\mathfrak{t}(x)$ of $(X, D)$ at $x \in X$ as follows:Put $\mathfrak{t}_{-3}(x)=$ $T_{x}(X) / \partial D(x), \mathfrak{t}_{-2}(x)=\partial D(x) / D(x)$ and $\mathfrak{t}_{-1}(x)=D(x)$. The subbundles $D, \partial D$ and $T(X)$ give a filtration on $X$. Hence as in the symbol algebra of PD manifolds, we can introduce the Lie brackets in

$$
\mathfrak{t}(x)=\mathfrak{t}_{-3}(x) \oplus \mathfrak{t}_{-2}(x) \oplus \mathfrak{t}_{-1}(x),
$$

so that $\mathfrak{t}(x)$ becomes a graded Lie algebra.

Now let us consider the collection $P(X)$ of hyperplanes $v$ in each tangent space $T_{x}(X)$ at $x \in X$ which contains the fibre $\partial D(x)$ of the derived system $\partial D$ of $D$.

$$
\begin{gathered}
P(X)=\bigcup_{x \in X} P_{x} \subset J(X, m-1), \\
P_{x}=\left\{v \in \operatorname{Gr}\left(T_{x}(X), m-1\right) \mid v \supset \partial D(x)\right\} \cong \mathbb{P}\left(T_{x}(X) / \partial D(x)\right)=\mathbb{P}^{r},
\end{gathered}
$$

where $m=\operatorname{dim} X$ and $r+1=\operatorname{codim} \partial D$. Moreover $D_{X}^{1}$ is the canonical system obtained by the Grassmaniann construction and $D_{X}^{2}$ is the lift of $D$. In fact, $D_{X}^{1}$ and $D_{X}^{2}$ are given by

$$
D_{X}^{1}(v)=\nu_{*}^{-1}(v) \supset D_{X}^{2}(v)=\nu_{*}^{-1}(D(x)),
$$


for each $v \in P(X)$ and $x=\nu(v)$, where $\nu: P(X) \rightarrow X$ is the projection. For a point $v \in P(X)$, we define the symbol subspace $\hat{\mathfrak{f}}(v)$ of $D(x)$ by

$$
\hat{\mathfrak{f}}(v)=\left\{X \in \mathfrak{t}_{-1}(x) \mid\left[X, \mathfrak{t}_{-2}(x)\right] \subset \hat{v}\right\},
$$

where $x=\nu(v) \in X, \hat{v}=p_{-3}(v) \subset \mathfrak{t}_{-3}(x)$ and $p_{-3}: T_{x}(X) \rightarrow \mathfrak{t}_{-3}=T_{x}(X) / \partial D(x)$ is the projection. We put

$$
R(X)=\{v \in P(X) \mid \operatorname{codim} \hat{\mathfrak{f}}(v)=s\}
$$

$(R(X)$ is an open subset of $P(X)$ under the condition (X.3). See below). We denote the restrictions of differential systems $D_{X}^{1}$ and $D_{X}^{2}$ of $P(X)$ to $R(X)$ by the same symbols.

Then we have

Proposition 4.2. $\left(R(X), D_{X}^{1}, D_{X}^{2}\right)$ is a PD manifold of second order.

Proof. By $(X .1)$ and the construction of $P(X)$, it follows that $D_{X}^{1}$ and $D_{X}^{2}$ are differential systems on $P(X)$ of codimension 1 and $n+1$ respectively. Moreover $\partial D_{X}^{2} \subset D_{X}^{1}$ holds on $P(X)$ by construction. Since $P(X)$ is a submanifold of $J(X, m-1)$, Realization Lemma for $\left(P(X) ; D_{X}^{1}, \nu, X\right)$ implies

$$
\operatorname{Ker} \bar{\iota}_{*}=\operatorname{Ker} \nu_{*} \cap \operatorname{Ch}\left(D_{X}^{1}\right)=\operatorname{Ch}\left(D_{X}^{1}\right) \cap \operatorname{Ch}\left(D_{X}^{2}\right)=\{0\},
$$

where $\bar{\iota}: P(X) \rightarrow J(X, m-1)$ is the inclusion. Thus it remains to show that $R(X)$ is an open subset of $P(X)$ under the condition $(X .3)$ and $\mathrm{Ch}\left(D_{X}^{1}\right)$ is a subbundle of $D_{X}^{2}$ of codimension $n$ on $R(X)$.

For this purpose, let us take a point $v_{o} \in P(X)$. We can find locally independent 1 -forms $\varpi_{0}, \ldots, \varpi_{r}, \pi_{1}, \ldots, \pi_{s}$ on a neighborhood $U$ of $x_{o}=\nu\left(v_{o}\right) \in X$ such that

$$
\partial D=\left\{\varpi_{0}=\cdots=\varpi_{r}=0\right\}, \quad D=\left\{\varpi_{0}=\cdots=\varpi_{r}=\pi_{1}=\cdots=\pi_{s}=0\right\} .
$$

Here we may assume that $v_{o}=\left\{\varpi_{0}=0\right\} \subset T_{x_{o}}(X)$. Then we have

$$
d \varpi_{i} \equiv 0 \quad\left(\bmod \quad \varpi_{0}, \ldots, \varpi_{r}, \pi_{1}, \ldots, \pi_{s}\right) \quad \text { for } i=0, \ldots, r .
$$

Hence there exist 1-forms $\beta_{i}^{\alpha}$ such that

$$
d \varpi_{i} \equiv \beta_{i}^{1} \wedge \pi_{1}+\cdots+\beta_{i}^{s} \wedge \pi_{s} \quad\left(\bmod \quad \varpi_{0}, \ldots, \varpi_{r}\right) \quad \text { for } i=0, \ldots, r .
$$

Thus the Cartan rank of $\partial D$ is less than or equal to $s$.

Now let us consider

$$
\varpi=\varpi_{0}+\lambda_{1} \varpi_{1}+\cdots+\lambda_{r} \varpi_{r}
$$

on $U$. Namely we consider a point $v \in P(X)$ such that $v=\{\varpi=0\} \subset T_{x}(X)$, where $x=\nu(v) \in U$. Here $\left(\lambda_{1}, \ldots, \lambda_{r}\right)$ constitutes an inhomogeneous coordinate of the fibres of $\nu: P(X) \rightarrow X$. Denoting the pullback on $P(X)$ of 1 -forms on $X$ by the same symbol, we have

$$
D_{X}^{1}=\{\varpi=0\},
$$

and

$$
d \varpi=d \varpi_{0}+\sum_{i=1}^{r} \lambda_{i} d \varpi_{i}+\sum_{i=1}^{r} d \lambda_{i} \wedge \varpi_{i}
$$

on $\nu^{-1}(U)$. From $d \varpi_{i} \equiv \sum_{\alpha=1}^{s} \beta_{i}^{\alpha} \wedge \pi_{\alpha}+\sum_{j=1}^{r} \gamma_{i}^{j} \wedge \varpi_{j} \quad(\bmod \varpi)$ for $i=0, \ldots, r$, we calculate

$$
d \varpi \equiv \sum_{\alpha=1}^{s}\left(\beta_{0}^{\alpha}+\sum_{i=1}^{r} \lambda_{i} \beta_{i}^{\alpha}\right) \wedge \pi_{\alpha}+\sum_{i=1}^{r}\left(d \lambda_{i}+\gamma_{0}^{i}+\sum_{j=1}^{r} \lambda_{j} \gamma_{j}^{i}\right) \wedge \varpi_{i} \quad(\bmod \varpi) .
$$


For a vector $Y \in \partial D(x)$, we have

$$
Y\rfloor d \varpi \equiv-\sum_{\alpha=1}^{s} \pi_{\alpha}(Y)\left(\beta_{0}^{\alpha}+\sum_{i=1}^{r} \lambda_{i} \beta_{i}^{\alpha}\right) \quad\left(\bmod \quad \varpi_{0}, \ldots, \varpi_{r}, \pi_{1}, \ldots, \pi_{s}\right) .
$$

By the definition of brackets in the weak symbol algebra $\mathfrak{t}(x)$, it follows that

$$
\hat{\mathfrak{f}}(v)=\left\{X \in D(x) \mid\left(\beta_{0}^{\alpha}+\sum_{i=1}^{r} \lambda_{i} \beta_{i}^{\alpha}\right)(X)=0 \quad \text { for } \alpha=1, \ldots, s\right\} .
$$

Hence we see that codim $\hat{\mathfrak{f}}(v)=s$ if and only if $\left\{\beta_{0}^{\alpha}+\sum_{i=1}^{r} \lambda_{i} \beta_{i}^{\alpha}\right\}_{\alpha=1}^{s}$ are independent $\left(\bmod \varpi_{0}, \ldots, \varpi_{r}, \pi_{1}, \ldots, \pi_{s}\right)$ at $x \in X$. Thus $R(X)$ is a non-empty open subset of $P(X)$ under the condition $(X .3)$. Moreover we have, at each $v \in R(X)$,

$$
\begin{aligned}
\mathrm{Ch}\left(D_{X}^{1}\right)(v)=\left\{\varpi=\pi_{\alpha}=\beta_{0}^{\alpha}+\sum_{i=1}^{r} \lambda_{i} \beta_{i}^{\alpha}=\varpi_{i}=d \lambda_{i}+\gamma_{0}^{i}+\sum_{j=1}^{r} \lambda_{j} \gamma_{j}^{i}=0\right. \\
(i=1, \ldots, r, \quad \alpha=1, \ldots, s)\} .
\end{aligned}
$$

Therefore $\mathrm{Ch}\left(D_{X}^{1}\right)$ is a subbundle of $D_{X}^{2}$ of codimension $n$ on $R(X)$, which completes the proof of Proposition.

4.4. Symbol Subspaces. We will consider the relation between the symbol subspaces $\mathfrak{f}(v)=\operatorname{Ch}\left(D_{X}^{1}\right)(v)$ and $\hat{\mathfrak{f}}(v) \in D(x)$ for a point $v \in R(X)$ and $x=\nu(v) \in X$.

First observe that there exists an integral element $V$ of $\left(R(X), D_{X}^{2}\right)$ at $v$ such that

$$
\mathfrak{s}_{-1}(v)=V \oplus \mathfrak{f}(v)\left(=D_{X}^{2}(v)\right),
$$

if and only if there exists an integral element $W$ of $(X, D)$ at $x$ such that

$$
\mathfrak{t}_{-1}(x)=W \oplus \hat{\mathfrak{f}}(v)(=D(x)) .
$$

In fact $W=\nu_{*}(V)$ and $V=\nu_{*}^{-1}(W)$, where $\nu_{*}: D_{X}^{2}(v) \rightarrow D(x)$ is onto, $\operatorname{Ker} \nu_{*}=$ $\mathrm{Ch}\left(D_{X}^{2}\right)(v)$ and $\nu_{*}: \mathfrak{f}(v) \rightarrow \hat{\mathfrak{f}}(v)$ is a linear isomorphism.

Put $\hat{\mathfrak{t}}_{-3}(v)=\mathfrak{t}_{-3}(x) / \hat{v}$, and let us consider

$$
\hat{\mathfrak{t}}(v)=\hat{\mathfrak{t}}_{-3}(v) \oplus \mathfrak{t}_{-2}(x) \oplus \mathfrak{t}_{-1}(x) .
$$

$\hat{\mathfrak{t}}(v)$ is a quotient algebra of $\mathfrak{t}(x)$ and $\hat{\mathfrak{f}}(v)=\left\{X \in \mathfrak{t}_{-1}(x) \mid\left[X, \mathfrak{t}_{-2}(x)\right]=0\right\}$ in $\hat{\mathfrak{t}}(v)$. Let us fix a basis of $\hat{\mathfrak{t}}_{-3}(v)$. Then, as in the proof of Proposition 4.2 , the basis of $\mathfrak{s}_{-3}(v)$ is fixed. Hence we have $\mathfrak{s}_{-3}(v) \cong \mathbb{R}, \mathfrak{s}_{-2}(v) \cong V^{*}$ and $\mathfrak{f}(v) \subset S^{2}\left(V^{*}\right)$. Moreover we have $\hat{\mathfrak{t}}_{-3}(v) \cong \mathbb{R}, \mathfrak{t}_{-2}(x) \cong W^{*}$ and the map $\mu: \hat{\mathfrak{f}}(v) \rightarrow S^{2}\left(W^{*}\right)$ is defined by

$$
\mu(\tilde{f})\left(w_{1}, w_{2}\right)=\left[\left[\tilde{f}, w_{1}\right], w_{2}\right] \in \mathbb{R} \cong \hat{\mathfrak{t}}_{-3}(v) \quad \text { for } \quad \tilde{f} \in \hat{\mathfrak{f}}(v) .
$$

Here we note that there exists a unique $f \in \mathfrak{f}(v)$ such that $\tilde{f}=\nu_{*}(f)$ and we obtain, by $\mathfrak{f}(v) \subset S^{2}\left(E^{\perp}\right)$ and the definition of the brackets of $\mathfrak{s}(v)$ and $\hat{\mathfrak{t}}(v)$,

$$
\left[f, v_{1}\right]=\kappa^{*}\left(\left[\tilde{f}, w_{1}\right]\right) \subset V^{*},
$$

where $w_{1}=\kappa\left(v_{1}\right), \kappa^{*}: W^{*} \rightarrow V^{*}, \kappa=\nu_{*}: V \rightarrow W$ and Ker $\kappa=E, \kappa^{*}\left(W^{*}\right)=E^{\perp}$. Moreover we have

$$
\left[\left[f, v_{1}\right], v_{2}\right]=\left[\left[\tilde{f}, w_{1}\right], w_{2}\right] \quad \text { for } \quad w_{2}=\kappa\left(v_{2}\right) .
$$

Namely $\mu$ is injective and $\kappa_{2}^{*}(\hat{\mathfrak{f}}(v))=\mathfrak{f}(v) \subset S^{2}\left(E^{\perp}\right) \subset S^{2}\left(V^{*}\right)$ where $\kappa_{2}^{*}: S^{2}\left(W^{*}\right) \rightarrow$ $S^{2}\left(V^{*}\right)$ is induced from $\kappa^{*}: W^{*} \rightarrow V^{*}$. 
Next we consider the algebraic prolongation $\mathfrak{f}(v)^{(1)} \subset S^{3}\left(V^{*}\right)$ of $\mathfrak{f}(v) \subset S^{2}\left(V^{*}\right)$,

$$
\mathfrak{f}(v)^{(1)}=\mathfrak{f}(v) \otimes V^{*} \cap S^{3}\left(V^{*}\right) .
$$

Then, since $\mathfrak{f}(v) \subset S^{2}\left(E^{\perp}\right)$, we observe $\mathfrak{f}(v)^{(1)} \subset S^{3}\left(E^{\perp}\right)$. Therefore, for the prolongation $\hat{\mathfrak{f}}(v)^{(1)}=\hat{\mathfrak{f}}(v) \otimes W^{*} \cap S^{3}\left(W^{*}\right)$ of $\hat{\mathfrak{f}}(v) \subset S^{2}\left(W^{*}\right)$, we get

$$
\kappa_{3}^{*}\left(\hat{\mathfrak{f}}(v)^{(1)}\right)=\mathfrak{f}(v)^{(1)} \subset S^{3}\left(E^{\perp}\right) .
$$

where $\kappa_{3}^{*}: S^{3}\left(W^{*}\right) \rightarrow S^{3}\left(V^{*}\right)$ is induced from $\kappa^{*}: W^{*} \rightarrow V^{*}$. Repeatedly we obtain $\kappa_{k+2}^{*}\left(\hat{\mathfrak{f}}(v)^{(k)}\right)=\mathfrak{f}(v)^{(k)} \subset S^{k+2}\left(E^{\perp}\right)$ for the higher prolongations.

Moreover, from $\mathfrak{f}(v) \subset S^{2}\left(E^{\perp}\right)$, we also observe that, for a regular basis $\left\{w_{1}, \ldots, w_{s}\right\}$ of $W$ for $\hat{\mathfrak{f}}(v) \subset S^{2}\left(W^{*}\right)$, we obtain the regular basis $\left\{v_{1}, \ldots, v_{s}, v_{s+1}, \ldots, v_{n}\right\}$ of $V$ for $\mathfrak{f}(v) \subset S^{2}\left(V^{*}\right)$ by taking $v_{i} \in V$ such that $\kappa\left(v_{i}\right)=w_{i}(i=1, \ldots, s)$ and adding a basis $\left\{v_{s+1}, \ldots, v_{n}\right\}$ of $E=$ Ker $\kappa$. In fact, in this case, we have

$$
\kappa_{2}^{*}\left(\hat{\mathfrak{f}}_{k}\right)=\mathfrak{f}_{k} \quad \text { for } k=1, \ldots, s \text { and } \mathfrak{f}_{k}=0 \text { for } k=s, \ldots, n .
$$

where $\left.\left.\hat{\mathfrak{f}}_{k}=\left\{\tilde{f} \in \hat{\mathfrak{f}}(v) \mid w_{1}\right\rfloor \tilde{f}=\cdots=w_{k}\right\rfloor \tilde{f}=0\right\}$ and $\left.\mathfrak{f}_{k}=\left\{f \in \mathfrak{f}(v) \mid v_{1}\right\rfloor f=\cdots=v_{k}\right\rfloor f=$ $0\}$.

Summarizing the above discussion, we obtain

Proposition 4.3. Notations being as above:

(1) $\mathfrak{f}(v) \subset S^{2}\left(V^{*}\right)$ is involutive if and only if $\hat{\mathfrak{f}}(v) \subset S^{2}\left(W^{*}\right)$ is involutive.

(2) $\mathfrak{f}(v) \subset S^{2}\left(V^{*}\right)$ is of finite type if and only if $\hat{\mathfrak{f}}(v) \subset S^{2}\left(W^{*}\right)$ is of finite type.

\section{Parabolic Geometries associated with $P D$ manifolds of Second Order.}

We will here exhibit Parabolic Geometries which directly correspond to the geometry of $P D$ manifolds of second order, following [Y5] and [YY2].

\subsection{Differential Systems associated with Simple Graded Lie Algebras (Para-} bolic Geometries). We first recall basic materials for simple graded Lie algebras over $\mathbb{C}$ and state the Prolongation Theorem. We will work mainly over $\mathbb{C}$ in this section for the sake of simplicity.

Let $\mathfrak{g}$ be a finite dimensional simple Lie algebra over $\mathbb{C}$. Let us fix a Cartan subalgebra $\mathfrak{h}$ of $\mathfrak{g}$ and choose a simple root system $\Delta=\left\{\alpha_{1}, \ldots, \alpha_{\ell}\right\}$ of the root system $\Phi$ of $\mathfrak{g}$ relative to $\mathfrak{h}$. Then every $\alpha \in \Phi$ is an (all non-negative or all non-positive) integer coefficient linear combination of elements of $\Delta$ and we have the root space decomposition of $\mathfrak{g}$;

$$
\mathfrak{g}=\bigoplus_{\alpha \in \Phi^{+}} \mathfrak{g}_{\alpha} \oplus \mathfrak{h} \oplus \bigoplus_{\alpha \in \Phi^{+}} \mathfrak{g}_{-\alpha},
$$

where $\mathfrak{g}_{\alpha}=\{X \in \mathfrak{g} \mid[h, X]=\alpha(h) X \quad$ for $h \in \mathfrak{h}\}$ is (1-dimensional) root space (corresponding to $\alpha \in \Phi)$ and $\Phi^{+}$denotes the set of positive roots.

Now let us take a nonempty subset $\Delta_{1}$ of $\Delta$. Then $\Delta_{1}$ defines the partition of $\Phi^{+}$as in the following and induces the gradation of $\mathfrak{g}=\bigoplus_{p \in \mathbb{Z}} \mathfrak{g}_{p}$ as follows:

$$
\begin{gathered}
\Phi^{+}=\cup_{p \geqq 0} \Phi_{p}^{+}, \quad \Phi_{p}^{+}=\left\{\alpha=\sum_{i=1}^{\ell} n_{i} \alpha_{i} \mid \sum_{\alpha_{i} \in \Delta_{1}} n_{i}=p\right\}, \\
\mathfrak{g}_{p}=\bigoplus_{\alpha \in \Phi_{p}^{+}} \mathfrak{g}_{\alpha}, \quad \mathfrak{g}_{0}=\bigoplus_{\alpha \in \Phi_{0}^{+}} \mathfrak{g}_{\alpha} \oplus \mathfrak{h} \oplus \bigoplus_{\alpha \in \Phi_{0}^{+}} \mathfrak{g}_{-\alpha}, \quad \mathfrak{g}_{-p}=\bigoplus_{\alpha \in \Phi_{p}^{+}} \mathfrak{g}_{-\alpha},
\end{gathered}
$$




$$
\left[\mathfrak{g}_{p}, \mathfrak{g}_{q}\right] \subset \mathfrak{g}_{p+q} \quad \text { for } \quad p, q \in \mathbb{Z} .
$$

Moreover the negative part $\mathfrak{m}=\bigoplus_{p<0} \mathfrak{g}_{p}$ satisfies the following generating condition :

$$
\mathfrak{g}_{p}=\left[\mathfrak{g}_{p+1}, \mathfrak{g}_{-1}\right] \text { for } \quad p<-1
$$

We denote the simple graded Lie algebra $\mathfrak{g}=\bigoplus_{p=-\mu}^{\mu} \mathfrak{g}_{p}$ obtained from $\Delta_{1}$ in this manner by $\left(X_{\ell}, \Delta_{1}\right)$, when $\mathfrak{g}$ is a simple Lie algebra of type $X_{\ell}$. Here $X_{\ell}$ stands for the Dynkin diagram of $\mathfrak{g}$ representing $\Delta$ and $\Delta_{1}$ is a subset of vertices of $X_{\ell}$. Moreover we have

$$
\mu=\sum_{\alpha_{i} \in \Delta_{1}} n_{i}(\theta)
$$

where $\theta=\sum_{i=1}^{\ell} n_{i}(\theta) \alpha_{i}$ is the highest root of $\Phi^{+}$.

Conversely we have (Theorem 3.12 [Y5])

Theorem A. Let $\mathfrak{g}=\bigoplus_{p \in \mathbb{Z}} \mathfrak{g}_{p}$ be a simple graded Lie algebra over $\mathbb{C}$ satisfying the generating condition. Let $X_{\ell}$ be the Dynkin diagram of $\mathfrak{g}$. Then $\mathfrak{g}=\bigoplus_{p \in \mathbb{Z}} \mathfrak{g}_{p}$ is isomorphic to a graded Lie algebra $\left(X_{\ell}, \Delta_{1}\right)$ for some $\Delta_{1} \subset \Delta$. Moreover $\left(X_{\ell}, \Delta_{1}\right)$ and $\left(X_{\ell}, \Delta_{1}^{\prime}\right)$ are isomorphic if and only if there exists a diagram automorphism $\phi$ of $X_{\ell}$ such that $\phi\left(\Delta_{1}\right)=\Delta_{1}^{\prime}$.

In the real case, we can utilize the Satake diagram of $\mathfrak{g}$ to describe gradations of $\mathfrak{g}$ (Theorem $3.12[\mathrm{Y} 5]$ ).

By Theorem A, the classification of the gradation $\mathfrak{g}=\bigoplus_{p \in \mathbb{Z}} \mathfrak{g}_{p}$ of $\mathfrak{g}$ satisfying the generating condition coincides with that of parabolic subalgebras $\mathfrak{g}^{\prime}=\bigoplus_{p \geqq 0} \mathfrak{g}_{p}$ of $\mathfrak{g}$. Accordingly, to each simple graded Lie algebra $\left(X_{\ell}, \Delta_{1}\right)$, there corresponds a unique $R$ space $M_{\mathfrak{g}}=G / G^{\prime}$ (compact simply connected homogeneous complex manifold) (see [Y 5] $\S 4.1$ for detail). Furthermore, when $\mu \geqq 2$, there exists the $G$-invariant differential system $D_{\mathfrak{g}}$ on $M_{\mathfrak{g}}$, which is induced from $\mathfrak{g}_{-1}$, and the standard differential system $\left(M(\mathfrak{m}), D_{\mathfrak{m}}\right)$ of type $\mathfrak{m}$ becomes an open submanifold of $\left(M_{\mathfrak{g}}, D_{\mathfrak{g}}\right)$. For the Lie algebras of all infinitesimal automorphisms of $\left(M_{\mathfrak{g}}, D_{\mathfrak{g}}\right)$, hence of $\left(M(\mathfrak{m}), D_{\mathfrak{m}}\right)$, we have the following Prolongation Theorem (Theorem $5.2[\mathrm{Y} 5]$ ).

Prolongation Theorem. Let $\mathfrak{g}=\bigoplus_{p \in \mathbb{Z}} \mathfrak{g}_{p}$ be a simple graded Lie algebra over $\mathbb{C}$ satisfying the generating condition. Then $\mathfrak{g}=\bigoplus_{p \in \mathbb{Z}} \mathfrak{g}_{p}$ is the prolongation of $\mathfrak{m}=\bigoplus_{p<0} \mathfrak{g}_{p}$ except for the following three cases.

(1) $\mathfrak{g}=\mathfrak{g}_{-1} \oplus \mathfrak{g}_{0} \oplus \mathfrak{g}_{1}$ is of depth 1 .

(2) $\mathfrak{g}=\bigoplus_{p=-2}^{2} \mathfrak{g}_{p}$ is a contact gradation.

(3) $\mathfrak{g}=\bigoplus_{p \in \mathbb{Z}} \mathfrak{g}_{p}$ is isomorphic to $\left(A_{\ell},\left\{\alpha_{1}, \alpha_{i}\right\}\right)(1<i<\ell)$ or $\left(C_{\ell},\left\{\alpha_{1}, \alpha_{\ell}\right\}\right)$.

Furthermore $\mathfrak{g}=\bigoplus_{p \in \mathbb{Z}} \mathfrak{g}_{p}$ is the prolongation of $\left(\mathfrak{m}, \mathfrak{g}_{0}\right)$ except when $\mathfrak{g}=\bigoplus_{p \in \mathbb{Z}} \mathfrak{g}_{p}$ is isomorphic to $\left(A_{\ell},\left\{\alpha_{1}\right\}\right)$ or $\left(C_{\ell},\left\{\alpha_{1}\right\}\right)$.

Here $R$-spaces corresponding to the above exceptions (1), (2) and (3) are as follows: (1) correspond to compact irreducible hermitian symmetric spaces. (2) correspond to contact manifolds of Boothby type (Standard contact manifolds), which exist uniquely for each simple Lie algebra other than $\mathfrak{s l}(2, \mathbb{C})$ (see $\S 5.2$ below). In case of $(3),\left(J\left(\mathbb{P}^{\ell}, i\right), C\right)$ corresponds to $\left(A_{\ell},\left\{\alpha_{1}, \alpha_{i}\right\}\right)$ and $\left(L\left(\mathbb{P}^{2 \ell-1}\right), E\right)$ corresponds to $\left(C_{\ell},\left\{\alpha_{1}, \alpha_{\ell}\right\}\right)(1<i<\ell)$, where $\mathbb{P}^{\ell}$ denotes the $\ell$-dimensional complex projective space and $\mathbb{P}^{2 \ell-1}$ is the Standard contact manifold of type $C_{\ell}$ corresponding to $\left(C_{\ell},\left\{\alpha_{1}\right\}\right)$. Here we note that $R$-spaces corresponding to (2) and (3) are all Jet spaces of the first or second order. 
For the real version of this theorem, we refer the reader to Theorem 5.3 [Y5].

Now the Parabolic Geometry is a geometry modeled after the homogeneous space $G / G^{\prime}$, where $G$ is a (semi-)simple Lie group and $G^{\prime}$ is a parabolic subgroup of $G$ (cf. [Bai]). Precisely, in this paper, we mean, by a Parabolic Geometry, the Geometry associated with the Simple Graded Lie Algebra in the sense of N.Tanaka ([T4]).

In fact, let $\mathfrak{g}=\bigoplus_{p \in \mathbb{Z}} \mathfrak{g}_{p}$ be a simple graded Lie algebra over $\mathbb{R}$ satisfying the generating condition. Let $M$ be a manifold with a $G_{0}^{\sharp}$-structure of type $\mathfrak{m}$ in the sense of [T4] (for the precise definition, see $\S 2$ of [T4]). In [T4], under the assumption that $\mathfrak{g}$ is the prolongation of $\left(\mathfrak{m}, \mathfrak{g}_{0}\right), \mathbf{N}$. Tanaka constructed the Normal Cartan Connection $(P, \omega)$ of Type $\mathfrak{g}$ over $M$, which settles the equivalence problem for the $G_{0}^{\sharp}$-structure of type $\mathfrak{m}$ in the following sense: Let $M$ and $\hat{M}$ be two manifolds with $G_{0}^{\sharp}$-structures of type $\mathfrak{m}$. Let $(P, \omega)$ and $(\hat{P}, \hat{\omega})$ be the normal connections of type $\mathfrak{g}$ over $M$ and $\hat{M}$ respectively. Then a diffeomorphism $\varphi$ of $M$ onto $\hat{M}$ preserving the $G_{0}^{\sharp}$-structures lifts uniquely to an isomorphism $\varphi^{\sharp}$ of $(P, \omega)$ onto $(\hat{P}, \hat{\omega})$ and vice versa ([T4], Theorem 2.7).

Here we note that, if $\mathfrak{g}$ is the prolongation of $\mathfrak{m}$, a $G_{0}^{\sharp}$-structure on $M$ is nothing but a regular differential system of type $\mathfrak{m}$ (see [T4, §2.2]). Thus a Parabolic Geometry modeled after $G / G^{\prime}$ is the geometry of $P D$ manifold of second order with the symbol algebra $\mathfrak{s}=\mathfrak{s}_{-3} \oplus \mathfrak{s}_{-2} \oplus \mathfrak{s}_{-1}$, if $\mathfrak{g}$ is the prolongation of $\mathfrak{m}$ and $\mathfrak{m}$ is isomorphic to $\mathfrak{s}$.

Hence, among simple graded Lie algebras $\mathfrak{g}=\bigoplus_{p=-\mu}^{\mu} \mathfrak{g}_{p} \cong\left(X_{\ell}, \Delta_{1}\right)$, we will seek those algebras such that $\mathfrak{m}=\bigoplus_{p<0} \mathfrak{g}_{p}$ is isomorphic to the symbol algebra of $P D$ manifolds of second order. Thus a necessary condition for this is $\mu=3$ and $\operatorname{dim} \mathfrak{g}_{-3}=1$. Then, by the above construction of $\left(X_{\ell}, \Delta_{1}\right), \mathfrak{g}_{3}$ should be the highest root space. This forces $\Delta_{\theta} \subset \Delta_{1}$ where $\left(X_{\ell}, \Delta_{\theta}\right)$ is the (standard) contact gradation of $\mathfrak{g}$ (see $§ 5.2$ below). These two conditions confine the possibility of $\left(X_{\ell}, \Delta_{1}\right)$. In fact, a simple graded Lie algebra ( $\left.X_{\ell}, \Delta_{1}\right)$, which satisfies both $\mu=3$ and $\Delta_{\theta} \subset \Delta_{1}$ is one of the following: $\left(A_{\ell},\left\{\alpha_{1}, \alpha_{i}, \alpha_{\ell}\right\}\right)$ $\left(1<i \leqq\left[\frac{\ell+1}{2}\right]\right),\left(B_{\ell},\left\{\alpha_{1}, \alpha_{2}\right\}\right),\left(C_{\ell},\left\{\alpha_{1}, \alpha_{\ell}\right\}\right),\left(D_{\ell},\left\{\alpha_{1}, \alpha_{2}\right\}\right),\left(D_{\ell},\left\{\alpha_{2}, \alpha_{\ell}\right\}\right),\left(E_{6},\left\{\alpha_{1}, \alpha_{2}\right\}\right)$ and $\left(E_{7},\left\{\alpha_{1}, \alpha_{7}\right\}\right)$ up to conjugacy.

In fact, as we will see in $\S 5.3$ and $\S 5.4$, these simple graded Lie algebras $\left(X_{\ell}, \Delta_{1}\right)$ represent the Parabolic Geometries of $P D$ manifolds of second order (of finite type), except for $\left(C_{\ell},\left\{\alpha_{1}, \alpha_{\ell}\right\}\right)$, which is one of the exception in Prolongation Theorem and represents the Parabolic Geometry of third order equations of finite type (cf. [YY2] §3 Case (4)).

Furthermore, in $\S 6.2$ and $\S 6.3$, by utilizing the First Reduction Theorem, we will see more examples of Parabolic Geometry, which is associated with the geometry of $(X, D)$ in $\S 4.3$.

5.2. Standard Contact Manifolds. Each simple Lie algebra $\mathfrak{g}$ over $\mathbb{C}$ has the highest root $\theta$. Let $\Delta_{\theta}$ denote the subset of $\Delta$ consisting of all vertices which are connected to $-\theta$ in the Extended Dynkin diagram of $X_{\ell}(\ell \geqq 2)$. This subset $\Delta_{\theta}$ of $\Delta$, by the construction in $§ 5.1$, defines a gradation (or a partition of $\Phi^{+}$), which distinguishes the highest root $\theta$. Then, this gradation $\left(X_{\ell}, \Delta_{\theta}\right)$ turns out to be a contact gradation, which is unique up to conjugacy (Theorem 4.1 [Y5]). Explicitly we have $\Delta_{\theta}=\left\{\alpha_{1}, \alpha_{\ell}\right\}$ for $A_{\ell}$ type and $\Delta_{\theta}=\left\{\alpha_{\theta}\right\}$ for other types. Here $\alpha_{\theta}=\alpha_{2}, \alpha_{1}, \alpha_{2}$ for $B_{\ell}, C_{\ell}, D_{\ell}$ types respectively and $\alpha_{\theta}=\alpha_{2}, \alpha_{1}, \alpha_{8}, \alpha_{1}, \alpha_{2}$ for $E_{6}, E_{7}, E_{8}, F_{4}, G_{2}$ types respectively.

Moreover we have the adjoint (or equivalently coadjoint) representation, which has $\theta$ as the highest weight. The $R$-space $J_{\mathfrak{g}}$ corresponding to $\left(X_{\ell}, \Delta_{\theta}\right)$ can be obtained as the projectiviation of the (co-)adjoint orbit of $G$ passing through the root vector of $\theta$. By this construction, $J_{\mathfrak{g}}$ has the natural contact structure $C_{\mathfrak{g}}$ induced from the symplectic 
structure as the coadjoint orbit, which corresponds to the contact gradation $\left(X_{\ell}, \Delta_{\theta}\right)$ (cf. [Y5], §4). Standard contact manifolds $\left(J_{\mathfrak{g}}, C_{\mathfrak{g}}\right)$ were first found by Boothby ([Bo]) as compact simply connected homogeneous complex contact manifolds.

For the explicit description of the standard contact manifolds of the classical type, we refer the reader to $\S 4.3$ [Y5].

In $\S 5.3$ and $\S 5.4$, the model equation $\left(R_{\mathfrak{g}}, D_{\mathfrak{g}}^{2}\right)$ can be realized as a $R$-space orbit in $L\left(J_{\mathfrak{g}}\right)$.

Extended Dynkin Diagrams with the coefficient of Highest Root (cf. [Bu])

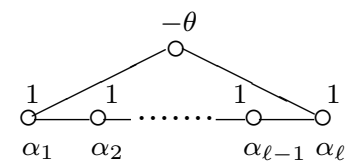

$A_{\ell}(\ell>1)$

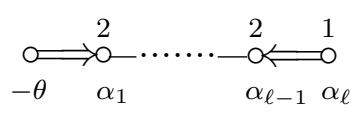

$C_{\ell}(\ell>1)$
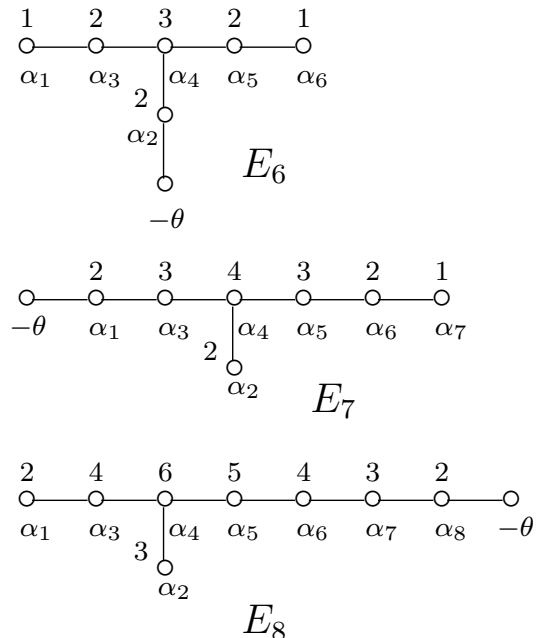

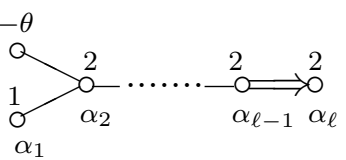

$B_{\ell}(\ell>2)$
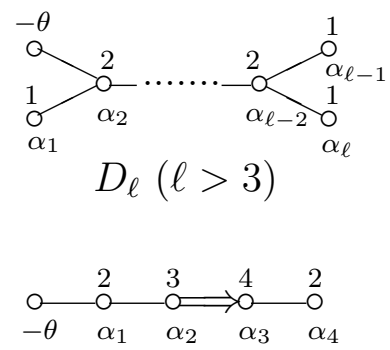

$F_{4}$

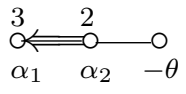

$G_{2}$

5.3. Classical Type Examples. We will describe here the symbol algebra $\mathfrak{m}=\bigoplus_{p<0} \mathfrak{g}_{p}$ of each $\left(X_{\ell}, \Delta_{1}\right)$ of the classical type and give the model equations of second order. We refer the reader to $\S 3$ of [YY2] for the detailed description of the symbol algebras in matrices form. In this and next subsections, we will discuss in the complex analytic or the real $C^{\infty}$ category depending on whether $\mathbb{K}=\mathbb{C}$ or $\mathbb{R}$.

(1) Case of $\left(A_{\ell},\left\{\alpha_{1}, \alpha_{i+1}, \alpha_{\ell}\right\}\right)\left(1<i+1 \leqq\left[\frac{\ell+1}{2}\right]\right)$.

We have the following matrix representation of $\left(A_{\ell},\left\{\alpha_{1}, \alpha_{i+1}, \alpha_{\ell}\right\}\right)$ :

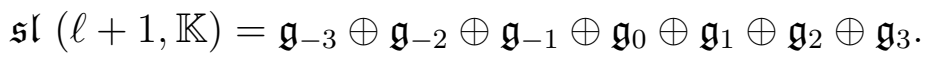


where the gradation is given by subdividing matrices as follows;

$$
\begin{aligned}
& \mathfrak{g}_{-3}=\left\{\left(\begin{array}{cccc}
0 & 0 & 0 & 0 \\
0 & 0 & 0 & 0 \\
0 & 0 & 0 & 0 \\
a & 0 & 0 & 0
\end{array}\right) \mid \quad a \in \mathbb{K}\right\} \cong \mathbb{K} \\
& \mathfrak{g}_{-2}=\left\{\left(\begin{array}{cccc}
0 & 0 & 0 & 0 \\
0 & 0 & 0 & 0 \\
-\xi_{2} & 0 & 0 & 0 \\
0 & { }^{t} \xi_{1} & 0 & 0
\end{array}\right) \mid \quad \xi_{1} \in \mathbb{K}^{i} \quad \xi_{2} \in \mathbb{K}^{j}\right\} \\
& \mathfrak{g}_{-1}=V \oplus \mathfrak{f}, \\
& \mathfrak{f}=\left\{\left(\begin{array}{cccc}
0 & 0 & 0 & 0 \\
0 & 0 & 0 & 0 \\
0 & A & 0 & 0 \\
0 & 0 & 0 & 0
\end{array}\right) \mid A \in M(j, i)\right\} \\
& V=\left\{\left(\begin{array}{cccc}
0 & 0 & 0 & 0 \\
x_{1} & 0 & 0 & 0 \\
0 & 0 & 0 & 0 \\
0 & 0 & { }^{t} x_{2} & 0
\end{array}\right) \mid \quad x_{1} \in \mathbb{K}^{i}, \quad x_{2} \in \mathbb{K}^{j}\right\} \cong \mathbb{K}^{\ell-1}, \\
& \mathfrak{g}_{0}=\left\{\left(\begin{array}{cccc}
b & 0 & 0 & 0 \\
0 & B & 0 & 0 \\
0 & 0 & C & 0 \\
0 & 0 & 0 & c
\end{array}\right) \mid \begin{array}{c}
b, c \in \mathbb{K}, B \in \mathfrak{g l}(i, \mathbb{K}), C \in \mathfrak{g l}(j, \mathbb{K}), \\
b+c+\operatorname{tr} B+\operatorname{tr} C=0
\end{array}\right\} \\
& \mathfrak{g}_{k}=\left\{{ }^{t} X \mid X \in \mathfrak{g}_{-k}\right\},(k=1,2,3),
\end{aligned}
$$

where $i+j=\ell-1$. Then we have

$$
\begin{aligned}
& \mathfrak{m}=\mathfrak{g}_{-3} \oplus \mathfrak{g}_{-2} \oplus(V \oplus \mathfrak{f}) \\
& =\left\{\left(\begin{array}{cccc}
0 & 0 & 0 & 0 \\
x_{1} & 0 & 0 & 0 \\
-\xi_{2} & A & 0 & 0 \\
a & { }^{t} \xi_{1} & { }^{t} x_{2} & 0
\end{array}\right)=\hat{a}+\check{\xi}+\hat{x}+\hat{A} \mid \begin{array}{c}
\xi=\left(\begin{array}{l}
\xi_{1} \\
\xi_{2}
\end{array}\right), x=\left(\begin{array}{l}
x_{1} \\
x_{2}
\end{array}\right) \in \mathbb{K}^{\ell-1}, \\
a \in \mathbb{K}, A \in M(j, i)
\end{array}\right\} .
\end{aligned}
$$

By calculating $[\check{\xi}, \hat{x}]$ and $[[\hat{A}, \hat{x}], \hat{x}]$, we have

$$
[\check{\xi}, \hat{x}]=\widehat{\left({ }^{t} \xi x\right)},[[\hat{A}, \hat{x}], \hat{x}]=\left(-\widehat{2^{t} x_{2} A} x_{1}\right), \quad 2^{t} x_{2} A x_{1}=\left({ }^{t} x_{1},{ }^{t} x_{2}\right)\left(\begin{array}{cc}
0 & { }^{t} A \\
A & 0
\end{array}\right)\left(\begin{array}{l}
x_{1} \\
x_{2}
\end{array}\right)
$$

Thus we have $\mathfrak{g}_{-2} \cong V^{*}$ and $\mathfrak{f}=\left\langle\left\{e_{k}^{*} \odot e_{\alpha}^{*}(1 \leqq k \leqq i, i+1 \leqq \alpha \leqq \ell-1)\right\}\right\rangle \subset S^{2}\left(V^{*}\right)$ for a basis $\left\{e_{1}, \ldots, e_{\ell-1}\right\}$ of $V$. This implies that the model equation of the second order is given by

$$
\frac{\partial^{2} z}{\partial x_{k} \partial x_{l}}=\frac{\partial^{2} z}{\partial x_{\alpha} \partial x_{\beta}}=0 \quad \text { for } 1 \leqq k, l \leqq i \quad \text { and } \quad i+1 \leqq \alpha, \beta \leqq \ell-1,
$$

where $z$ is dependent variable and $x_{1}, \ldots, x_{\ell-1}$ are independent variables.

(2) Case of $\left(B_{\ell},\left\{\alpha_{1}, \alpha_{2}\right\}\right)(\ell \geqq 3),\left(D_{\ell},\left\{\alpha_{1}, \alpha_{2}\right\}\right)(\ell \geqq 4)$. 
Similarly in this case, we have $\operatorname{dim} \mathfrak{f}=1$ and $\mathfrak{f}=\left\langle\left\{e_{1}^{*} \odot e_{1}^{*}+\cdots+e_{n}^{*} \odot e_{n}^{*}\right\}\right\rangle$ for a basis $\left\{e_{1}, \ldots, e_{n}\right\}$ of $V$. Thus we have the following model equation of the second order:

$$
\frac{\partial^{2} z}{\partial x_{p} \partial x_{q}}=\delta_{p q} \frac{\partial^{2} z}{\partial^{2} x_{1}} \quad \text { for } \quad 1 \leqq p, q \leqq n,
$$

where $z$ is dependent variable and $x_{1}, \ldots, x_{n}$ are independent variables. This equation is the embedding equation as a hypersurface for the quadric $Q^{n}$. For the explicit matrix description of the gradation, we refer the reader to Case (5) in $\S 3$ of [YY2].

(3) Case of $\left(D_{\ell},\left\{\alpha_{2}, \alpha_{\ell}\right\}\right)(\ell \geqq 4)$.

Similarly in this case, we have $\operatorname{dim} \mathfrak{f}=\frac{1}{2}(\ell-1)(\ell-2)$ and

$$
\mathfrak{f}=\left\langle\left\{\sum_{p, q=1}^{\ell-1} a_{p q}\left(e_{p}^{1}\right)^{*} \odot\left(e_{q}^{2}\right)^{*} \mid A=\left(a_{p q}\right) \in \mathfrak{o}(\ell-1)\right\}\right\rangle
$$

for a basis $\left\{e_{1}^{1}, \ldots, e_{\ell-1}^{1}, e_{1}^{2}, \ldots, e_{\ell-1}^{2}\right\}$ of $V$. Thus we have the following model equation of the second order:

$$
\frac{\partial^{2} z}{\partial x_{p}^{i} \partial x_{q}^{j}}+\frac{\partial^{2} z}{\partial x_{q}^{i} \partial x_{p}^{j}}=0 \quad \text { for } \quad 1 \leqq i, j \leqq 2, \quad 1 \leqq p<q \leqq \ell-1,
$$

where $z$ is dependent variable and $x_{1}^{1}, \ldots, x_{\ell-1}^{1}, x_{1}^{2}, \ldots, x_{\ell-1}^{2}$ are independent variables. This equation is the Plücker embedding equation for the Grassman manifold $\operatorname{Gr}(\ell+1,2)$ (see [SYY] §3). For the explicit matrix description of the gradation, we refer the reader to Case (10) in $\S 3$ of [YY2].

5.4. Exceptional Type Examples. We here only describe the model equation $\left(R_{\mathfrak{g}}, D_{\mathfrak{g}}^{2}\right)$ of second order in the form of the standard differential system of type $\mathfrak{m}$. We refer the reader to $\S 4$ of [YY2] for the detailed description of the symbol algebras by the use of the Chevalley basis of $\mathfrak{g}$.

(1) Case of $\left(E_{6},\left\{\alpha_{1}, \alpha_{2}\right\}\right)$.

The symbol algebra $\mathfrak{m}=\mathfrak{g}_{-3} \oplus \mathfrak{g}_{-2} \oplus \mathfrak{g}_{-1}$ is described as follows:

$$
\mathfrak{g}_{-3} \cong \mathbb{K}, \quad \mathfrak{g}_{-2} \cong V^{*}, \quad \mathfrak{g}_{-1}=V \oplus \mathfrak{f}, \quad \mathfrak{f} \subset S^{2}\left(V^{*}\right) \quad \text { and } \quad \operatorname{dim} V=10, \quad \operatorname{dim} \mathfrak{f}=5 .
$$

Here the standard differential system $\left(M(\mathfrak{m}), D_{\mathfrak{m}}\right)$ of type $\mathfrak{m}$ in this case is given by

$$
D_{\mathfrak{m}}=\left\{\varpi=\varpi_{1}=\varpi_{2}=\cdots=\varpi_{10}=0\right\},
$$

where

$$
\begin{array}{rlrl}
\varpi & =d z-p_{1} d x_{1}-\cdots-p_{10} d x_{10}, & & \\
\varpi_{1}=d p_{1}+q_{5} d x_{8}+q_{4} d x_{9}+q_{3} d x_{10}, & & \varpi_{2}=d p_{2}-q_{5} d x_{5}-q_{4} d x_{7}+q_{2} d x_{10}, \\
\varpi_{3}=d p_{3}+q_{5} d x_{4}+q_{4} d x_{6}+q_{1} d x_{10}, & \varpi_{4}=d p_{4}+q_{5} d x_{3}-q_{3} d x_{7}-q_{2} d x_{9}, \\
\varpi_{5}=d p_{5}-q_{5} d x_{2}+q_{3} d x_{6}-q_{1} d x_{9}, & \varpi_{6}=d p_{6}+q_{4} d x_{3}+q_{3} d x_{5}+q_{2} d x_{8}, \\
\varpi_{7}=d p_{7}-q_{4} d x_{2}-q_{3} d x_{4}+q_{1} d x_{8}, & \varpi_{8}=d p_{8}+q_{5} d x_{1}+q_{2} d x_{6}+q_{1} d x_{7}, \\
\varpi_{9}=d p_{9}+q_{4} d x_{1}-q_{2} d x_{4}-q_{1} d x_{5} & \varpi_{10}=d p_{10}+q_{3} d x_{1}+q_{2} d x_{2}+q_{1} d x_{3} .
\end{array}
$$

Here $\left(x_{1}, \ldots, x_{10}, z, p_{1}, \ldots, p_{10}, q_{1}, \ldots, q_{5}\right)$ is a coordinate system of $M(\mathfrak{m}) \cong \mathbb{K}^{26}$.

(2) Case of $\left(E_{7},\left\{\alpha_{1}, \alpha_{7}\right\}\right)$.

The symbol algebra $\mathfrak{m}=\mathfrak{g}_{-3} \oplus \mathfrak{g}_{-2} \oplus \mathfrak{g}_{-1}$ is described as follows:

$$
\mathfrak{g}_{-3} \cong \mathbb{K}, \quad \mathfrak{g}_{-2} \cong V^{*}, \quad \mathfrak{g}_{-1}=V \oplus \mathfrak{f}, \quad \mathfrak{f} \subset S^{2}\left(V^{*}\right) \quad \text { and } \quad \operatorname{dim} V=16, \operatorname{dim} \mathfrak{f}=10 .
$$


Here the standard differential system $\left(M(\mathfrak{m}), D_{\mathfrak{m}}\right)$ of type $\mathfrak{m}$ in this case is given by

$$
D_{\mathfrak{m}}=\left\{\varpi=\varpi_{1}=\varpi_{2}=\cdots=\varpi_{16}=0\right\},
$$

where

$$
\begin{aligned}
\varpi & =d z-p_{1} d x_{1}-\cdots-p_{16} d x_{16}, \\
\varpi_{1} & =d p_{1}+q_{10} d x_{11}+q_{9} d x_{12}+q_{8} d x_{14}+q_{7} d x_{15}+q_{5} d x_{16}, \\
\varpi_{2} & =d p_{2}-q_{10} d x_{9}-q_{9} d x_{10}-q_{8} d x_{13}+q_{6} d x_{15}+q_{4} d x_{16}, \\
\varpi_{3} & =d p_{3}+q_{10} d x_{6}+q_{9} d x_{8}-q_{7} d x_{13}-q_{6} d x_{14}+q_{3} d x_{16}, \\
\varpi_{4} & =d p_{4}-q_{10} d x_{5}-q_{9} d x_{7}-q_{5} d x_{13}-q_{4} d x_{14}-q_{3} d x_{15}, \\
\varpi_{5} & =d p_{5}-q_{10} d x_{4}+q_{8} d x_{8}+q_{7} d x_{10}+q_{6} d x_{12}+q_{2} d x_{16}, \\
\varpi_{6} & =d p_{6}+q_{10} d x_{3}-q_{8} d x_{7}+q_{5} d x_{10}+q_{4} d x_{12}-q_{2} d x_{15}, \\
\varpi_{7} & =d p_{7}-q_{9} d x_{4}-q_{8} d x_{6}-q_{7} d x_{9}-q_{6} d x_{11}+q_{1} d x_{16}, \\
\varpi_{8} & =d p_{8}+q_{9} d x_{3}+q_{8} d x_{5}-q_{5} d x_{9}-q_{4} d x_{11}-q_{1} d x_{15}, \\
\varpi_{9} & =d p_{9}-q_{10} d x_{2}-q_{7} d x_{7}-q_{5} d x_{8}+q_{3} d x_{12}+q_{2} d x_{14}, \\
\varpi_{10} & =d p_{10}-q_{9} d x_{2}+q_{7} d x_{5}+q_{5} d x_{6}-q_{3} d x_{11}+q_{1} d x_{14}, \\
\varpi_{11} & =d p_{11}+q_{10} d x_{1}-q_{6} d x_{7}-q_{4} d x_{8}-q_{3} d x_{10}-q_{2} d x_{13}, \\
\varpi_{12} & =d p_{12}+q_{9} d x_{1}+q_{6} d x_{5}+q_{4} d x_{6}+q_{3} d x_{9}-q_{1} d x_{13}, \\
\varpi_{13} & =d p_{13}-q_{8} d x_{2}-q_{7} d x_{3}-q_{5} d x_{4}-q_{2} d x_{11}-q_{1} d x_{12}, \\
\varpi_{14} & =d p_{14}+q_{8} d x_{1}-q_{6} d x_{3}-q_{4} d x_{4}+q_{2} d x_{9}+q_{1} d x_{10}, \\
\varpi_{15} & =d p_{15}+q_{7} d x_{1}+q_{6} d x_{2}-q_{3} d x_{4}-q_{2} d x_{6}-q_{1} d x_{8}, \\
\varpi_{16} & =d p_{16}+q_{5} d x_{1}+q_{4} d x_{2}+q_{3} d x_{3}+q_{2} d x_{5}+q_{1} d x_{7} .
\end{aligned}
$$

Here $\left(x_{1}, \ldots, x_{16}, z, p_{1}, \ldots, p_{16}, q_{1}, \ldots, q_{10}\right)$ is a coordinate system of $M(\mathfrak{m}) \cong \mathbb{K}^{43}$.

The model linear equations in $\S 5.3(2),(3)$ and $\S 5.4(1)$ and (2) appeared as the embedding equations of the corresponding symmetric spaces into the projective spaces in [SYY] and $[\mathrm{HY}]$ (see $[\mathrm{SYY}] \S 1$ ). Except for $\S 5.3(2)$, these equations have rigidity properties. As is pointed out in $\S 5$ of [YY2], by the vanishing of the second cohomology (cf. Theorem 2.7 and 2.9 [T4], Proposition 5.5 [Y5]), we observe that Parabolic Geometries associated with $\left(D_{\ell},\left\{\alpha_{2}, \alpha_{\ell}\right\}\right),\left(E_{6},\left\{\alpha_{1}, \alpha_{2}\right\}\right)$ and $\left(E_{7},\left\{\alpha_{1}, \alpha_{7}\right\}\right)$ have no local invariant. Thus the model second order equations of these cases is solely characterized by their symbols $\mathfrak{f} \subset S^{2}\left(V^{*}\right)$ under the condition $(C)$, as in the case of Typical involutive symbols in $§ 2.4$.

\section{Examples of First Reduction Theorem.}

Utilizing the First Reduction Theorem, we will discuss the Typical class of type $\mathfrak{f}^{3}(r)$ and exhibit several examples of $P D$ manifolds of second order given through Parabolic Geometries on (X.D).

6.1. Typical Class of Type $\mathfrak{f}^{3}(r)$. Let $\left(R ; D^{1}, D^{2}\right)$ be a $P D$ manifold of second order satisfying the condition $(C)$, which is regular of type $\mathfrak{f}^{3}(r)(r \leqq n-2)$. Namely $\left(R ; D^{1}, D^{2}\right)$ is a $P D$ manifold of second order such that symbol algebra $\mathfrak{s}(v)$ at each point $v \in R$ is isomorphic to $\mathfrak{s}=\mathfrak{s}_{-3} \oplus \mathfrak{s}_{-2} \oplus \mathfrak{s}_{-1}$ where

$$
\mathfrak{s}_{-3}=\mathbb{R}, \quad \mathfrak{s}_{-2}=V^{*} \quad \text { and } \quad \mathfrak{s}_{-1}=V \oplus \mathfrak{f}^{3}(r) .
$$


Then, by Lemma 1.2 [Y4], there exists a coframe $\left\{\varpi, \varpi_{a}, \omega_{a}, \pi_{\alpha \beta}(1 \leqq a \leqq n, r+1 \leqq\right.$ $\alpha, \beta \leqq n)\}$ on a neighborhood $U$ of each point $v \in R$ such that $D^{1}=\{\varpi=0\}, D^{2}=$ $\left\{\varpi=\varpi_{1}=\cdots=\varpi_{n}=0\right\}$ and that the following equalities hold:

$$
\begin{aligned}
& d \varpi \equiv \omega_{1} \wedge \varpi_{1}+\cdots+\omega_{n} \wedge \varpi_{n} \quad(\bmod \varpi), \\
& \left\{\begin{aligned}
d \varpi_{i} & \equiv 0 \\
d \varpi_{\alpha} & \equiv \omega_{r+1} \wedge \pi_{\alpha r+1}+\cdots+\omega_{n} \wedge \pi_{\alpha n} \quad\left(\bmod \varpi, \varpi_{1}, \ldots, \varpi_{n}\right),
\end{aligned}\right.
\end{aligned}
$$

for $1 \leqq i \leqq r$ and $r+1 \leqq \alpha \leqq n$ and $\pi_{\alpha \beta}=\pi_{\beta \alpha}$. Thus we see that Ch $\left(D^{2}\right)$ is a subbundle of $D^{2}$ of rank $r$. A coframe $\left\{\varpi, \varpi_{a}, \omega_{a}, \pi_{\alpha \beta}(1 \leqq a \leqq n, r+1 \leqq \alpha, \beta \leqq n)\right\}$ on $U$ satisfying $D^{1}=\{\varpi=0\}$ and $D^{2}=\left\{\varpi=\varpi_{1}=\cdots=\varpi_{n}=0\right\}$ is called adapted if it satisfies the above structure equations. Then we have (Lemma 4.1 [Y4])

Lemma A. Let $\left(R ; D^{1}, D^{2}\right)$ be as above and $r \leqq n-2$. Then there exists an adapted coframe on $U$ such that the following equalities hold:

$$
d \varpi_{i} \equiv 0 \quad\left(\bmod \quad \varpi_{1}, \ldots, \varpi_{r}\right) \quad \text { for } i=1, \ldots, r .
$$

By this lemma, for $\partial D^{2}=\left\{\varpi=\varpi_{1}=\cdots=\varpi_{r}=0\right\}$, we have

$$
\left\{\begin{array}{l}
d \varpi \equiv \omega_{r+1} \wedge \varpi_{r+1}+\cdots+\omega_{n} \wedge \varpi_{n} \quad\left(\bmod \varpi, \varpi_{1}, \ldots, \varpi_{r}\right), \\
d \varpi_{i} \equiv 0 \quad\left(\bmod \varpi, \varpi_{1}, \ldots, \varpi_{r}\right) .
\end{array}\right.
$$

Hence $\partial^{2} D^{2}=\partial^{(2)} D^{2}=\left\{\varpi_{1}=\cdots=\varpi_{r}=0\right\}$ and $B=\partial^{2} D^{2}$ is completely integrable.

Now we assume that $R$ is regular with respect to $\mathrm{Ch}\left(D^{2}\right)$, i.e., the leaf space $X=$ $R / \mathrm{Ch}\left(D^{2}\right)$ is a manifold such that the projection $\rho: R \rightarrow X$ is a submersion and there exists differential system $D$ on $X$ satisfying $D^{2}=\rho_{*}^{-1}(D)$. Then, from the above information, we see that there exists a coframe $\left\{\varpi, \varpi_{1}, \ldots, \varpi_{n}, \omega_{r+1}, \ldots, \omega_{n}, \pi_{\alpha \beta}(r+1 \leqq\right.$ $\alpha \leqq \beta \leqq n)\}$ on a neighborhood of each $x \in X$ such that

$$
\begin{gathered}
D=\left\{\varpi=\varpi_{1}=\cdots=\varpi_{n}=0\right\}, \partial D=\left\{\varpi=\varpi_{1}=\cdots=\varpi_{r}=0\right\}, \\
\partial^{2} D=\partial^{(2)} D=\left\{\varpi_{1}=\cdots=\varpi_{r}=0\right\},
\end{gathered}
$$

and that

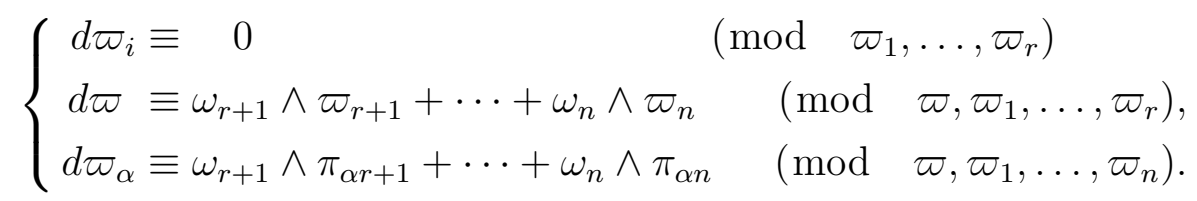

for $1 \leqq i \leqq r$ and $r+1 \leqq \alpha \leqq n$ and $\pi_{\alpha \beta}=\pi_{\beta \alpha}$. Thus $B=\partial^{2} D$ is completely integrable. Let $p_{1}, \ldots, p_{r}$ be the independent first integral of $B$ around $x \in X$. Then we obtain

$$
\begin{gathered}
D=\left\{\varpi=\varpi_{r+1}=\cdots=\varpi_{n}=d p_{1}=\cdots=d p_{r}=0\right\}, \\
\left\{\begin{array}{l}
d \varpi \equiv \omega_{r+1} \wedge \varpi_{r+1}+\cdots+\omega_{n} \wedge \varpi_{n} \quad\left(\bmod \varpi, d p_{1}, \ldots, d p_{r}\right), \\
d \varpi_{\alpha} \equiv \omega_{r+1} \wedge \pi_{\alpha r+1}+\cdots+\omega_{n} \wedge \pi_{\alpha n}\left(\bmod \varpi, \varpi_{r+1}, \ldots, \varpi_{n}, d p_{1}, \ldots, d p_{r}\right) .
\end{array}\right.
\end{gathered}
$$

for $r+1 \leqq \alpha \leqq n$.

Namely $(X, D)$ is a parametrized second order contact manifold. Hence, by the Darboux theorem, we obtain a coordinate system $\left(x_{\alpha}, z, p_{i}, p_{\alpha}, p_{\alpha \beta}\right)(1 \leqq i \leqq r, r+1 \leqq$ $\alpha, \leqq \beta \leqq n$ ) around $x \in X$ such that (see [Y4] $\S 4.2$ and [Y7] $\S 1.4$ )

$$
D=\left\{\hat{\varpi}=\hat{\varpi}_{r+1}=\cdots=\hat{\varpi}_{n}=d p_{1}=\cdots=d p_{r}=0\right\}
$$


where

$$
\hat{\varpi}=d z-\sum_{\alpha=r+1}^{n} p_{\alpha} d x_{\alpha}, \quad \hat{\varpi}_{\alpha}=d p_{\alpha}-\sum_{\beta=r+1}^{n} p_{\alpha \beta} d x_{\beta} .
$$

We refer the reader to $\S 4.2$ [Y4] for the detail to obtain a canonical coordinate system of $\left(R ; D^{1}, D^{2}\right)$.

Moreover we observe that $(X, D)$ satisfies three conditions in $\S 4.3$,

$$
R(X)=\left\{v \in P(X)\left|v \not \supset \partial^{2} D(x)\right| x=\nu(v)\right\} .
$$

and $\hat{\mathfrak{f}}(v)=\operatorname{Ch}(\partial D)(x)\left(\cong S^{2}\left(\left(V_{s}\right)^{*}\right)\right) \subset D(x)$ for $v \in R(X)$. Thus, by Proposition $4, R$ is involutive, because $S^{2}\left(\left(V_{s}\right)^{*}\right)$ is involutive.

6.2. $G_{2}$-Geometry. Let $\left(X_{\ell}, \Delta_{\theta}\right)$ be the (standard) contact gradation. Then we have $\Delta_{\theta}=\left\{\alpha_{\theta}\right\}$ except for $A_{\ell}$ type (see $\S 5.2$ ). As we observed in $\S 6.3$ in [Y6], for the exceptional simple Lie algebras, there exists, without exception, a unique simple root $\alpha_{G}$ next to $\alpha_{\theta}$ such that the coefficient of $\alpha_{G}$ in the highest root is 3 . We will consider simple graded Lie algebras $\left(X_{\ell},\left\{\alpha_{G}\right\}\right)$ of depth 3 and will show that regular differential systems of these types satisfy the conditions $(X .1)$ to $(X .3)$ in $\S 4.3$.

Explicitly we will here consider the following simple graded Lie algebras of depth 3: $\left(G_{2},\left\{\alpha_{1}\right\}\right),\left(F_{4},\left\{\alpha_{2}\right\}\right),\left(E_{6},\left\{\alpha_{4}\right\}\right),\left(E_{7},\left\{\alpha_{3}\right\}\right),\left(E_{8},\left\{\alpha_{7}\right\}\right),\left(B_{\ell},\left\{\alpha_{1}, \alpha_{3}\right\}\right)(\ell \geqq 3),\left(D_{\ell},\left\{\alpha_{1}, \alpha_{3}\right\}\right)$ $(\ell \geqq 5)$ and $\left(D_{4},\left\{\alpha_{1}, \alpha_{3}, \alpha_{4}\right\}\right)$. These graded Lie algebras have the common feature with $\left(G_{2},\left\{\alpha_{1}\right\}\right)$ as follows: In these cases, $\mathfrak{m}=\mathfrak{g}_{-3} \oplus \mathfrak{g}_{-2} \oplus \mathfrak{g}_{-1}$ satisfies $\operatorname{dim} \mathfrak{g}_{-3}=2$ and $\operatorname{dim} \mathfrak{g}_{-1}=2 \operatorname{dim} \mathfrak{g}_{-2}$. Moreover, in the description of the gradation in terms of the root space decomposition in $\S 5.1$, we have $\Phi_{3}^{+}=\left\{\theta, \theta-\alpha_{\theta}\right\}$ such that the coefficient of $\alpha_{\theta}$ in each $\beta \in \Phi_{2}^{+}$is 1 and $\Phi_{1}^{+}$consists of roots $\theta-\beta, \theta-\alpha_{\theta}-\beta$ for each $\beta \in \Phi_{2}^{+}$. Hence, ignoring the bracket product in $\mathfrak{g}_{-1}$, we can describe the bracket products of other part of $\mathfrak{m}$, in terms of paring, by

$$
\mathfrak{g}_{-3}=W, \quad \mathfrak{g}_{-2}=V \quad \text { and } \quad \mathfrak{g}_{-1}=W \otimes V^{*},
$$

where $\operatorname{dim} W=2$.

Thus let $(X, D)$ be a regular differential system of type $\mathfrak{m}$, where $\mathfrak{m}$ is the negative part of one of the above graded Lie algebras. Then $(X, \partial D)$ is a regular differential system of type $\mathfrak{c}^{1}(s, 2)$. Namely, there exists a coframe $\left\{\varpi_{1}, \varpi_{2}, \pi_{1}, \ldots, \pi_{s}, \pi_{1}^{1}, \ldots, \pi_{1}^{s}, \pi_{2}^{1}, \ldots, \pi_{2}^{s}\right\}$ around $x \in X$ such that

$$
\partial D=\left\{\varpi_{1}=\varpi_{2}=0\right\}
$$

and

$$
\left\{\begin{array}{lll}
d \varpi_{1} \equiv \pi_{1}^{1} \wedge \pi_{1}+\cdots+\pi_{1}^{s} \wedge \pi_{s} & (\bmod & \left.\varpi_{1}, \varpi_{2}\right) \\
d \varpi_{2} \equiv \pi_{2}^{1} \wedge \pi_{1}+\cdots+\pi_{2}^{s} \wedge \pi_{s} & (\bmod & \left.\varpi_{1}, \varpi_{2}\right)
\end{array}\right.
$$

Thus $(X, D)$ satisfies the conditions $(X .1)$ to $(X .3)$ in $\S 4.3$.

Now, putting $\varpi=\lambda_{1} \varpi_{1}+\lambda_{2} \varpi_{2}$, we consider a point $v \in P(X)$ such that $v=\{\varpi=$ $0\} \subset T_{x}(X)$, where $x=\nu(v)$. Then, for $\left(\lambda_{1}, \lambda_{2}\right) \neq(0,0),\left\{\lambda_{1} \pi_{1}^{i}+\lambda_{2} \pi_{2}^{i}(i=1, \ldots, s)\right\}$ are linearly independent $\left(\bmod \varpi_{1}, \varpi_{2}, \pi_{1}, \ldots, \pi_{s}\right)$. Thus $\hat{\mathfrak{f}}(v) \subset D(x)$ is of codimension $s$ at each $v \in P(X)$ (see the proof of Proposition 3). Hence we obtain $R(X)=P(X)$ in this case, i.e., $R(X)$ is a $\mathbb{P}^{1}$-bundle over $X$.

In fact, when $(X, D)$ is the model space $\left(M_{\mathfrak{g}}, D_{\mathfrak{g}}\right)$ of type $\left(X_{\ell},\left\{\alpha_{G}\right\}\right), R(X)$ can be identified with the model space $\left(R_{\mathfrak{g}}, E_{\mathfrak{g}}\right)$ of type $\left(X_{\ell},\left\{\alpha_{\theta}, \alpha_{G}\right\}\right)$ as follows (here, we understand $\alpha_{G}$ denotes two simple roots $\alpha_{1}$ and $\alpha_{3}$ in case of $B D_{\ell}$ types and three simple 
roots $\alpha_{1}, \alpha_{3}$ and $\alpha_{4}$ in case of $\left.D_{4}\right)$ : Let $\left(J_{\mathfrak{g}}, C_{\mathfrak{g}}\right)$ be the standard contact manifold of type $\left(X_{\ell},\left\{\alpha_{\theta}\right\}\right)$. Then we have the double fibration;

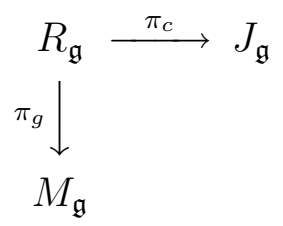

Here $\left(X_{\ell},\left\{\alpha_{\theta}, \alpha_{G}\right\}\right)$ is a graded Lie algebra of depth 5 and satisfies the following: $\operatorname{dim} \check{\mathfrak{g}}_{-5}=$ $\operatorname{dim} \check{\mathfrak{g}}_{-4}=1, \operatorname{dim} \check{\mathfrak{g}}_{-3}=\operatorname{dim} \check{\mathfrak{g}}_{-2}=s$ and $\operatorname{dim} \check{\mathfrak{g}}_{-1}=s+1$. In fact, comparing with the gradation of $\left(X_{\ell},\left\{\alpha_{G}\right\}\right)$, we have $\check{\Phi}_{5}^{+}=\{\theta\}, \check{\Phi}_{4}^{+}=\left\{\theta-\alpha_{\theta}\right\}, \check{\Phi}_{3}^{+}=\Phi_{2}^{+}, \check{\Phi}_{2}^{+}$consists of roots $\theta-\beta$ for each $\beta \in \check{\Phi}_{3}^{+}$and $\check{\Phi}_{1}^{+}$consists of roots $\alpha_{\theta}$ and $\theta-\alpha_{\theta}-\beta$ for each $\beta \in \check{\Phi}_{3}^{+}$. Thus we see that $\partial^{(3)} E_{\mathfrak{g}}=\left(\pi_{c}\right)_{*}^{-1}\left(C_{\mathfrak{g}}\right), \partial^{(2)} E_{\mathfrak{g}}=\left(\pi_{g}\right)_{*}^{-1}\left(\partial D_{\mathfrak{g}}\right)$ and $\partial E_{\mathfrak{g}}=\left(\pi_{g}\right)_{*}^{-1}\left(D_{g}\right)$. We put $D^{1}=\partial^{(3)} E_{\mathfrak{g}}$ and $D^{2}=\partial E_{\mathfrak{g}}$. Then $\left(R_{\mathfrak{g}} ; D^{1}, D^{2}\right)$ is a $P D$ manifold of second order. In fact, we have an isomorphism of $\left(R_{\mathfrak{g}} ; D^{1}, D^{2}\right)$ onto $\left(R\left(M_{\mathfrak{g}}\right) ; D_{M_{\mathfrak{g}}}^{1}, D_{M_{\mathfrak{g}}}^{2}\right)$ by the Realization Lemma for $\left(R_{\mathfrak{g}}, D^{1}, \pi_{g}, M_{\mathfrak{g}}\right)$ and an embedding of $R_{\mathfrak{g}}$ into $L\left(J_{\mathfrak{g}}\right)$ by the Realization Lemma for $\left(R_{\mathfrak{g}}, D^{2}, \pi_{c}, J_{\mathfrak{g}}\right)$. Thus $R_{\mathfrak{g}}$ is identified with a $R$-space orbit in $L\left(J_{\mathfrak{g}}\right)$.

Now we will calculate the symbol of $\left(R(X) ; D_{X}^{1}, D_{X}^{2}\right)$ by utilizing the model $P D$ manifold $\left(R_{\mathfrak{g}} ; D^{1}, D^{2}\right)$ of second order, especially when $\left(X_{\ell},\left\{\alpha_{\theta}, \alpha_{G}\right\}\right)$ is of $B D_{\ell}$ types. Let us describe the gradation of $\left(B D_{\ell},\left\{\alpha_{1}, \alpha_{2}, \alpha_{3}\right\}\right)$ or $\left(D_{4},\left\{\alpha_{1}, \alpha_{2}, \alpha_{3}, \alpha_{4}\right\}\right)$ in matrices form as follows: First we describe

$$
\mathfrak{o}(k+6)=\left\{X \in \mathfrak{g l}(k+6, \mathbb{K}) \mid{ }^{t} X J+J X=0\right\},
$$

where

$$
J=\left(\begin{array}{ccccccc}
0 & 0 & 0 & 0 & 0 & 0 & 1 \\
0 & 0 & 0 & 0 & 0 & 1 & 0 \\
0 & 0 & 0 & 0 & 1 & 0 & 0 \\
0 & 0 & 0 & I_{k} & 0 & 0 & 0 \\
0 & 0 & 1 & 0 & 0 & 0 & 0 \\
0 & 1 & 0 & 0 & 0 & 0 & 0 \\
1 & 0 & 0 & 0 & 0 & 0 & 0
\end{array}\right) \in \mathfrak{g l}(k+6, \mathbb{K}), \quad I_{k}=\left(\delta_{i j}\right) \in \mathfrak{g l}(k, \mathbb{K})
$$

Here $I_{k} \in \mathfrak{g l}(k, \mathbb{K})$ is the unit matrix and the gradation is given again by subdividing matrices as follows;

$$
\check{\mathfrak{g}}_{-5}=\left\{\left(\begin{array}{ccccccc}
0 & 0 & 0 & 0 & 0 & 0 & 0 \\
0 & 0 & 0 & 0 & 0 & 0 & 0 \\
0 & 0 & 0 & 0 & 0 & 0 & 0 \\
0 & 0 & 0 & 0 & 0 & 0 & 0 \\
0 & 0 & 0 & 0 & 0 & 0 & 0 \\
y & 0 & 0 & 0 & 0 & 0 & 0 \\
0 & -y & 0 & 0 & 0 & 0 & 0
\end{array}\right)\right\}, \check{\mathfrak{g}}_{-4}=\left\{\left(\begin{array}{ccccccc}
0 & 0 & 0 & 0 & 0 & 0 & 0 \\
0 & 0 & 0 & 0 & 0 & 0 & 0 \\
0 & 0 & 0 & 0 & 0 & 0 & 0 \\
0 & 0 & 0 & 0 & 0 & 0 & 0 \\
\xi_{0} & 0 & 0 & 0 & 0 & 0 & 0 \\
0 & 0 & 0 & 0 & 0 & 0 & 0 \\
0 & 0 & -\xi_{0} & 0 & 0 & 0 & 0
\end{array}\right)\right\}, y, \xi_{0} \in \mathbb{K}
$$




$$
\begin{aligned}
& \check{\mathfrak{g}}_{-3}=\left\{\left(\begin{array}{ccccccc}
0 & 0 & 0 & 0 & 0 & 0 & 0 \\
0 & 0 & 0 & 0 & 0 & 0 & 0 \\
0 & 0 & 0 & 0 & 0 & 0 & 0 \\
\xi & 0 & 0 & 0 & 0 & 0 & 0 \\
0 & \xi_{1} & 0 & 0 & 0 & 0 & 0 \\
0 & 0 & -\xi_{1} & 0 & 0 & 0 & 0 \\
0 & 0 & 0 & -{ }^{t} \xi & 0 & 0 & 0
\end{array}\right) \mid \xi \in \mathbb{K}^{k}, \xi_{1} \in \mathbb{K}\right\} \\
& \check{\mathfrak{g}}_{-2}=\left\{\left(\begin{array}{ccccccc}
0 & 0 & 0 & 0 & 0 & 0 & 0 \\
0 & 0 & 0 & 0 & 0 & 0 & 0 \\
x_{1} & 0 & 0 & 0 & 0 & 0 & 0 \\
0 & x & 0 & 0 & 0 & 0 & 0 \\
0 & 0 & 0 & 0 & 0 & 0 & 0 \\
0 & 0 & 0 & -{ }^{t} x & 0 & 0 & 0 \\
0 & 0 & 0 & 0 & -x_{1} & 0 & 0
\end{array}\right)=\hat{x}+\hat{x}_{1} \mid x \in \mathbb{K}^{k}, x_{1} \in \mathbb{K}\right\} \text {, } \\
& \check{\mathfrak{g}}_{-1}=\left\{\left(\begin{array}{ccccccc}
0 & 0 & 0 & 0 & 0 & 0 & 0 \\
a_{1} & 0 & 0 & 0 & 0 & 0 & 0 \\
0 & x_{0} & 0 & 0 & 0 & 0 & 0 \\
0 & 0 & a & 0 & 0 & 0 & 0 \\
0 & 0 & 0 & -{ }^{t} a & 0 & 0 & 0 \\
0 & 0 & 0 & 0 & -x_{0} & 0 & 0 \\
0 & 0 & 0 & 0 & 0 & -a_{1} & 0
\end{array}\right)=\hat{x}_{0}+\hat{a}_{1}+\hat{a} \mid \begin{array}{r}
x_{0}, a_{1} \in \mathbb{K}, \\
a \in \mathbb{K}^{k}
\end{array}\right\}, \\
& \check{\mathfrak{g}}_{0}=\left\{\left(\begin{array}{ccccccc}
b & 0 & 0 & 0 & 0 & 0 & 0 \\
0 & c & 0 & 0 & 0 & 0 & 0 \\
0 & 0 & e & 0 & 0 & 0 & 0 \\
0 & 0 & 0 & B & 0 & 0 & 0 \\
0 & 0 & 0 & 0 & -e & 0 & 0 \\
0 & 0 & 0 & 0 & 0 & -c & 0 \\
0 & 0 & 0 & 0 & 0 & 0 & -b
\end{array}\right) \mid b, c, e \in \mathbb{K}, B \in \mathfrak{o}(k)\right\} \\
& \check{\mathfrak{g}}_{\ell}=\left\{{ }^{t} X \mid X \in \mathfrak{g}_{-\ell}\right\},(\ell=1,2,3,4,5) \text {, }
\end{aligned}
$$

Then, for $X=\hat{x}+\hat{x}_{1}+\hat{x}_{0}$ and $A=\hat{a}+\hat{a}_{1}$, we calculate

$$
[[A, X], X]=\left(2 x_{1}{ }^{t} \widehat{a x-a_{1}}{ }^{t} x x\right) \in \check{\mathfrak{g}}_{-5}
$$

Thus we obtain

$$
\mathfrak{f}=\left\langle\left\{2 e_{1}^{*} \odot e_{2}^{*}, \ldots, 2 e_{1}^{*} \odot e_{k+1}^{*}, e_{2}^{*} \odot e_{2}^{*}+\cdots+e_{k+1}^{*} \odot e_{k+1}^{*}\right\rangle \subset S^{2}\left(E^{\perp}\right),\right.
$$

where $\left\{e_{0}, e_{1}, \ldots, e_{k+1}\right\}$ is a basis of $V$ and $E=\left\langle\left\{e_{0}\right\}\right\rangle$ is the Cauchy characteristic direction. In case $k=1, \mathfrak{f}=\left\langle\left\{2 e_{1}^{*} \odot e_{2}^{*}, e_{2}^{*} \odot e_{2}^{*}\right\}\right\rangle$ is an involutive subspace of $S^{2}\left(E^{\perp}\right)$. Hence $\left(R_{\mathfrak{g}} ; D^{1}, D^{2}\right)$ is involutive when $\mathfrak{g}=\bigoplus_{p \in \mathbb{Z}} \mathfrak{g}_{p}$ is of type $\left(B_{3},\left\{\alpha_{1}, \alpha_{2}, \alpha_{3}\right\}\right)$. In case $k>1$, we have

$$
\begin{aligned}
\mathfrak{f}^{\perp}=\left\langle\left\{ e_{1} \odot e_{1}, e_{i} \odot e_{j}(2 \leqq i<j\right.\right. & \leqq k+1), \\
& \left.\left.e_{2} \odot e_{2}-e_{k+1} \odot e_{k+1}, \ldots, e_{k} \odot e_{k}-e_{k+1} \odot e_{k+1}\right\}\right\rangle \subset S^{2}(W),
\end{aligned}
$$

where $W=\left\langle\left\{e_{1}, \ldots, e_{k+1}\right\}\right\rangle$. Then we see that $\left(\mathfrak{f}^{(1)}\right)^{\perp}$ contains every $e_{i} \odot e_{i} \odot e_{i}$ for $i=1, \ldots, k+1$, which implies $\mathfrak{f}$ is of finite type. We can also check that exceptional cases other than $G_{2}$ are of finite type by utilizing $R$-space orbit $\left(R_{\mathfrak{g}} ; D^{1}, D^{2}\right)$, whereas 
$\mathfrak{f}=S^{2}\left(W^{*}\right)$ is involutive in case of $G_{2}$, where $\operatorname{dim} W=1$. We will discuss these cases in a uniform way in other occasion.

6.3. Other Examples. We exhibit here two other examples of simple graded Lie algebras $\mathfrak{g}=\bigoplus_{p \in \mathbb{Z}} \mathfrak{g}_{p}$ of depth 3 , such that regular differential systems $(X, D)$ of type $\mathfrak{m}$ satisfy the conditions $(X .1)$ to $(X .3)$ in $\S 4.3$, where $\mathfrak{m}=\bigoplus_{p<0} \mathfrak{g}_{p}$.

The first example is of type $\left(C_{\ell},\left\{\alpha_{2}, \alpha_{\ell}\right\}\right)$. Here we have $\operatorname{dim} \mathfrak{g}_{-3}=3, \operatorname{dim} \mathfrak{g}_{-2}=2(\ell-2)$ and $\operatorname{dim} \mathfrak{g}_{-1}=2(\ell-2)+\frac{1}{2}(\ell-2)(\ell-1)$. Utilizing the calculation in Case $(3)$ of $\S 3$ in [YY2], we have the following description of the standard differential system $\left(M_{\mathfrak{m}}, D_{\mathfrak{m}}\right)$ of type $\mathfrak{m}$ in this case:

$$
D_{\mathfrak{m}}=\left\{\varpi_{0}=\varpi_{1}=\varpi_{2}=\pi_{1}^{1}=\cdots=\pi_{1}^{\ell-2}=\pi_{2}^{1}=\cdots=\pi_{2}^{\ell-2}=0\right\},
$$

where

$$
\left\{\begin{array}{l}
\varpi_{0}=d y_{0}-\sum_{\alpha=1}^{\ell-2} \xi_{1}^{\alpha} d x_{2}^{\alpha}-\sum_{\alpha=1}^{\ell-2} \xi_{2}^{\alpha} d x_{1}^{\alpha}, \\
\varpi_{1}=d y_{1}-2 \sum_{\alpha=1}^{\ell-2} \xi_{1}^{\alpha} d x_{1}^{\alpha}, \quad \varpi_{2}=d y_{2}-2 \sum_{\alpha=1}^{\ell-2} \xi_{2}^{\alpha} d x_{2}^{\alpha}, \\
\pi_{p}^{\alpha}=d \xi_{p}^{\alpha}-\sum_{\beta=1}^{\ell-2} a_{\alpha \beta} d x_{p}^{\beta} \quad(p=1,2 \quad \alpha=1, \ldots, \ell-2),
\end{array}\right.
$$

Here we put $y_{12}=y_{0}, y_{11}=y_{1}, y_{22}=y_{2}$ and $a_{\alpha \beta}=a_{\beta \alpha}$ for $1 \leqq \alpha, \beta \leqq \ell-2$. Let $(X, D)$ be a regular differential system of type $\mathfrak{m}$. Then we have the structure equation of $(X, \partial D)$ as follows;

$$
\left\{\begin{array}{l}
d \varpi_{0} \equiv \omega_{2}^{1} \wedge \pi_{1}^{1}+\cdots+\omega_{2}^{\ell-2} \wedge \pi_{1}^{\ell-2}+\omega_{1}^{1} \wedge \pi_{2}^{1}+\cdots+\omega_{1}^{\ell-2} \wedge \pi_{2}^{\ell-2} \\
d \varpi_{1} \equiv 2 \omega_{1}^{1} \wedge \pi_{1}^{1}+\cdots+2 \omega_{1}^{\ell-2} \wedge \pi_{1}^{\ell-2} \quad\left(\bmod \quad \varpi_{0}, \varpi_{1}, \varpi_{2}\right) \\
d \varpi_{2} \equiv 2 \omega_{2}^{1} \wedge \pi_{2}^{1}+\cdots+2 \omega_{2}^{\ell-2} \wedge \pi_{2}^{\ell-2}
\end{array}\right.
$$

Now, putting $\varpi=\lambda_{0} \varpi_{0}+\lambda_{1} \varpi_{1}+\lambda_{2} \varpi_{2}$, we consider a point $v \in P(X)$ such that $v=\{\varpi=0\} \subset T_{x}(X)$, where $x=\nu(v)$. Then, from

$$
d \varpi \equiv \sum_{\alpha=1}^{\ell-2}\left(\lambda_{0} \omega_{2}^{\alpha}+2 \lambda_{1} \omega_{1}^{\alpha}\right) \wedge \pi_{1}^{\alpha}+\sum_{\alpha=1}^{\ell-2}\left(\lambda_{0} \omega_{1}^{\alpha}+2 \lambda_{2} \omega_{2}^{\alpha}\right) \wedge \pi_{2}^{\alpha}, \quad\left(\bmod \varpi_{0}, \varpi_{1}, \varpi_{2}\right),
$$

and

$$
\left(\lambda_{0} \omega_{2}^{\alpha}+2 \lambda_{1} \omega_{1}^{\alpha}\right) \wedge\left(\lambda_{0} \omega_{1}^{\alpha}+2 \lambda_{2} \omega_{2}^{\alpha}\right)=\left(4 \lambda_{1} \lambda_{2}-\lambda_{0}^{2}\right) \omega_{1}^{\alpha} \wedge \omega_{2}^{\alpha},
$$

we see that $(X, D)$ satifies the condition $(X .1)$ to $(X .3)$ in $\S 4.3$ and we obtain

$$
R(X)=\left\{v \in P(X) \mid 4 \lambda_{1} \lambda_{2}-\lambda_{0}^{2} \neq 0\right\},
$$

where $\left(\lambda_{0}, \lambda_{1}, \lambda_{2}\right)$ is the homogeneous coordinate of the fibre $\nu: P(X) \rightarrow X$.

By the calculation in Case (3) of $\S 3$ in [YY2], we have $y_{p q}=2 \sum_{\alpha, \beta=1}^{\ell-2} a_{\alpha \beta} x_{p}^{\alpha} x_{q}^{\beta}$ for $1 \leqq p \leqq q \leqq 2$ and $a_{\alpha \beta}=a_{\beta \alpha}(1 \leqq \alpha, \beta \leqq \ell-2)$ so that

$$
\lambda_{0} y_{0}+\lambda_{1} y_{1}+\lambda_{2} y_{2}=2 \sum_{\alpha, \beta=1}^{\ell-2} a_{\alpha \beta}\left(\lambda_{0} x_{1}^{\alpha} x_{2}^{\beta}+\lambda_{1} x_{1}^{\alpha} x_{1}^{\beta}+\lambda_{2} x_{2}^{\alpha} x_{2}^{\beta}\right) .
$$


Hence, from

$$
\begin{aligned}
\sum_{\alpha, \beta=1}^{\ell-2} a_{\alpha \beta}\left(\lambda_{0} x_{1}^{\alpha} x_{2}^{\beta}+\lambda_{1} x_{1}^{\alpha} x_{1}^{\beta}+\lambda_{2} x_{2}^{\alpha} x_{2}^{\beta}\right) & =\sum_{\alpha=1}^{\ell-2} a_{\alpha \alpha}\left(\lambda_{0} x_{1}^{\alpha} x_{2}^{\alpha}+\lambda_{1} x_{1}^{\alpha} x_{1}^{\alpha}+\lambda_{2} x_{2}^{\alpha} x_{2}^{\alpha}\right) \\
+ & \sum_{\alpha<\beta} a_{\alpha \beta}\left(\lambda_{0}\left(x_{1}^{\alpha} x_{2}^{\beta}+x_{1}^{\beta} x_{2}^{\alpha}\right)+2 \lambda_{1} x_{1}^{\alpha} x_{1}^{\beta}+2 \lambda_{2} x_{2}^{\alpha} x_{2}^{\beta}\right),
\end{aligned}
$$

we have

$$
\begin{gathered}
\hat{\mathfrak{f}}(v)=\left\langle\left\{\lambda_{0}\left(e_{1}^{\alpha}\right)^{*} \odot\left(e_{2}^{\alpha}\right)^{*}+\lambda_{1}\left(e_{1}^{\alpha}\right)^{*} \odot\left(e_{1}^{\alpha}\right)^{*}+\lambda_{2}\left(e_{2}^{\alpha}\right)^{*} \odot\left(e_{2}^{\alpha}\right)^{*}(1 \leqq \alpha \leqq \ell-2),\right.\right. \\
\lambda_{0}\left(\left(e_{1}^{\alpha}\right)^{*} \odot\left(e_{2}^{\beta}\right)^{*}+\left(e_{1}^{\beta}\right)^{*} \odot\left(e_{2}^{\alpha}\right)^{*}\right)+2 \lambda_{1}\left(e_{1}^{\alpha}\right)^{*} \odot\left(e_{1}^{\beta}\right)^{*}+2 \lambda_{2}\left(e_{2}^{\alpha}\right)^{*} \odot\left(e_{2}^{\beta}\right)^{*} \\
(1 \leqq \alpha<\beta \leqq \ell-2)\}\rangle \subset S^{2}\left(W^{*}\right),
\end{gathered}
$$

where $W=\left\langle\left\{e_{1}^{1}, \ldots, e_{1}^{\ell-2}, e_{2}^{1}, \ldots, e_{2}^{\ell-2}\right\}\right\rangle$. Thus, assuming $\lambda_{0} \neq 0$, we get

$$
\begin{gathered}
\hat{\mathfrak{f}}(v)^{\perp}=\left\langle\left\{\lambda_{0} e_{1}^{\alpha} \odot e_{1}^{\alpha}-2 \lambda_{1} e_{1}^{\alpha} \odot e_{2}^{\alpha}, \lambda_{0} e_{2}^{\alpha} \odot e_{2}^{\alpha}-2 \lambda_{2} e_{1}^{\alpha} \odot e_{2}^{\alpha}(1 \leqq \alpha \leqq \ell-2),\right.\right. \\
e_{1}^{\alpha} \odot e_{2}^{\beta}-e_{1}^{\beta} \odot e_{2}^{\alpha}, \lambda_{0} e_{1}^{\alpha} \odot e_{1}^{\beta}-2 \lambda_{1} e_{1}^{\alpha} \odot e_{2}^{\beta}, \lambda_{0} e_{2}^{\alpha} \odot e_{2}^{\beta}-2 \lambda_{2} e_{1}^{\alpha} \odot e_{2}^{\beta}, \\
(1 \leqq \alpha<\beta \leqq \ell-2)\}\rangle \subset S^{2}(W),
\end{gathered}
$$

Then, from the first two generator of $\hat{\mathfrak{f}}(v)^{\perp}$, we see that $\hat{\mathfrak{f}}^{(1)}(v)^{\perp}$ contains $e_{1}^{\alpha} \odot e_{1}^{\alpha} \odot e_{2}^{\alpha}$ and $e_{1}^{\alpha} \odot e_{2}^{\alpha} \odot e_{2}^{\alpha}$. Moreover it follows that $\hat{\mathfrak{f}}^{(1)}(v)^{\perp}$ contains every $e_{1}^{\alpha} \odot e_{1}^{\alpha} \odot e_{1}^{\alpha}$ and $e_{2}^{\alpha} \odot e_{2}^{\alpha} \odot e_{2}^{\alpha}$ for $\alpha=1, \ldots, \ell-2$, which implies that $\hat{\mathfrak{f}}(v)$ is of finite type.

Now let us construct the model equation of second order from the coordinate description of the standard differential system $\left(M_{\mathfrak{m}}, D_{\mathfrak{m}}\right)$. We calculate

$$
\begin{aligned}
& \varpi=\varpi_{0}+\lambda_{1} \varpi_{1}+\lambda_{2} \varpi_{2} \\
&= d y_{0}+\lambda_{1} d y_{1}+\lambda_{2} d y_{2}-\sum_{\alpha=1}^{\ell-2}\left(\xi_{2}^{\alpha}+2 \lambda_{1} \xi_{1}^{\alpha}\right) d x_{1}^{\alpha}-\sum_{\alpha=1}^{\ell-2}\left(\xi_{1}^{\alpha}+2 \lambda_{2} \xi_{2}^{\alpha}\right) d x_{2}^{\alpha} \\
&=d\left(y_{0}+\lambda_{1} y_{1}+\lambda_{2} y_{2}\right)-y_{1} d \lambda_{1}-y_{2} d \lambda_{2} \\
& \quad-\sum_{\alpha=1}^{\ell-2}\left(\xi_{2}^{\alpha}+2 \lambda_{1} \xi_{1}^{\alpha}\right) d x_{1}^{\alpha}-\sum_{\alpha=1}^{\ell-2}\left(\xi_{1}^{\alpha}+2 \lambda_{2} \xi_{2}^{\alpha}\right) d x_{2}^{\alpha}
\end{aligned}
$$

Thus we put

$$
\left\{\begin{aligned}
z & =y_{0}+\lambda_{1} y_{1}+\lambda_{2} y_{2}, \quad x_{1}^{0}=\lambda_{1}, \quad x_{2}^{0}=\lambda_{2}, \quad p_{1}^{0}=y_{1}, \quad p_{2}^{0}=y_{2}, \\
p_{1}^{\alpha} & =\xi_{2}^{\alpha}+2 \lambda_{1} \xi_{1}^{\alpha}, \quad p_{2}^{\alpha}=\xi_{1}^{\alpha}+2 \lambda_{2} \xi_{2}^{\alpha} \quad(\alpha=1, \ldots, \ell-2) .
\end{aligned}\right.
$$

Then we have

$$
\xi_{1}^{\alpha}=\frac{p_{2}^{\alpha}-2 x_{2}^{0} p_{1}^{\alpha}}{1-4 x_{1}^{0} x_{2}^{0}}, \quad \xi_{2}^{\alpha}=\frac{p_{1}^{\alpha}-2 x_{1}^{0} p_{2}^{\alpha}}{1-4 x_{1}^{0} x_{2}^{0}}
$$

and

$$
\begin{aligned}
& \varpi=d z-\sum_{\alpha=0}^{\ell-2} p_{1}^{\alpha} d x_{1}^{\alpha}-\sum_{\alpha=0}^{\ell-2} p_{2}^{\alpha} d x_{2}^{\alpha}, \\
& \pi_{1}^{0}=d p_{1}^{0}-2 \sum_{\alpha=1}^{\ell-2} \xi_{1}^{\alpha} d x_{1}^{\alpha}, \quad \pi_{2}^{0}=d p_{2}^{0}-2 \sum_{\alpha=1}^{\ell-2} \xi_{2}^{\alpha} d x_{2}^{\alpha},
\end{aligned}
$$




$$
\begin{aligned}
& \pi_{1}^{\alpha}=d p_{1}^{\alpha}-2 \xi_{1}^{\alpha} d x_{1}^{0}-2 \sum_{\beta=1}^{\ell-2} \lambda_{1} a_{\alpha \beta} d x_{1}^{\beta}-\sum_{\beta=1}^{\ell-2} a_{\alpha \beta} d x_{2}^{\beta}, \\
& \pi_{2}^{\alpha}=d p_{2}^{\alpha}-2 \xi_{2}^{\alpha} d x_{2}^{0}-\sum_{\beta=1}^{\ell-2} a_{\alpha \beta} d x_{1}^{\beta}-2 \sum_{\beta=1}^{\ell-2} \lambda_{2} a_{\alpha \beta} d x_{2}^{\beta},
\end{aligned}
$$

where $\pi_{1}^{0}=\varpi_{1}, \pi_{2}^{0}=\varpi_{2}, \pi_{1}^{\alpha}=\varpi_{2}^{\alpha}+2 \lambda_{1} \varpi_{1}^{\alpha}$ and $\pi_{2}^{\alpha}=\varpi_{1}^{\alpha}+2 \lambda_{2} \varpi_{2}^{\alpha}$. Hence we obtain the following model equation of second order :

$$
\begin{array}{rlrl}
\frac{\partial^{2} z}{\partial x_{1}^{0} \partial x_{1}^{0}}=\frac{\partial^{2} z}{\partial x_{2}^{0} \partial x_{2}^{0}}=\frac{\partial^{2} z}{\partial x_{1}^{0} \partial x_{2}^{0}}=0, & \frac{\partial^{2} z}{\partial x_{1}^{0} \partial x_{2}^{\alpha}}=\frac{\partial^{2} z}{\partial x_{2}^{0} \partial x_{1}^{\alpha}}=0, \\
\frac{\partial^{2} z}{\partial x_{1}^{0} \partial x_{1}^{\alpha}}=\frac{2}{1-4 x_{1}^{0} x_{2}^{0}}\left(\frac{\partial z}{\partial x_{2}^{\alpha}}-2 x_{2}^{0} \frac{\partial z}{\partial x_{1}^{\alpha}}\right) \quad\left(=2 \xi_{1}^{\alpha}\right), & \\
\frac{\partial^{2} z}{\partial x_{2}^{0} \partial x_{2}^{\alpha}}=\frac{2}{1-4 x_{1}^{0} x_{2}^{0}}\left(\frac{\partial z}{\partial x_{1}^{\alpha}}-2 x_{1}^{0} \frac{\partial z}{\partial x_{2}^{\alpha}}\right) & \left(=2 \xi_{2}^{\alpha}\right), \\
\frac{\partial^{2} z}{\partial x_{1}^{\alpha} \partial x_{1}^{\beta}}=2 x_{1}^{0} \frac{\partial^{2} z}{\partial x_{2}^{\alpha} \partial x_{1}^{\beta}}, \quad \frac{\partial^{2} z}{\partial x_{2}^{\alpha} \partial x_{2}^{\beta}}=2 x_{2}^{0} \frac{\partial^{2} z}{\partial x_{2}^{\alpha} \partial x_{1}^{\beta}}, \\
\frac{\partial^{2} z}{\partial x_{2}^{\alpha} \partial x_{1}^{\beta}}=\frac{\partial^{2} z}{\partial x_{2}^{\beta} \partial x_{1}^{\alpha}} \quad\left(=a_{\alpha \beta}\right) & (1 \leqq \alpha \leqq \beta \leqq \ell-2) .
\end{array}
$$

Our second example is of type $\left(E_{7},\left\{\alpha_{6}, \alpha_{7}\right\}\right)$. Here we have $\operatorname{dim} \mathfrak{g}_{-3}=10, \operatorname{dim} \mathfrak{g}_{-2}=16$ and $\operatorname{dim} \mathfrak{g}_{-1}=16+1$. Utilizing the calculation in Case (4) of $\S 4$ in [YY2], we have the following description of the standard differential system $\left(M_{\mathfrak{m}}, D_{\mathfrak{m}}\right)$ of type $\mathfrak{m}$ in this case:

$$
D_{\mathfrak{m}}=\left\{\varpi_{1}=\cdots=\varpi_{10}=\pi_{1}=\cdots=\pi_{16}=0\right\},
$$

where

$$
\begin{aligned}
\varpi_{1} & =d y_{1}-p_{11} d x_{1}+p_{9} d x_{2}-p_{6} d x_{3}+p_{5} d x_{4}+p_{4} d x_{5}-p_{3} d x_{6}+p_{2} d x_{9}-p_{1} d x_{11}, \\
\varpi_{2} & =d y_{2}-p_{13} d x_{1}+p_{10} d x_{2}-p_{8} d x_{3}+p_{7} d x_{4}+p_{4} d x_{7}-p_{3} d x_{8}+p_{2} d x_{10}-p_{1} d x_{13}, \\
\varpi_{3} & =d y_{3}-p_{14} d x_{1}+p_{12} d x_{2}-p_{8} d x_{5}+p_{7} d x_{6}+p_{6} d x_{7}-p_{5} d x_{8}+p_{2} d x_{12}-p_{1} d x_{14}, \\
\varpi_{4} & =d y_{4}-p_{15} d x_{1}+p_{12} d x_{3}-p_{10} d x_{5}+p_{9} d x_{7}+p_{7} d x_{9}-p_{5} d x_{10}+p_{3} d x_{12}-p_{1} d x_{15}, \\
\varpi_{5} & =d y_{5}-p_{15} d x_{2}+p_{14} d x_{3}-p_{13} d x_{5}+p_{11} d x_{7}+p_{7} d x_{11}-p_{5} d x_{13}+p_{3} d x_{14}-p_{2} d x_{15}, \\
\varpi_{6} & =d y_{6}-p_{16} d x_{1}+p_{12} d x_{4}-p_{10} d x_{6}+p_{9} d x_{8}+p_{8} d x_{9}-p_{6} d x_{10}+p_{4} d x_{12}-p_{1} d x_{16}, \\
\varpi_{7} & =d y_{7}-p_{16} d x_{2}+p_{14} d x_{4}-p_{13} d x_{6}+p_{11} d x_{8}+p_{8} d x_{11}-p_{6} d x_{13}+p_{4} d x_{14}-p_{2} d x_{16}, \\
\varpi_{8} & =d y_{8}-p_{16} d x_{3}+p_{15} d x_{4}-p_{13} d x_{9}+p_{11} d x_{10}+p_{10} d x_{11}-p_{9} d x_{13}+p_{4} d x_{15}-p_{3} d x_{16}, \\
\varpi_{9} & =d y_{9}-p_{16} d x_{5}+p_{15} d x_{6}-p_{14} d x_{9}+p_{12} d x_{11}+p_{11} d x_{12}-p_{9} d x_{14}+p_{6} d x_{15}-p_{5} d x_{16}, \\
\varpi_{10} & =d y_{10}-p_{16} d x_{7}+p_{15} d x_{8}-p_{14} d x_{10}+p_{13} d x_{12}+p_{12} d x_{13}-p_{10} d x_{14}+p_{8} d x_{15}-p_{7} d x_{16}, \\
\pi_{i} & =d p_{i}-a d x_{i} \quad(i=1,2, \ldots, 16) .
\end{aligned}
$$


Let $(X, D)$ be a regular differential system of type $\mathfrak{m}$. Then we have the structure equation of $(X, \partial D)$ as follows;

$$
\begin{aligned}
d \varpi_{1} & \equiv \omega_{1} \wedge \pi_{11}-\omega_{2} \wedge \pi_{9}+\omega_{3} \wedge \pi_{6}-\omega_{4} \wedge \pi_{5}-\omega_{5} \wedge \pi_{4}+\omega_{6} \wedge \pi_{3}-\omega_{9} \wedge \pi_{2}+\omega_{11} \wedge \pi_{1}, \\
d \varpi_{2} & \equiv \omega_{1} \wedge \pi_{13}-\omega_{2} \wedge \pi_{10}+\omega_{3} \wedge \pi_{8}-\omega_{4} \wedge \pi_{7}-\omega_{7} \wedge \pi_{4}+\omega_{8} \wedge \pi_{3}-\omega_{10} \wedge \pi_{2}+\omega_{13} \wedge \pi_{1}, \\
d \varpi_{3} & \equiv \omega_{1} \wedge \pi_{14}-\omega_{2} \wedge \pi_{12}+\omega_{5} \wedge \pi_{8}-\omega_{6} \wedge \pi_{7}-\omega_{7} \wedge \pi_{6}+\omega_{8} \wedge \pi_{5}-\omega_{12} \wedge \pi_{2}+\omega_{14} \wedge \pi_{1}, \\
d \varpi_{4} & \equiv \omega_{1} \wedge \pi_{15}-\omega_{3} \wedge \pi_{12}+\omega_{5} \wedge \pi_{10}-\omega_{7} \wedge \pi_{9}-\omega_{9} \wedge \pi_{7}+\omega_{10} \wedge \pi_{5}-\omega_{12} \wedge \pi_{3}+\omega_{15} \wedge \pi_{1}, \\
d \varpi_{5} & \equiv \omega_{2} \wedge \pi_{15}-\omega_{3} \wedge \pi_{14}+\omega_{5} \wedge \pi_{13}-\omega_{7} \wedge \pi_{11}-\omega_{11} \wedge \pi_{7}+\omega_{13} \wedge \pi_{5}-\omega_{14} \wedge \pi_{3}+\omega_{15} \wedge \pi_{2}, \\
d \varpi_{6} & \equiv \omega_{1} \wedge \pi_{16}-\omega_{4} \wedge \pi_{12}+\omega_{6} \wedge \pi_{10}-\omega_{8} \wedge \pi_{9}-\omega_{9} \wedge \pi_{8}+\omega_{10} \wedge \pi_{6}-\omega_{12} \wedge \pi_{4}+\omega_{16} \wedge \pi_{1}, \\
d \varpi_{7} & \equiv \omega_{2} \wedge \pi_{16}-\omega_{4} \wedge \pi_{14}+\omega_{6} \wedge \pi_{13}-\omega_{8} \wedge \pi_{11}-\omega_{11} \wedge \pi_{8}+\omega_{13} \wedge \pi_{6}-\omega_{14} \wedge \pi_{4}+\omega_{16} \wedge \pi_{2}, \\
d \varpi_{8} & \equiv \omega_{3} \wedge \pi_{16}-\omega_{4} \wedge \pi_{15}+\omega_{9} \wedge \pi_{13}-\omega_{10} \wedge \pi_{11}-\omega_{11} \wedge \pi_{10}+\omega_{13} \wedge \pi_{9}-\omega_{15} \wedge \pi_{4}+\omega_{16} \wedge \pi_{3}, \\
d \varpi_{9} & \equiv \omega_{5} \wedge \pi_{16}-\omega_{6} \wedge \pi_{15}+\omega_{9} \wedge \pi_{14}-\omega_{11} \wedge \pi_{12}-\omega_{12} \wedge \pi_{11}+\omega_{14} \wedge \pi_{9}-\omega_{15} \wedge \pi_{6}+\omega_{16} \wedge \pi_{5}, \\
d \varpi_{10} & \equiv \omega_{7} \wedge \pi_{16}-\omega_{8} \wedge \pi_{15}+\omega_{10} \wedge \pi_{14}-\omega_{12} \wedge \pi_{13}-\omega_{13} \wedge \pi_{12}+\omega_{14} \wedge \pi_{10}-\omega_{15} \wedge \pi_{8}+\omega_{16} \wedge \pi_{7}, \\
\left(\bmod \quad \varpi_{1}, \ldots, \varpi_{10}\right) . &
\end{aligned}
$$

Now, putting $\varpi=\varpi_{1}+\sum_{i=2}^{10} \lambda_{i} \varpi_{i}$, we consider a point $v \in P(X)$ such that $v=\{\varpi=$ $0\} \subset T_{x}(X)$, where $x=\nu(v)$. Then we have

$$
d \varpi \equiv \check{\omega}_{1} \wedge \pi_{1}+\cdots+\check{\omega}_{16} \wedge \pi_{16} \quad\left(\bmod \quad \varpi_{1}, \ldots, \varpi_{10}\right),
$$

where

$$
\begin{array}{ll}
\check{\omega}_{1}=\omega_{11}+\lambda_{2} \omega_{13}+\lambda_{3} \omega_{14}+\lambda_{4} \omega_{15}+\lambda_{6} \omega_{16}, & \check{\omega}_{2}=-\omega_{9}-\lambda_{2} \omega_{10}-\lambda_{3} \omega_{12}+\lambda_{5} \omega_{15}+\lambda_{7} \omega_{16}, \\
\check{\omega}_{3}=\omega_{6}+\lambda_{2} \omega_{8}-\lambda_{4} \omega_{12}-\lambda_{5} \omega_{14}+\lambda_{8} \omega_{16}, & \check{\omega}_{4}=-\omega_{5}-\lambda_{2} \omega_{7}-\lambda_{6} \omega_{12}-\lambda_{7} \omega_{14}-\lambda_{8} \omega_{15}, \\
\check{\omega}_{5}=-\omega_{4}+\lambda_{3} \omega_{8}+\lambda_{4} \omega_{10}+\lambda_{5} \omega_{13}+\lambda_{9} \omega_{16}, & \check{\omega}_{6}=\omega_{3}-\lambda_{3} \omega_{7}+\lambda_{6} \omega_{10}+\lambda_{7} \omega_{13}-\lambda_{9} \omega_{15}, \\
\check{\omega}_{7}=-\lambda_{2} \omega_{4}-\lambda_{3} \omega_{6}-\lambda_{4} \omega_{9}-\lambda_{5} \omega_{11}+\lambda_{10} \omega_{16}, & \check{\omega}_{8}=\lambda_{2} \omega_{3}+\lambda_{3} \omega_{5}-\lambda_{6} \omega_{9}-\lambda_{7} \omega_{11}-\lambda_{10} \omega_{15}, \\
\check{\omega}_{9}=-\omega_{2}-\lambda_{4} \omega_{7}-\lambda_{6} \omega_{8}+\lambda_{8} \omega_{13}+\lambda_{9} \omega_{14}, & \check{\omega}_{10}=-\lambda_{2} \omega_{2}+\lambda_{4} \omega_{5}+\lambda_{6} \omega_{6}-\lambda_{8} \omega_{11}+\lambda_{10} \omega_{14}, \\
\check{\omega}_{11}=\omega_{1}-\lambda_{5} \omega_{7}-\lambda_{7} \omega_{8}-\lambda_{8} \omega_{10}-\lambda_{9} \omega_{12}, & \check{\omega}_{12}=-\lambda_{3} \omega_{2}-\lambda_{4} \omega_{3}-\lambda_{6} \omega_{4}-\lambda_{9} \omega_{11}-\lambda_{10} \omega_{13}, \\
\check{\omega}_{13}=\lambda_{2} \omega_{1}+\lambda_{5} \omega_{5}+\lambda_{7} \omega_{6}+\lambda_{8} \omega_{9}-\lambda_{10} \omega_{12}, & \check{\omega}_{14}=\lambda_{3} \omega_{1}-\lambda_{5} \omega_{3}-\lambda_{7} \omega_{4}+\lambda_{9} \omega_{9}+\lambda_{10} \omega_{10}, \\
\check{\omega}_{15}=\lambda_{4} \omega_{1}+\lambda_{5} \omega_{2}-\lambda_{8} \omega_{4}-\lambda_{9} \omega_{6}-\lambda_{10} \omega_{8}, & \check{\omega}_{16}=\lambda_{6} \omega_{1}+\lambda_{7} \omega_{2}+\lambda_{8} \omega_{3}+\lambda_{9} \omega_{5}+\lambda_{10} \omega_{7} .
\end{array}
$$

We calculate

$$
\begin{array}{ll}
\check{\omega}_{7}=-\lambda_{5} \check{\omega}_{1}+\lambda_{4} \check{\omega}_{2}-\lambda_{3} \check{\omega}_{3}+\lambda_{2} \check{\omega}_{5}+\lambda \omega_{16}, & \check{\omega}_{8}=-\lambda_{7} \check{\omega}_{1}+\lambda_{6} \check{\omega}_{2}-\lambda_{3} \check{\omega}_{4}+\lambda_{2} \check{\omega}_{6}-\lambda \omega_{15}, \\
\check{\omega}_{10}=-\lambda_{8} \check{\omega}_{1}+\lambda_{6} \check{\omega}_{3}-\lambda_{4} \check{\omega}_{4}+\lambda_{2} \check{\omega}_{9}+\lambda \omega_{14}, & \check{\omega}_{12}=-\lambda_{9} \check{\omega}_{1}+\lambda_{6} \check{\omega}_{5}-\lambda_{4} \check{\omega}_{6}+\lambda_{3} \check{\omega}_{9}-\lambda \omega_{13}, \\
\check{\omega}_{13}=-\lambda_{8} \check{\omega}_{2}+\lambda_{7} \check{\omega}_{3}-\lambda_{5} \check{\omega}_{4}+\lambda_{2} \check{\omega}_{11}-\lambda \omega_{12}, & \check{\omega}_{14}=-\lambda_{9} \check{\omega}_{2}+\lambda_{7} \check{\omega}_{5}-\lambda_{5} \check{\omega}_{6}+\lambda_{3} \check{\omega}_{11}+\lambda \omega_{10}, \\
\check{\omega}_{15}=-\lambda_{9} \check{\omega}_{3}+\lambda_{8} \check{\omega}_{5}-\lambda_{5} \check{\omega}_{9}+\lambda_{4} \check{\omega}_{11}-\lambda \omega_{8}, & \check{\omega}_{16}=-\lambda_{9} \check{\omega}_{4}+\lambda_{8} \check{\omega}_{6}-\lambda_{7} \check{\omega}_{9}+\lambda_{6} \check{\omega}_{11}+\lambda \omega_{7},
\end{array}
$$

where $\lambda=\lambda_{10}-\lambda_{2} \lambda_{9}+\lambda_{3} \lambda_{8}-\lambda_{4} \lambda_{7}+\lambda_{5} \lambda_{6}$. Thus we see that $(X, D)$ satifies the condition $(X .1)$ to $(X .3)$ in $\S 4.3$ and we obtain

$$
R(X)=\{v \in P(X) \mid \lambda \neq 0\}
$$

where $\left(\lambda_{2}, \ldots, \lambda_{10}\right)$ is the inhomogeneous coordinate of the fibre $\nu: P(X) \rightarrow X$. Moreover we have

$$
\hat{\mathfrak{f}}(v)=\left\{X \in \mathfrak{g}_{-1}(x) \mid\left[X, \mathfrak{g}_{-2}(x)\right]=0\right\} \quad \text { for each } v \in R(X), x=\nu(v) .
$$

and $\operatorname{dim} \hat{\mathfrak{f}}(v)=1$. Utilizing the calculation in Case (4) of $\S 4$ in [YY2], we have $f=\left(e_{1}^{*} \odot e_{11}^{*}-e_{2}^{*} \odot e_{9}^{*}+e_{3}^{*} \odot e_{6}^{*}-e_{4}^{*} \odot e_{5}^{*}\right)+\lambda_{2}\left(e_{1}^{*} \odot e_{13}^{*}-e_{2}^{*} \odot e_{10}^{*}+e_{3}^{*} \odot e_{8}^{*}-e_{4}^{*} \odot e_{7}^{*}\right)$ 


$$
\begin{aligned}
& +\lambda_{3}\left(e_{1}^{*} \odot e_{14}^{*}-e_{2}^{*} \odot e_{12}^{*}+e_{5}^{*} \odot e_{8}^{*}-e_{6}^{*} \odot e_{7}^{*}\right)+\lambda_{4}\left(e_{1}^{*} \odot e_{15}^{*}-e_{3}^{*} \odot e_{12}^{*}+e_{5}^{*} \odot e_{10}^{*}-e_{7}^{*} \odot e_{9}^{*}\right) \\
& +\lambda_{5}\left(e_{2}^{*} \odot e_{15}^{*}-e_{3}^{*} \odot e_{14}^{*}+e_{5}^{*} \odot e_{13}^{*}-e_{7}^{*} \odot e_{11}^{*}\right)+\lambda_{6}\left(e_{1}^{*} \odot e_{16}^{*}-e_{4}^{*} \odot e_{12}^{*}+e_{6}^{*} \odot e_{10}^{*}-e_{8}^{*} \odot e_{9}^{*}\right) \\
& +\lambda_{7}\left(e_{2}^{*} \odot e_{16}^{*}-e_{4}^{*} \odot e_{14}^{*}+e_{6}^{*} \odot e_{13}^{*}-e_{8}^{*} \odot e_{11}^{*}\right)+\lambda_{8}\left(e_{3}^{*} \odot e_{16}^{*}-e_{4}^{*} \odot e_{15}^{*}+e_{9}^{*} \odot e_{13}^{*}-e_{10}^{*} \odot e_{11}^{*}\right) \\
& +\lambda_{9}\left(e_{5}^{*} \odot e_{16}^{*}-e_{6}^{*} \odot e_{15}^{*}+e_{9}^{*} \odot e_{14}^{*}-e_{11}^{*} \odot e_{12}^{*}\right)+\lambda_{10}\left(e_{7}^{*} \odot e_{16}^{*}-e_{8}^{*} \odot e_{15}^{*}+e_{10}^{*} \odot e_{14}^{*}-e_{12}^{*} \odot e_{13}^{*}\right)
\end{aligned}
$$

for the generator $f$ of $\hat{\mathfrak{f}}(v)$. Then we calculate

$$
\begin{aligned}
f=\alpha_{1} \odot \alpha_{11}-\alpha_{2} \odot \alpha_{9}+\alpha_{3} \odot \alpha_{6}- & \alpha_{4} \odot \alpha_{5} \\
& +\lambda\left(e_{7}^{*} \odot e_{16}^{*}-e_{8}^{*} \odot e_{15}^{*}+e_{10}^{*} \odot e_{14}^{*}-e_{12}^{*} \odot e_{13}^{*}\right),
\end{aligned}
$$

where $\lambda=\lambda_{10}-\lambda_{2} \lambda_{9}+\lambda_{3} \lambda_{8}-\lambda_{4} \lambda_{7}+\lambda_{5} \lambda_{6}$ and

$$
\begin{aligned}
& \alpha_{1}=e_{11}^{*}+\lambda_{2} e_{13}^{*}+\lambda_{3} e_{14}^{*}+\lambda_{4} e_{15}^{*}+\lambda_{6} e_{16}^{*}, \quad \alpha_{2}=-e_{9}^{*}-\lambda_{2} e_{10}^{*}-\lambda_{3} e_{12}^{*}+\lambda_{5} e_{15}^{*}+\lambda_{7} e_{16}^{*}, \\
& \alpha_{3}=e_{6}^{*}+\lambda_{2} e_{8}^{*}-\lambda_{4} e_{12}^{*}-\lambda_{5} e_{14}^{*}+\lambda_{8} e_{16}^{*}, \quad \alpha_{4}=-e_{5}^{*}-\lambda_{2} e_{7}^{*}-\lambda_{6} e_{12}^{*}-\lambda_{7} e_{14}^{*}-\lambda_{8} e_{15}^{*}, \\
& \alpha_{5}=-e_{4}^{*}+\lambda_{3} e_{8}^{*}+\lambda_{4} e_{10}^{*}+\lambda_{5} e_{13}^{*}+\lambda_{9} e_{16}^{*}, \quad \alpha_{6}=e_{3}^{*}-\lambda_{3} e_{7}^{*}+\lambda_{6} e_{10}^{*}+\lambda_{7} e_{13}^{*}-\lambda_{9} e_{15}^{*}, \\
& \alpha_{9}=-e_{2}^{*}-\lambda_{4} e_{7}^{*}-\lambda_{6} e_{8}^{*}+\lambda_{8} e_{13}^{*}+\lambda_{9} e_{14}^{*}, \quad \alpha_{11}=e_{1}^{*}-\lambda_{5} e_{7}^{*}-\lambda_{7} e_{8}^{*}-\lambda_{8} e_{10}^{*}-\lambda_{9} e_{12}^{*},
\end{aligned}
$$

Thus $f$ is a non-degenarate quadratic form in $S^{2}\left(W^{*}\right)$, where $W=\left\langle\left\{e_{1}, \ldots, e_{16}\right\}\right\rangle$. Hence $\hat{\mathfrak{f}}(v)$ is of finite type (see Case (5) of $\S 3$ in [YY2]).

Now let us construct the model equation of second order from the coordinate description of the standard differential system $\left(M_{\mathfrak{m}}, D_{\mathfrak{m}}\right)$. We calculate

$$
\begin{aligned}
\varpi & =\varpi_{1}+\lambda_{2} \varpi_{2}+\cdots+\lambda_{10} \varpi_{10} \\
& =d y_{1}+\lambda_{2} d y_{2}+\cdots+\lambda_{10} d y_{10}-\hat{p}_{1} d x_{1}-\cdots-\hat{p}_{16} d x_{16} \\
& =d\left(y_{1}+\lambda_{2} y_{2}+\cdots+\lambda_{10} y_{10}\right)-y_{2} d \lambda_{2}-\cdots-y_{10} d \lambda_{10}-\hat{p}_{1} d x_{1}-\cdots-\hat{p}_{16} d x_{16}, \\
& =d z-\hat{p}_{1} d x_{1}-\cdots-\hat{p}_{16} d x_{16}-\hat{p}_{17} d x_{17}-\cdots-\hat{p}_{25} d x_{25},
\end{aligned}
$$

where we put

$$
\begin{array}{rlrl}
z & =y_{1}+\lambda_{2} y_{2}+\cdots+\lambda_{10} y_{10}, & \\
\hat{p}_{1}=p_{11}+\lambda_{2} p_{13}+\lambda_{3} p_{14}+\lambda_{4} p_{15}+\lambda_{6} p_{16}, & \hat{p}_{2}=-p_{9}-\lambda_{2} p_{10}-\lambda_{3} p_{12}+\lambda_{5} p_{15}+\lambda_{7} p_{16}, \\
\hat{p}_{3}=p_{6}+\lambda_{2} p_{8}-\lambda_{4} p_{12}-\lambda_{5} p_{14}+\lambda_{8} p_{16}, & \hat{p}_{4}=-p_{5}-\lambda_{2} p_{7}-\lambda_{6} p_{12}-\lambda_{7} p_{14}-\lambda_{8} p_{15}, \\
\hat{p}_{5}=-p_{4}+\lambda_{3} p_{8}+\lambda_{4} p_{10}+\lambda_{5} p_{13}+\lambda_{9} p_{16}, & \hat{p}_{6}=p_{3}-\lambda_{3} p_{7}+\lambda_{6} p_{10}+\lambda_{7} p_{13}-\lambda_{9} p_{15}, \\
\hat{p}_{7}=-\lambda_{2} p_{4}-\lambda_{3} p_{6}-\lambda_{4} p_{9}-\lambda_{5} p_{11}+\lambda_{10} p_{16}, & \hat{p}_{8}=\lambda_{2} p_{3}+\lambda_{3} p_{5}-\lambda_{6} p_{9}-\lambda_{7} p_{11}-\lambda_{10} p_{15}, \\
\hat{p}_{9}=-p_{2}-\lambda_{4} p_{7}-\lambda_{6} p_{8}+\lambda_{8} p_{13}+\lambda_{9} p_{14}, & \hat{p}_{10}=-\lambda_{2} p_{2}+\lambda_{4} p_{5}+\lambda_{6} p_{6}-\lambda_{8} p_{11}+\lambda_{10} p_{14}, \\
\hat{p}_{11}=p_{1}-\lambda_{5} p_{7}-\lambda_{7} p_{8}-\lambda_{8} p_{10}-\lambda_{9} p_{12}, & \hat{p}_{12}=-\lambda_{3} p_{2}-\lambda_{4} p_{3}-\lambda_{6} p_{4}-\lambda_{9} p_{11}-\lambda_{10} p_{13}, \\
\hat{p}_{13}=\lambda_{2} p_{1}+\lambda_{5} p_{5}+\lambda_{7} p_{6}+\lambda_{8} p_{9}-\lambda_{10} p_{12}, & \hat{p}_{14}=\lambda_{3} p_{1}-\lambda_{5} p_{3}-\lambda_{7} p_{4}+\lambda_{9} p_{9}+\lambda_{10} p_{10}, \\
\hat{p}_{15}=\lambda_{4} p_{1}+\lambda_{5} p_{2}-\lambda_{8} p_{4}-\lambda_{9} p_{6}-\lambda_{10} p_{8}, & \hat{p}_{16}=\lambda_{6} p_{1}+\lambda_{7} p_{2}+\lambda_{8} p_{3}+\lambda_{9} p_{5}+\lambda_{10} p_{7}, \\
\hat{p}_{17}=y_{2}, \ldots, \hat{p}_{\alpha+15}=y_{\alpha}, \ldots, \hat{p}_{25}=y_{10}, & x_{17}=\lambda_{2}, \ldots, x_{\alpha+15}=\lambda_{\alpha}, \ldots, x_{25}=\lambda_{10} .
\end{array}
$$

Then we have, for $\left(R\left(M_{\mathfrak{m}}\right) ; D_{M_{\mathfrak{m}}}^{1}, D_{M_{\mathfrak{m}}}^{2}\right)$,

$$
D_{M_{\mathfrak{m}}}^{1}=\{\varpi=0\}, \quad D_{M_{\mathfrak{m}}}^{2}=\left\{\varpi_{k}=\pi_{i}=0 \quad(1 \leqq k \leqq 10,1 \leqq i \leqq 16)\right\},
$$

where we denote the pullback on $R\left(M_{\mathfrak{m}}\right)$ of 1 -forms on $M_{\mathfrak{m}}$ by the same symbol. By taking the exterior derivatives of both sides of the above defining equations for $\hat{p}_{i}(i=1, \ldots, 16)$, we put

$$
\hat{\pi}_{1}=d \hat{p}_{1}-a\left(d x_{11}+x_{17} d x_{13}+x_{18} d x_{14}+x_{19} d x_{15}+x_{21} d x_{16}\right)
$$




$$
\begin{aligned}
& -p_{13} d x_{17}-p_{14} d x_{18}-p_{15} d x_{19}-p_{16} d x_{21}, \\
& \hat{\pi}_{2}=d \hat{p}_{2}-a\left(-d x_{9}-x_{17} d x_{10}-x_{18} d x_{12}+x_{20} d x_{15}+x_{22} d x_{16}\right) \\
& +p_{10} d x_{17}+p_{12} d x_{18}-p_{15} d x_{20}-p_{16} d x_{22}, \\
& \hat{\pi}_{3}=d \hat{p}_{3}-a\left(d x_{6}+x_{17} d x_{8}-x_{19} d x_{12}-x_{20} d x_{14}+x_{23} d x_{16}\right) \\
& -p_{8} d x_{17}+p_{12} d x_{19}+p_{14} d x_{20}-p_{16} d x_{23} \text {, } \\
& \hat{\pi}_{4}=d \hat{p}_{4}-a\left(-d x_{5}-x_{17} d x_{7}-x_{21} d x_{12}-x_{22} d x_{14}-x_{23} d x_{15}\right) \\
& +p_{7} d x_{17}+p_{12} d x_{21}+p_{14} d x_{22}+p_{15} d x_{23}, \\
& \hat{\pi}_{5}=d \hat{p}_{5}-a\left(-d x_{4}+x_{18} d x_{8}+x_{19} d x_{10}+x_{20} d x_{13}+x_{24} d x_{16}\right) \\
& -p_{8} d x_{18}-p_{10} d x_{19}-p_{13} d x_{20}-p_{16} d x_{24} \\
& \hat{\pi}_{6}=d \hat{p}_{6}-a\left(d x_{3}-x_{18} d x_{7}+x_{21} d x_{10}+x_{22} d x_{13}-x_{24} d x_{15}\right) \\
& +p_{7} d x_{18}-p_{10} d x_{21}-p_{13} d x_{22}+p_{15} d x_{24}, \\
& \hat{\pi}_{7}=d \hat{p}_{7}-a\left(-x_{17} d x_{4}-x_{18} d x_{6}-x_{19} d x_{9}-x_{20} d x_{11}+x_{25} d x_{16}\right) \\
& +p_{4} d x_{17}+p_{6} d x_{18}+p_{9} d x_{19}+p_{11} d x_{20}-p_{16} d x_{25}, \\
& \hat{\pi}_{8}=d \hat{p}_{8}-a\left(x_{17} d x_{3}+x_{18} d x_{5}-x_{21} d x_{9}-x_{22} d x_{11}-x_{25} d x_{15}\right) \\
& -p_{3} d x_{17}-p_{5} d x_{18}+p_{9} d x_{21}+p_{11} d x_{22}+p_{15} d x_{25} \text {, } \\
& \hat{\pi}_{9}=d \hat{p}_{9}-a\left(-d x_{2}-x_{19} d x_{7}-x_{21} d x_{8}+x_{23} d x_{13}+x_{24} d x_{14}\right) \\
& +p_{7} d x_{19}+p_{8} d x_{21}-p_{13} d x_{23}-p_{14} d x_{24}, \\
& \hat{\pi}_{10}=d \hat{p}_{10}-a\left(-x_{17} d x_{2}+x_{19} d x_{5}+x_{21} d x_{6}-x_{23} d x_{11}+x_{25} d x_{14}\right) \\
& +p_{2} d x_{17}-p_{5} d x_{19}-p_{6} d x_{21}+p_{11} d x_{23}-p_{14} d x_{25} \text {, } \\
& \hat{\pi}_{11}=d \hat{p}_{11}-a\left(d x_{1}-x_{20} d x_{7}-x_{22} d x_{8}-x_{23} d x_{10}-x_{24} d x_{12}\right) \\
& +p_{7} d x_{20}+p_{8} d x_{22}+p_{10} d x_{23}+p_{12} d x_{24}, \\
& \hat{\pi}_{12}=d \hat{p}_{12}-a\left(-x_{18} d x_{2}-x_{19} d x_{3}-x_{21} d x_{4}-x_{24} d x_{11}-x_{25} d x_{13}\right) \\
& +p_{2} d x_{18}+p_{3} d x_{19}+p_{4} d x_{21}+p_{11} d x_{24}+p_{13} d x_{25} \text {, } \\
& \hat{\pi}_{13}=d \hat{p}_{13}-a\left(x_{17} d x_{1}+x_{20} d x_{5}+x_{22} d x_{6}+x_{23} d x_{9}-x_{25} d x_{12}\right) \\
& -p_{1} d x_{17}-p_{5} d x_{20}-p_{6} d x_{22}-p_{9} d x_{23}+p_{12} d x_{25} \text {, } \\
& \hat{\pi}_{14}=d \hat{p}_{14}-a\left(x_{18} d x_{1}-x_{20} d x_{3}-x_{22} d x_{4}+x_{24} d x_{9}+x_{25} d x_{10}\right) \\
& -p_{1} d x_{18}+p_{3} d x_{20}+p_{4} d x_{22}-p_{9} d x_{24}-p_{10} d x_{25} \text {, } \\
& \hat{\pi}_{15}=d \hat{p}_{15}-a\left(x_{19} d x_{1}+x_{20} d x_{2}-x_{23} d x_{4}-x_{24} d x_{6}-x_{25} d x_{8}\right) \\
& -p_{1} d x_{19}-p_{2} d x_{20}+p_{4} d x_{23}+p_{6} d x_{24}+p_{8} d x_{25} \text {, } \\
& \hat{\pi}_{16}=d \hat{p}_{16}-a\left(x_{21} d x_{1}+x_{22} d x_{2}+x_{23} d x_{3}+x_{24} d x_{5}+x_{25} d x_{7}\right) \\
& -p_{1} d x_{21}-p_{2} d x_{22}-p_{3} d x_{23}-p_{5} d x_{24}-p_{7} d x_{25} \text {. }
\end{aligned}
$$

Then we see that $\left\{\hat{\pi}_{1}, \ldots, \hat{\pi}_{16}\right\}$ can be written as the linear combinations of $\left\{\pi_{1}, \ldots, \pi_{16}\right\}$ with the same coefficients (in $\lambda$ 's) such that $\left\{\hat{p}_{1}, \ldots, \hat{p}_{16}\right\}$ are written as the liear combination of $\left\{p_{1}, \ldots, p_{16}\right\}$ as in the above equations. Hence we have

$$
D_{M_{\mathfrak{m}}}^{1}=\{\varpi=o\}, \quad D_{M_{\mathfrak{m}}}^{2}=\left\{\varpi=\hat{\pi}_{1}=\cdots=\hat{\pi}_{16}=\hat{\pi}_{17}=\cdots=\hat{\pi}_{25}=0\right\},
$$

where $\hat{\pi}_{15+\alpha}=\varpi_{\alpha}(2 \leqq \alpha \leqq 10)$ are written as follows:

$\hat{\pi}_{17}=d \hat{p}_{17}-p_{13} d x_{1}+p_{10} d x_{2}-p_{8} d x_{3}+p_{7} d x_{4}+p_{4} d x_{7}-p_{3} d x_{8}+p_{2} d x_{10}-p_{1} d x_{13}$, 


$$
\begin{aligned}
& \hat{\pi}_{18}=d \hat{p}_{18}-p_{14} d x_{1}+p_{12} d x_{2}-p_{8} d x_{5}+p_{7} d x_{6}+p_{6} d x_{7}-p_{5} d x_{8}+p_{2} d x_{12}-p_{1} d x_{14}, \\
& \hat{\pi}_{19}=d \hat{p}_{19}-p_{15} d x_{1}+p_{12} d x_{3}-p_{10} d x_{5}+p_{9} d x_{7}+p_{7} d x_{9}-p_{5} d x_{10}+p_{3} d x_{12}-p_{1} d x_{15}, \\
& \hat{\pi}_{20}=d \hat{p}_{20}-p_{15} d x_{2}+p_{14} d x_{3}-p_{13} d x_{5}+p_{11} d x_{7}+p_{7} d x_{11}-p_{5} d x_{13}+p_{3} d x_{14}-p_{2} d x_{15}, \\
& \hat{\pi}_{21}=d \hat{p}_{21}-p_{16} d x_{1}+p_{12} d x_{4}-p_{10} d x_{6}+p_{9} d x_{8}+p_{8} d x_{9}-p_{6} d x_{10}+p_{4} d x_{12}-p_{1} d x_{16}, \\
& \hat{\pi}_{22}=d \hat{p}_{22}-p_{16} d x_{2}+p_{14} d x_{4}-p_{13} d x_{6}+p_{11} d x_{8}+p_{8} d x_{11}-p_{6} d x_{13}+p_{4} d x_{14}-p_{2} d x_{16}, \\
& \hat{\pi}_{23}=d \hat{p}_{23}-p_{16} d x_{3}+p_{15} d x_{4}-p_{13} d x_{9}+p_{11} d x_{10}+p_{10} d x_{11}-p_{9} d x_{13}+p_{4} d x_{15}-p_{3} d x_{16}, \\
& \hat{\pi}_{24}=d \hat{p}_{24}-p_{16} d x_{5}+p_{15} d x_{6}-p_{14} d x_{9}+p_{12} d x_{11}+p_{11} d x_{12}-p_{9} d x_{14}+p_{6} d x_{15}-p_{5} d x_{16}, \\
& \hat{\pi}_{25}=d \hat{p}_{25}-p_{16} d x_{7}+p_{15} d x_{8}-p_{14} d x_{10}+p_{13} d x_{12}+p_{12} d x_{13}-p_{10} d x_{14}+p_{8} d x_{15}-p_{7} d x_{16} .
\end{aligned}
$$

Moreover we calculate

$$
\begin{aligned}
\lambda p_{16} & =x_{20} \hat{p}_{1}-x_{19} \hat{p}_{2}+x_{18} \hat{p}_{3}-x_{17} \hat{p}_{5}+\hat{p}_{7}, \\
\lambda p_{15} & =-x_{22} \hat{p}_{1}+x_{21} \hat{p}_{2}-x_{18} \hat{p}_{4}+x_{17} \hat{p}_{6}-\hat{p}_{8}, \\
\lambda p_{14} & =x_{23} \hat{p}_{1}-x_{21} \hat{p}_{3}+x_{19} \hat{p}_{4}-x_{17} \hat{p}_{9}+\hat{p}_{10}, \\
\lambda p_{13} & =-x_{24} \hat{p}_{1}+x_{21} \hat{p}_{5}-x_{19} \hat{p}_{6}+x_{18} \hat{p}_{9}-\hat{p}_{12}, \\
\lambda p_{12} & =-x_{23} \hat{p}_{2}+x_{22} \hat{p}_{3}-x_{20} \hat{p}_{4}+x_{17} \hat{p}_{11}-\hat{p}_{13}, \\
\lambda p_{11} & =x_{25} \hat{p}_{1}-x_{21} \hat{p}_{7}+x_{19} \hat{p}_{8}-x_{18} \hat{p}_{10}+x_{17} \hat{p}_{12}, \\
\lambda p_{10} & =x_{24} \hat{p}_{2}-x_{22} \hat{p}_{5}+x_{20} \hat{p}_{6}-x_{18} \hat{p}_{11}+\hat{p}_{14}, \\
\lambda p_{9} & =-x_{25} \hat{p}_{2}+x_{22} \hat{p}_{7}-x_{20} \hat{p}_{8}+x_{18} \hat{p}_{13}-x_{17} \hat{p}_{14}, \\
\lambda p_{8} & =-x_{24} \hat{p}_{3}+x_{23} \hat{p}_{5}-x_{20} \hat{p}_{9}+x_{19} \hat{p}_{11}-\hat{p}_{15}, \\
\lambda p_{7} & =x_{24} \hat{p}_{4}-x_{23} \hat{p}_{6}+x_{22} \hat{p}_{9}-x_{21} \hat{p}_{11}+\hat{p}_{16}, \\
\lambda p_{6} & =x_{25} \hat{p}_{3}-x_{23} \hat{p}_{7}+x_{20} \hat{p}_{10}-x_{19} \hat{p}_{13}+x_{17} \hat{p}_{15}, \\
\lambda p_{5} & =-x_{25} \hat{p}_{4}+x_{23} \hat{p}_{8}-x_{22} \hat{p}_{10}+x_{21} \hat{p}_{13}-x_{17} \hat{p}_{16}, \\
\lambda p_{4} & =-x_{25} \hat{p}_{5}+x_{24} \hat{p}_{7}-x_{20} \hat{p}_{12}+x_{19} \hat{p}_{14}-x_{18} \hat{p}_{15}, \\
\lambda p_{3} & =x_{25} \hat{p}_{6}-x_{24} \hat{p}_{8}+x_{22} \hat{p}_{12}-x_{21} \hat{p}_{14}+x_{18} \hat{p}_{16}, \\
\lambda p_{2} & =-x_{25} \hat{p}_{9}+x_{24} \hat{p}_{10}-x_{23} \hat{p}_{12}+x_{21} \hat{p}_{15}-x_{19} \hat{p}_{16}, \\
\lambda p_{1} & =x_{25} \hat{p}_{11}-x_{24} \hat{p}_{13}+x_{23} \hat{p}_{14}-x_{22} \hat{p}_{15}+x_{20} \hat{p}_{16},
\end{aligned}
$$

where $\lambda=x_{25}-x_{17} x_{24}+x_{18} x_{23}-x_{19} x_{22}+x_{20} x_{21}$. Thus we obtain the following model equation of second order :

$$
\begin{aligned}
& \frac{\partial^{2} z}{\partial x_{1} \partial x_{11}}=-\frac{\partial^{2} z}{\partial x_{2} \partial x_{9}}=\frac{\partial^{2} z}{\partial x_{3} \partial x_{6}}=-\frac{\partial^{2} z}{\partial x_{4} \partial x_{5}} \quad(=a) \\
& \frac{\partial^{2} z}{\partial x_{1} \partial x_{13}}=-\frac{\partial^{2} z}{\partial x_{2} \partial x_{10}}=\frac{\partial^{2} z}{\partial x_{3} \partial x_{8}}=-\frac{\partial^{2} z}{\partial x_{4} \partial x_{7}}=x_{17} \frac{\partial^{2} z}{\partial x_{1} \partial x_{11}} \\
& \frac{\partial^{2} z}{\partial x_{1} \partial x_{14}}=-\frac{\partial^{2} z}{\partial x_{2} \partial x_{12}}=\frac{\partial^{2} z}{\partial x_{5} \partial x_{8}}=-\frac{\partial^{2} z}{\partial x_{6} \partial x_{7}}=x_{18} \frac{\partial^{2} z}{\partial x_{1} \partial x_{11}} \\
& \frac{\partial^{2} z}{\partial x_{1} \partial x_{15}}=-\frac{\partial^{2} z}{\partial x_{3} \partial x_{12}}=\frac{\partial^{2} z}{\partial x_{5} \partial x_{10}}=-\frac{\partial^{2} z}{\partial x_{7} \partial x_{9}}=x_{19} \frac{\partial^{2} z}{\partial x_{1} \partial x_{11}} \\
& \frac{\partial^{2} z}{\partial x_{2} \partial x_{15}}=-\frac{\partial^{2} z}{\partial x_{3} \partial x_{14}}=\frac{\partial^{2} z}{\partial x_{5} \partial x_{13}}=-\frac{\partial^{2} z}{\partial x_{7} \partial x_{11}}=x_{20} \frac{\partial^{2} z}{\partial x_{1} \partial x_{11}}
\end{aligned}
$$




$$
\begin{aligned}
& \frac{\partial^{2} z}{\partial x_{1} \partial x_{16}}=-\frac{\partial^{2} z}{\partial x_{4} \partial x_{12}}=\frac{\partial^{2} z}{\partial x_{6} \partial x_{10}}=-\frac{\partial^{2} z}{\partial x_{8} \partial x_{9}}=x_{21} \frac{\partial^{2} z}{\partial x_{1} \partial x_{11}} \\
& \frac{\partial^{2} z}{\partial x_{2} \partial x_{16}}=-\frac{\partial^{2} z}{\partial x_{4} \partial x_{14}}=\frac{\partial^{2} z}{\partial x_{6} \partial x_{13}}=-\frac{\partial^{2} z}{\partial x_{8} \partial x_{11}}=x_{22} \frac{\partial^{2} z}{\partial x_{1} \partial x_{11}} \\
& \frac{\partial^{2} z}{\partial x_{3} \partial x_{16}}=-\frac{\partial^{2} z}{\partial x_{4} \partial x_{15}}=\frac{\partial^{2} z}{\partial x_{9} \partial x_{13}}=-\frac{\partial^{2} z}{\partial x_{10} \partial x_{11}}=x_{23} \frac{\partial^{2} z}{\partial x_{1} \partial x_{11}} \\
& \frac{\partial^{2} z}{\partial x_{5} \partial x_{16}}=-\frac{\partial^{2} z}{\partial x_{6} \partial x_{15}}=\frac{\partial^{2} z}{\partial x_{9} \partial x_{14}}=-\frac{\partial^{2} z}{\partial x_{11} \partial x_{12}}=x_{24} \frac{\partial^{2} z}{\partial x_{1} \partial x_{11}} \\
& \frac{\partial^{2} z}{\partial x_{7} \partial x_{16}}=-\frac{\partial^{2} z}{\partial x_{8} \partial x_{15}}=\frac{\partial^{2} z}{\partial x_{10} \partial x_{14}}=-\frac{\partial^{2} z}{\partial x_{12} \partial x_{13}}=x_{25} \frac{\partial^{2} z}{\partial x_{1} \partial x_{11}} \\
& \frac{\partial^{2} z}{\partial x_{13} \partial x_{17}}=\frac{\partial^{2} z}{\partial x_{14} \partial x_{18}}=\frac{\partial^{2} z}{\partial x_{15} \partial x_{19}}=\frac{\partial^{2} z}{\partial x_{16} \partial x_{21}} \quad\left(=p_{1}\right) \\
& =\lambda^{-1}\left(x_{25} \frac{\partial z}{\partial x_{11}}-x_{24} \frac{\partial z}{\partial x_{13}}+x_{23} \frac{\partial z}{\partial x_{14}}-x_{22} \frac{\partial z}{\partial x_{15}}+x_{20} \frac{\partial z}{\partial x_{16}}\right), \\
& \frac{\partial^{2} z}{\partial x_{10} \partial x_{17}}=\frac{\partial^{2} z}{\partial x_{12} \partial x_{18}}=-\frac{\partial^{2} z}{\partial x_{15} \partial x_{20}}=-\frac{\partial^{2} z}{\partial x_{16} \partial x_{22}} \quad\left(=-p_{2}\right) \\
& =\lambda^{-1}\left(x_{25} \frac{\partial z}{\partial x_{9}}-x_{24} \frac{\partial z}{\partial x_{10}}+x_{23} \frac{\partial z}{\partial x_{12}}-x_{21} \frac{\partial z}{\partial x_{15}}+x_{19} \frac{\partial z}{\partial x_{16}}\right), \\
& \frac{\partial^{2} z}{\partial x_{8} \partial x_{17}}=-\frac{\partial^{2} z}{\partial x_{12} \partial x_{19}}=-\frac{\partial^{2} z}{\partial x_{14} \partial x_{20}}=\frac{\partial^{2} z}{\partial x_{16} \partial x_{23}} \quad\left(=p_{3}\right) \\
& =\lambda^{-1}\left(x_{25} \frac{\partial z}{\partial x_{6}}-x_{24} \frac{\partial z}{\partial x_{8}}+x_{22} \frac{\partial z}{\partial x_{12}}-x_{21} \frac{\partial z}{\partial x_{14}}+x_{18} \frac{\partial z}{\partial x_{16}}\right), \\
& \frac{\partial^{2} z}{\partial x_{7} \partial x_{17}}=\frac{\partial^{2} z}{\partial x_{12} \partial x_{21}}=\frac{\partial^{2} z}{\partial x_{14} \partial x_{22}}=\frac{\partial^{2} z}{\partial x_{15} \partial x_{23}} \quad\left(=-p_{4}\right) \\
& =\lambda^{-1}\left(x_{25} \frac{\partial z}{\partial x_{5}}-x_{24} \frac{\partial z}{\partial x_{7}}+x_{20} \frac{\partial z}{\partial x_{12}}-x_{19} \frac{\partial z}{\partial x_{14}}+x_{18} \frac{\partial z}{\partial x_{15}}\right), \\
& \frac{\partial^{2} z}{\partial x_{8} \partial x_{18}}=\frac{\partial^{2} z}{\partial x_{10} \partial x_{19}}=\frac{\partial^{2} z}{\partial x_{13} \partial x_{20}}=\frac{\partial^{2} z}{\partial x_{16} \partial x_{24}} \quad\left(=p_{5}\right) \\
& =\lambda^{-1}\left(-x_{25} \frac{\partial z}{\partial x_{4}}+x_{23} \frac{\partial z}{\partial x_{8}}-x_{22} \frac{\partial z}{\partial x_{10}}+x_{21} \frac{\partial z}{\partial x_{13}}-x_{17} \frac{\partial z}{\partial x_{16}}\right), \\
& -\frac{\partial^{2} z}{\partial x_{7} \partial x_{18}}=\frac{\partial^{2} z}{\partial x_{10} \partial x_{21}}=\frac{\partial^{2} z}{\partial x_{13} \partial x_{22}}=-\frac{\partial^{2} z}{\partial x_{15} \partial x_{24}} \quad\left(=p_{6}\right) \\
& =\lambda^{-1}\left(x_{25} \frac{\partial z}{\partial x_{3}}-x_{23} \frac{\partial z}{\partial x_{7}}+x_{20} \frac{\partial z}{\partial x_{10}}-x_{19} \frac{\partial z}{\partial x_{13}}+x_{17} \frac{\partial z}{\partial x_{15}}\right), \\
& -\frac{\partial^{2} z}{\partial x_{4} \partial x_{17}}=-\frac{\partial^{2} z}{\partial x_{6} \partial x_{18}}=-\frac{\partial^{2} z}{\partial x_{9} \partial x_{19}}=-\frac{\partial^{2} z}{\partial x_{11} \partial x_{20}}=\frac{\partial^{2} z}{\partial x_{16} \partial x_{25}} \quad\left(=p_{7}\right) \\
& =\lambda^{-1}\left(x_{24} \frac{\partial z}{\partial x_{4}}-x_{23} \frac{\partial z}{\partial x_{6}}+x_{22} \frac{\partial z}{\partial x_{9}}-x_{21} \frac{\partial z}{\partial x_{11}}+\frac{\partial z}{\partial x_{16}}\right), \\
& -\frac{\partial^{2} z}{\partial x_{3} \partial x_{17}}=-\frac{\partial^{2} z}{\partial x_{5} \partial x_{18}}=\frac{\partial^{2} z}{\partial x_{9} \partial x_{21}}=\frac{\partial^{2} z}{\partial x_{11} \partial x_{22}}=\frac{\partial^{2} z}{\partial x_{15} \partial x_{25}} \quad\left(=-p_{8}\right) \\
& =\lambda^{-1}\left(x_{24} \frac{\partial z}{\partial x_{3}}-x_{23} \frac{\partial z}{\partial x_{5}}+x_{20} \frac{\partial z}{\partial x_{9}}-x_{19} \frac{\partial z}{\partial x_{11}}+\frac{\partial z}{\partial x_{15}}\right),
\end{aligned}
$$




$$
\begin{aligned}
& \frac{\partial^{2} z}{\partial x_{7} \partial x_{19}}=\frac{\partial^{2} z}{\partial x_{8} \partial x_{21}}=-\frac{\partial^{2} z}{\partial x_{13} \partial x_{23}}=-\frac{\partial^{2} z}{\partial x_{14} \partial x_{24}} \quad\left(=-p_{9}\right) \\
& =\lambda^{-1}\left(x_{25} \frac{\partial z}{\partial x_{2}}-x_{22} \frac{\partial z}{\partial x_{7}}+x_{20} \frac{\partial z}{\partial x_{8}}-x_{18} \frac{\partial z}{\partial x_{13}}+x_{17} \frac{\partial z}{\partial x_{14}}\right), \\
& -\frac{\partial^{2} z}{\partial x_{2} \partial x_{17}}=\frac{\partial^{2} z}{\partial x_{5} \partial x_{19}}=\frac{\partial^{2} z}{\partial x_{6} \partial x_{21}}=-\frac{\partial^{2} z}{\partial x_{11} \partial x_{23}}=\frac{\partial^{2} z}{\partial x_{14} \partial x_{25}} \quad\left(=p_{10}\right) \\
& =\lambda^{-1}\left(x_{24} \frac{\partial z}{\partial x_{2}}-x_{22} \frac{\partial z}{\partial x_{5}}+x_{20} \frac{\partial z}{\partial x_{6}}-x_{18} \frac{\partial z}{\partial x_{11}}+\frac{\partial z}{\partial x_{14}}\right), \\
& -\frac{\partial^{2} z}{\partial x_{7} \partial x_{20}}=-\frac{\partial^{2} z}{\partial x_{8} \partial x_{22}}=-\frac{\partial^{2} z}{\partial x_{10} \partial x_{23}}=-\frac{\partial^{2} z}{\partial x_{12} \partial x_{24}} \quad\left(=p_{11}\right) \\
& =\lambda^{-1}\left(x_{25} \frac{\partial z}{\partial x_{1}}-x_{21} \frac{\partial z}{\partial x_{7}}+x_{19} \frac{\partial z}{\partial x_{8}}-x_{18} \frac{\partial z}{\partial x_{10}}+x_{17} \frac{\partial z}{\partial x_{12}}\right), \\
& \frac{\partial^{2} z}{\partial x_{2} \partial x_{18}}=\frac{\partial^{2} z}{\partial x_{3} \partial x_{19}}=\frac{\partial^{2} z}{\partial x_{4} \partial x_{21}}=\frac{\partial^{2} z}{\partial x_{11} \partial x_{24}}=\frac{\partial^{2} z}{\partial x_{13} \partial x_{25}} \quad\left(=-p_{12}\right) \\
& =\lambda^{-1}\left(x_{23} \frac{\partial z}{\partial x_{2}}-x_{22} \frac{\partial z}{\partial x_{3}}+x_{20} \frac{\partial z}{\partial x_{4}}-x_{17} \frac{\partial z}{\partial x_{11}}+\frac{\partial z}{\partial x_{13}}\right), \\
& -\frac{\partial^{2} z}{\partial x_{1} \partial x_{17}}=-\frac{\partial^{2} z}{\partial x_{5} \partial x_{20}}=-\frac{\partial^{2} z}{\partial x_{6} \partial x_{22}}=-\frac{\partial^{2} z}{\partial x_{9} \partial x_{23}}=\frac{\partial^{2} z}{\partial x_{12} \partial x_{25}} \quad\left(=-p_{13}\right) \\
& =\lambda^{-1}\left(x_{24} \frac{\partial z}{\partial x_{1}}-x_{21} \frac{\partial z}{\partial x_{5}}+x_{19} \frac{\partial z}{\partial x_{6}}-x_{18} \frac{\partial z}{\partial x_{9}}+\frac{\partial z}{\partial x_{12}}\right), \\
& \frac{\partial^{2} z}{\partial x_{1} \partial x_{18}}=-\frac{\partial^{2} z}{\partial x_{3} \partial x_{20}}=-\frac{\partial^{2} z}{\partial x_{4} \partial x_{22}}=\frac{\partial^{2} z}{\partial x_{9} \partial x_{24}}=\frac{\partial^{2} z}{\partial x_{10} \partial x_{25}} \quad\left(=p_{14}\right) \\
& =\lambda^{-1}\left(x_{23} \frac{\partial z}{\partial x_{1}}-x_{21} \frac{\partial z}{\partial x_{3}}+x_{19} \frac{\partial z}{\partial x_{4}}-x_{17} \frac{\partial z}{\partial x_{9}}+\frac{\partial z}{\partial x_{10}}\right), \\
& -\frac{\partial^{2} z}{\partial x_{1} \partial x_{19}}=-\frac{\partial^{2} z}{\partial x_{2} \partial x_{20}}=\frac{\partial^{2} z}{\partial x_{4} \partial x_{23}}=\frac{\partial^{2} z}{\partial x_{6} \partial x_{24}}=\frac{\partial^{2} z}{\partial x_{8} \partial x_{25}} \quad\left(=-p_{15}\right) \\
& =\lambda^{-1}\left(x_{22} \frac{\partial z}{\partial x_{1}}-x_{21} \frac{\partial z}{\partial x_{2}}+x_{18} \frac{\partial z}{\partial x_{4}}-x_{17} \frac{\partial z}{\partial x_{6}}+\frac{\partial z}{\partial x_{8}}\right), \\
& \frac{\partial^{2} z}{\partial x_{1} \partial x_{21}}=\frac{\partial^{2} z}{\partial x_{2} \partial x_{22}}=\frac{\partial^{2} z}{\partial x_{3} \partial x_{23}}=\frac{\partial^{2} z}{\partial x_{5} \partial x_{24}}=\frac{\partial^{2} z}{\partial x_{7} \partial x_{25}} \quad\left(=p_{16}\right) \\
& =\lambda^{-1}\left(x_{20} \frac{\partial z}{\partial x_{1}}-x_{19} \frac{\partial z}{\partial x_{2}}+x_{18} \frac{\partial z}{\partial x_{3}}-x_{17} \frac{\partial z}{\partial x_{5}}+\frac{\partial z}{\partial x_{7}}\right), \\
& \frac{\partial^{2} z}{\partial x_{i} \partial x_{j}}=0 \quad \text { otherwise. }
\end{aligned}
$$

One can check that, among simple graded Lie algebras (of depth 3 ) of class $(D)$ in $\S 5$ of [YY2], regular differential systems $(X, D)$ of type $\mathfrak{m}$ satisfy the condition $(X .1)$ to $(X .3)$ in $\S 4.3$ when $\mathfrak{m}$ is the negative part of one of the simple graded Lie algebras $\left(C_{\ell},\left\{\alpha_{i}, \alpha_{\ell}\right\}\right)$ for $i=2, \ldots, \ell-1,\left(D_{\ell},\left\{\alpha_{i}, \alpha_{\ell}\right\}\right)(2<i<\ell-1)$, when $i$ is even, or $\left(E_{7},\left\{\alpha_{6} . \alpha_{7}\right\}\right)$, whereas the condition $(X .3)$ is not satisfied by the other cases. 


\section{REFERENCES}

[Bai] T.N.Baily, Parabolic Invariant Theory and Geometry in "The Penrose Transform and Analytic Cohomology in Representation Theory" Contemp. Math. 154, Amer. Math. Soc., (1993).

[Bo] W. M. Boothby, Homogeneous complex contact manifolds, Proc. Symp. Pure Math., Amer. Math. Soc. 3 (1961), 144-154.

[Bu] N.Bourbaki, Groupes et algèbles de Lie, Chapitre 4,5 et 6, Hermann, Paris (1968).

$\left[\mathrm{BCG}_{3}\right]$ R. Bryant, S. S. Chern, R. B. Gardner, H. Goldschmidt, and P. Griffiths, Exterior differential systems, Springer-Verlag, New-York (1986).

[C1] E.Cartan, Les systèmes de Pfaff à cinq variables et les équations aux dérivées partielles du second ordre, Ann. Ec. Normale 27 (1910), 109-192.

[C2] -----, Sur les systèmes en involution d'équations aux dérivées partielles du second ordre à une fonction inconnue de trois variables indépendantes, Bull. Soc. Math. France 39 (1911), 352-443.

[HY] J.Hwang and K. Yamaguchi, Characterization of hermitian Symmetric Spaces by Fundamental Forms, Duke Math. J. 120 No.3 (2003), 621-634.

[Hu] J. E. Humphreys, Introduction to Lie Algebras and Representation Theory, Springer-Verlag, New York (1972).

[Ko] B. Kostant, Lie algebra cohomology and generalized Borel-Weil theorem, Ann. Math. 74 (1961), 329-387.

[Ku] M. Kuranishi, Lectures on involutive systems of partial differential equations, Pub.Soc.Mat., São Paulo (1967).

[SYY] T. Sasaki, K. Yamaguchi and M. Yoshida, On the Rigidity of Differential Systems modeled on Hermitian Symmetric Spaces and Disproofs of a Conjecture concerning Modular Interpretations of Configuration Spaces, Advanced Studies in Pure Math. 25 (1997), 318-354.

[Se] Y. Se-ashi, On differential invariants of integrable finite type linear differential equations, Hokkaido Math.J. 17 (1988), 151-195.

[St] S. Sternberg Lectures on Differential Geometry, Prentice-Hall, New Jersey (1964).

[T1] N. Tanaka On generalized graded Lie algebras and geometric structures I, J. Math. Soc. Japan 19 (1967), 215-254.

[T2] _(1970), 1-82.

[T3] _-_, On non-degenerate real hypersurfaces, graded Lie algebras and Cartan connections, Japan. J. Math.2 (1976), 131-190.

[T4] -_-- On the equivalence problems associated with simple graded Lie algebras, Hokkaido Math. J. 8 (1979), 23-84.

[T5] _-_--, On geometry and integration of systems of second order ordinary differential equations, Proc. Symposium on Differential Geometry, 1982, 194-205 (in Japanese)

[Y1] K.Yamaguchi Contact geometry of higher order, Japanese J. of Math. 8 (1982), 109-176.

[Y2] -----, On involutive systems of second order of codimension 2, Proc. of Japan Acad. 58, Ser A, No.7 (1982), 302-305.

[Y3] -----, Geometrization of Jet bundles, Hokkaido Math. J. 12 (1983), 27-40.

[Y4] -_--, Typical classes in involutive systems of second order, Japanese J. Math. 11 (1985), 265-291.

[Y5] ----, Differential systems associated with simple graded Lie algebras, Adv. Studies in Pure Math. 22 (1993), 413-494.

[Y6] -----, $G_{2}$-Geometry of Overdetermined Systems of Second Order, Trends in Math. (Analysis and Geometry in Several Complex Variables) (1999), Birkhäuser, Boston 289-314.

[Y7] -----, Geometry of Linear Differential Systems Towards Contact Geometry of Second Order, IMA Volumes in Mathematics and its Applications 144 (Symmetries and Overdetermined Systems of Partial Differential Equations) (2007), 151-203.

[YY1] K. Yamaguchi and T. Yatsui, Geometry of Higher Order Differential Equations of Finite Type associated with Symmetric Spaces, Advanced Studies in Pure Mathematics 37 (2002), 397-458.

[YY2] K.Yamaguchi and T.Yatsui, Parabolic Geometries associated with Differential Equations of Finite Type, Progress in Mathematics 252 (From Geometry to Quantum Mechanics: In Honor of Hideki Omori) (2007), 161-209

K.Yamaguchi, Department of Mathematics,, Faculty of Science,, Hokkaido University,, SAPPORO 060-0810,, JAPAN, E-MAIL yamaguch@math.sci.hokudai.ac.jp 